\title{
Direct Search for Heavy Neutral Gauge Bosons in the Dielectron Channel at D0
}

\author{
Ioannis Katsanos
}

Submitted in partial fulfillment of the

requirements for the degree

of Doctor of Philosophy

in the Graduate School of Arts and Sciences

COLUMBIA UNIVERSITY

2008 
(C) 2008

Ioannis Katsanos

All Rights Reserved 


\title{
ABSTRACT
}

\section{Direct Search for Heavy Neutral Gauge Bosons in the Dielectron Channel at D0}

\author{
Ioannis Katsanos
}

The existence of a heavy partner of the $Z$ boson, a so-called $Z^{\prime}$ boson, is proposed in many extensions of the Standard Model, including grand unified theories, extended tecnhicolor models, and models with extra dimensions. This dissertation describes a direct search in the di-electron invariant mass spectrum for evidence of $Z^{\prime}$ production. The analysis used $1.106 \pm 0.067 \mathrm{fb}^{-1}$ of data collected from 2002 to 2006 with the D0 detector, which studies $p \bar{p}$ interactions at a center-of-mass energy of $\sqrt{\mathrm{s}}=1.96 \mathrm{TeV}$.

In the absence of a $Z^{\prime}$ signal, a $95 \%$ upper limit on the production cross section is set for a $Z^{\prime}$ decaying into dielectrons. The existence of a $Z^{\prime}$ with mass less than $920 \mathrm{GeV}$ is excluded at the $95 \%$ confidence level, assuming the sequential $\mathrm{Z}^{\prime}$ model. This result represents a significant improvement over the most stringent published limit from a direct search to date, namely $850 \mathrm{GeV}$. 


\section{Contents}

List of Tables $\quad$ V

List of Figures $\quad$ vii

Acknowledgements xii

1 Introduction 1

2 The Standard Model 3

2.1 The Standard Model . . . . . . . . . . . . . . . . . . . . . . 3

2.1 .1 Fermions . . . . . . . . . . . . . . . . 4

2.1.2 Gauge Bosons . . . . . . . . . . . . . . . . 6

2.1.3 Quantum Electrodynamics (QED) .......... 7

2.1.4 Electroweak .................. 8

2.1.5 Quantum Chromodynamics (QCD) ........... 10

2.1.6 Limitations of the Standard Model . . . . . . . . . . . . . . . 11 
$3 Z^{\prime}$ Models and Phenomenology 14

$3.1 \quad Z^{\prime}$ Models . . . . . . . . . . . . . . . . . . . 15

3.1.1 Grand Unification Theories . . . . . . . . . . . . . . 15

3.1.2 Left - Right Symmetric Model (LRM) . . . . . . . . . . . . 16

3.1.3 Superstring Theory . . . . . . . . . . . . . . . . . . . . . 17

3.1.4 "Sequential" Standard Model . . . . . . . . . . . . . . . 17

3.2 Previous Z' Searches . . . . . . . . . . . . . . . . . . 18

4 Experimental Apparatus $\quad 20$

4.1 Accelerator Chain . . . . . . . . . . . . . . . 20

4.2 The Do Detector . . . . . . . . . . . . . . . . . 23

4.2.1 Coordinate System . . . . . . . . . . . . . 25

4.2.2 Central Tracking . . . . . . . . . . . . . . . . . 26

4.2.3 Preshower Detectors . . . . . . . . . . . . . . . . 32

4.2.4 Calorimeters . . . . . . . . . . . . . . . . 36

4.2.5 Muon System . . . . . . . . . . . . . . . . . . . . 44

4.2.6 Luminosity Monitor . . . . . . . . . . . . . . . . . 47

4.3 The D0 Trigger and Data Acquisition Systems . . . . . . . . . . . . 48

4.3.1 Level 1 Trigger . . . . . . . . . . . . . . . 50

4.3 .2 Level 2 Trigger . . . . . . . . . . . . . . . . . . 53

4.3.3 Level 3 Trigger and Data Acquisition . . . . . . . . . . . . 56 
5 Offline Event Reconstruction and Object Identification

5.1 Tracking . . . . . . . . . . . . . . . . . . . . 59

5.2 Primary Vertices $\ldots \ldots \ldots \ldots \ldots \ldots$

5.3 Calorimeter Preprocessing . . . . . . . . . . . . . . . . . . 64

5.4 Electrons. . . . . . . . . . . . . . . . . . . . 65

5.5 Muons . . . . . . . . . . . . . . . . . . . . . 73

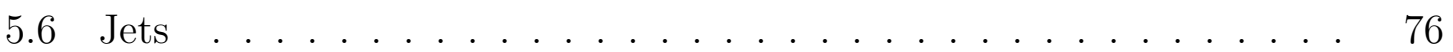

5.7 Missing Transverse Energy . . . . . . . . . . . . . . . . . 77

$\begin{array}{lll}6 & \text { Analysis } & 78\end{array}$

6.1 Data Selection . . . . . . . . . . . . . . . . . . 78

6.2 Backgrounds . . . . . . . . . . . . . . . . 80

6.2.1 Physics Backgrounds . . . . . . . . . . . . . . . . 80

6.2.2 Instrumental Background - Misidentified Electrons _. . . . . 84

6.3 Data Treatment . . . . . . . . . . . . . . . . . . . . 85

6.3.1 Correction for Non-fiducial Electrons _ . . . . . . . . . . 90

6.3.2 Correction for End-Cap Electrons . . . . . . . . . . . . 95

6.4 Background Normalization . . . . . . . . . . . . . . . . . 95

$6.4 .1 \quad \mathrm{CCCC} \ldots \ldots \ldots \ldots \ldots \ldots \ldots \ldots$

$6.4 .2 \mathrm{ECCC} \ldots \ldots \ldots \ldots \ldots \ldots \ldots \ldots$

6.5 Z' Signal Monte Carlo Studies . . . . . . . . . . . . . . . . 100

6.6 Extrapolating to Higher Invariant Masses . . . . . . . . . . . 108 iii 


\section{Extraction of Limit}

7.1 Introduction . . . . . . . . . . . . . . . . . . 113

7.2 Bayesian Technique . . . . . . . . . . . . . . . . . . . 114

7.3 Inputs to Limit Calculator . . . . . . . . . . . . . . . . . . . 116

7.3.1 Systematic Uncertainties . . . . . . . . . . . . . . . . . 117

7.3.2 Luminosity . . . . . . . . . . . . . . . . . . . 122

7.3.3 Data and Background Events . . . . . . . . . . . . . 122

7.3.4 Acceptance ..................... 123

7.4 Theoretical Signal Production Cross Sections . . . . . . . . . . . . . . 127

7.5 Limit Calculation . . . . . . . . . . . . . . . . . . 127

8 Summary 131

8.1 Summary . . . . . . . . . . . . . . . . . . 131

A Figures for sub-samples 142

$\begin{array}{ll}\text { B Z' Signal Figures } & 148\end{array}$

C Tables for sub-samples $\quad 154$ 


\section{List of Tables}

2.1 The three generations of leptons . . . . . . . . . . . . 5

2.2 The three generations of quarks . . . . . . . . . . . . . 6

2.3 Gauge bosons in $\mathrm{SM} \ldots \ldots . \ldots . \ldots . \ldots 7$

3.1 Mass limits from previous studies for a $Z^{\prime} \ldots \ldots \ldots$

4.1 SMT sensor specifications . . . . . . . . . . . . . . . 30

4.2 Parameters of the Central Calorimeter . . . . . . . . . . . . . . . 42

4.3 Parameters of the End-Cap Calorimeter . . . . . . . . . . . . . . . . 42

6.1 List of DY MC samples and LO cross sections . . . . . . . . . . . 81

$6.2 Z$ peak fit results for fiducial sub-samples . . . . . . . . . . . . 90

6.3 Results of Gaussian fits to the various Z' MC data sets . . . . . . . . 103

6.4 Number of events above mass for combined CCCC topology . . . . . 110

6.5 Number of events above mass for combined CCCC and ECCC topology 111

7.1 Uncertainties of $Z^{\prime}$ signal efficiency due to PDFs for different mass points 121 
7.2 Breakdown of the integrated luminosity according to trigger version . 123

7.3 Number of events in asymmetric mass window for CCCC topology . . 124

7.4 Number of events in asymmetric mass window for ECCC topology . . 124

7.5 Number of events in asymmetric mass window for CCCC and ECCC topologies combined . . . . . . . . . . . . . . . . 125

7.6 Z' signal acceptance in CCCC topology . . . . . . . . . . . . . . 125

7.7 Z' signal acceptance in ECCC topology . . . . . . . . . . . . . . . 126

7.8 $\mathrm{Z}^{\prime}$ total signal acceptance for the total combined sample, CCCC and $\mathrm{ECCC} \ldots \ldots \ldots \ldots \ldots$

$7.9 \mathrm{Z}^{\prime}$ signal theoretical cross section . . . . . . . . . . . . . 127

C.1 Number of events above mass for both electrons in fiducial sub-sample 155

C.2 Number of events above mass for just one electron in fiducial sub-sample156

C.3 Number of events above mass for the ECCC topology . . . . . . . . . 157

C.4 Number of events in asymmetric mass window for both electrons in CCCC fiducial sub-sample . . . . . . . . . . . . . . . . . . . . 158

C.5 Number of events in asymmetric mass window for one electron in CCCC fiducial sub-sample . . . . . . . . . . . . . . . . . . . . . 158

C.6 Z' signal acceptance for both in fiducial in CCCC topology . . . . . . 159

C.7 Z' signal acceptance for only one electron in fiducial in CCCC topology 159 


\section{List of Figures}

2.1 The CTEQ6M PDF at $Q=100 \mathrm{GeV} \ldots \ldots . \ldots . \ldots . . \ldots 12$

4.1 Fermilab's accelerator chain . . . . . . . . . . . . . . . 21

4.2 Schematic of the D0 detector . . . . . . . . . . . . . 24

4.3 Schematic of the inner tracker . . . . . . . . . . . . . . . . 27

4.4 The silicon microstrip tracker . . . . . . . . . . . . . . . . . . . . 29

4.5 Cross-section of the scintillator strips used in the preshower detectors 33

4.6 FPS detector . . . . . . . . . . . . . . . . . . . . . 34

4.7 The calorimeters . . . . . . . . . . . . . . . . . . . . 39

4.8 A schematic of a calorimeter cell . . . . . . . . . . . . . . . . 40

4.9 Schematic view of the calorimeter showing pseudo-projective towers . 43

4.10 Calorimeter Electronics . . . . . . . . . . . . . . . . . 45

4.11 Muon system ....................... 46

4.12 LM location . . . . . . . . . . . . . . . . . . . . . . 48

4.13 Overview of the trigger and DAQ systems . . . . . . . . 50 
4.14 Overview of the L1 and L2 trigger systems . . . . . . . . . . . . . 51

5.1 The electron $f_{\mathrm{EM}}$ and $f_{\text {iso }}$ distributions . . . . . . . . . 67

5.2 Electron discrimination variables . . . . . . . . . . . . . . . . . . 72

5.3 Efficiencies for electrons with quality loose_notrk . . . . . . . . . . 74

5.4 Efficiencies for electrons with quality tight_notrk . . . . . . . . 75

5.5 Efficiencies for electrons with quality loose_trk . . . . . . . . 76

6.1 Comparisons of the $p_{T}$ spectrum of the $Z$ boson between data and MC, before and after correction . . . . . . . . . . . . . . 82

6.2 Dielectron invariant mass distribution of the DY background from MC 83

6.3 Invariant mass spectrum of events with misidentified electrons, as described in the text . . . . . . . . . . . . 86

6.4 Dielectron invariant mass distribution of data . . . . . . . . . . 87

6.5 Invariant mass distribution around $Z$ peak fitted with a Breit-Wigner convoluted with a Gaussian . . . . . . . . . . . . . . . . . . 89

6.6 Invariant mass distribution around $Z$ peak fitted with a Breit-Wigner convoluted with a Gaussian for the CCCC sub-samples . . . . . . . . 91

6.7 Invariant mass distribution around $Z$ peak fitted with a Breit-Wigner convoluted with a Gaussian for the ECCC sub-samples . . . . . . . . 92

6.8 Correction $\alpha=E_{\text {cor }} / E_{\text {uncor }}$ as a function of uncorrected energy $E_{u} n c o r$ for non-fiducial electrons, and fit results . . . . . . . . . . . . . . 94

6.9 Correction $\alpha^{\prime}=E_{c o r} / E_{\text {uncor }}$ as a function of detector $\left|\eta_{E C}\right| \ldots \ldots 6$ 
6.10 Data/Background comparison of invariant mass distribution for CCCC around $Z$ peak, combining "both in fiducial" and "one in fiducial" sub-

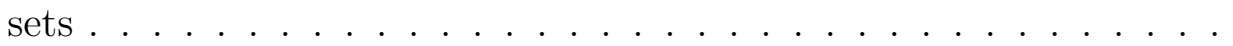

6.11 Data/Background comparison of invariant mass distribution around $Z$ peak, combining CCCC and ECCC topologies . . . . . . . . . . . . 101

6.12 Efficiency of the $\sqrt{\hat{s}}$ cut . . . . . . . . . . . . . . . . . 102

6.13 Results of the Gaussian fit applied to $\mathrm{Z}^{\prime}$ signal as a function of the generated $Z^{\prime}$ mass . . . . . . . . . . . . . . . . . . . . . . . . . . . . . 104

6.14 Reconstructed MC invariant mass distribution for a potential Z' $850 \mathrm{GeV}$ signal fitted with a Gaussian . . . . . . . . . . . . . . . . 105

6.15 Geometric acceptance and efficiency for each sub-sample used, for each $\mathrm{Z}^{\prime}$ mass point in an asymmetric window from $-3 \sigma$ to infinity . . . . 106

6.16 Total acceptance for each sub-sample, for each $\mathrm{Z}^{\prime}$ mass point in an asymmetric window from $-3 \sigma$ to infinity . . . . . . . . . . . . . 107

6.17 Data/Background comparison of the full range invariant mass distribution in CCCC, combining "both in fiducial" and "one in fiducial" sub-sets . . . . . . . . . . . . . . . . . . 109

6.18 Data/Background comparison of Invariant Mass distribution extrapolated to high masses, combining CCCC and ECCC topologies . . . . 112

7.1 NLO and NNLO $k$-factors for a sequential $\mathrm{Z}^{\prime}$ as a function of the invariant mass of the electron pair . . . . . . . . . . . . . . . . . 121

$7.295 \%$ CL limit on $\sigma \times B R\left(\mathrm{Z}^{\prime} \rightarrow e^{+} e^{-}\right)$for CCCC, and ECCC . . . . . 129 
7.3 95\% CL limit on $\sigma \times B R\left(\mathrm{Z}^{\prime} \rightarrow e^{+} e^{-}\right)$for the total combined sample, CCCC and ECCC . . . . . . . . . . . . . . . . . 130

A.1 Data/Background comparison of Invariant Mass distribution for both electron in fiducial in Central Calorimeter around $Z$ peak . . . . . . . 143

A.2 Data/Background comparison of Invariant Mass distribution for just one electron in fiducial in Central Calorimeter around $Z$ peak . . . . 144

A.3 Data/Background comparison of Invariant Mass distribution in ECCC around $Z$ peak, for the "in fiducial" sub-set . . . . . . . . . . . . . . 145

A.4 Data/Background comparison of the full range Invariant Mass distribution for "both in fiducial" and "one in fiducial" CCCC subsamples 146

A.5 Data/Background comparison of the full range Invariant Mass distribution for "in fiducial" ECCC subsample . . . . . . . . . . . . . . . . 147

B.1 Reconstructed MC invariant mass distribution for a potential Z' $400 \mathrm{GeV}$ signal fitted with a Gaussian . . . . . . . . . . . . . . . . . 149

B.2 Reconstructed MC invariant mass distribution for a potential Z' $500 \mathrm{GeV}$ signal fitted with a Gaussian . . . . . . . . . . . . . . . . . . 149

B.3 Reconstructed MC invariant mass distribution for a potential Z' $600 \mathrm{GeV}$ signal fitted with a Gaussian . . . . . . . . . . . . . . . . . 150

B.4 Reconstructed MC invariant mass distribution for a potential Z' $650 \mathrm{GeV}$ signal fitted with a Gaussian . . . . . . . . . . . . . . . . . . . 150

B.5 Reconstructed MC invariant mass distribution for a potential Z' $700 \mathrm{GeV}$ signal fitted with a Gaussian . . . . . . . . . . . . . . . . . . 151 
B.6 Reconstructed MC invariant mass distribution for a potential Z' $750 \mathrm{GeV}$ signal fitted with a Gaussian . . . . . . . . . . . . . . . . . 151

B.7 Reconstructed MC invariant mass distribution for a potential Z' $800 \mathrm{GeV}$ signal fitted with a Gaussian . . . . . . . . . . . . . . . . . 152

B.8 Reconstructed MC invariant mass distribution for a potential Z' $850 \mathrm{GeV}$ signal fitted with a Gaussian . . . . . . . . . . . . . . . . . . 152

B.9 Reconstructed MC invariant mass distribution for a potential Z' $900 \mathrm{GeV}$ signal fitted with a Gaussian . . . . . . . . . . . . . . . . . . 153

B.10 Reconstructed MC invariant mass distribution for a potential Z' $1000 \mathrm{GeV}$ signal fitted with a Gaussian . . . . . . . . . . . . . . . 153 


\section{ACKNOWLEDGEMENTS}

First and foremost I would like to express my gratitude to my advisor, Professor John Parsons for his invaluable support, supervision and useful suggestions throughout this research work. His continuous guidance has enabled me to complete my graduate studies successfully. The skills and knowledge that I have received from him

will continue to benefit me a lot in my future. I would also like to extend my thanks to the other Columbia professors on the D0 experiment, Mike Tuts and Gustaaf Brooijmans, for their help and guidance.

Secondly I would like to thank my committee members for a careful reading of my thesis: Professors Mike Shaevitz, Eduardo Ponton, Tony Heinz, and Dr. Francesco Lanni.

A special thank you for his help, guidance, and support during the early years of my graduate studies is owed to Professor Alan Blaer. His love for physics and his passion for teaching has always been a source of inspiration.

I would also like to thank Stefan Simion and the rest of the Nevis Laboratories electronics team with whom I worked on the ATLAS readout electronics and Front End Board. It was my first ever involvement in developing and debugging electronics, and they made it an enjoyable and effective experience. I would like to thank Abid Patwa for his guidance and cooperation in calibrating the D0 Forward Pre-shower. Additionally, the New Phenomena group, and especially the High $p_{T}$ Leptons subgroup deserve a special appreciation: Jean-Francois Grivaz and Arnd Meyer for always being available to offer their advice and opinion; Amitabha Das and Pieter van den Berg for providing help with analysis related software problems. I thank Emmanuel Tsesmelis for introducing me to high energy physics research methods, and being a 
mentor and good friend.

I would also like to thank Peter Russo, Jeremy Carlo, Saul Cohen, Idan Mandelbaum, Raphael Galea, and Dominik Dannheim for making my time in New York enjoyable. The same goes to Jovan Mitrevski, Tulika Bose, Kevin Black, Paul Russo, Lidija and Sasa Zivkovic, Sabine Lammers, Jan Petersen and Reinhard Schwienhorst for helping me to adjust to Fermilab, and making my stay there more enjoyable.

A thank you to my four-legged furry friends, Mugsy and Lily, for helping me keep perspective during the writing of this dissertation.

Heather, thank you for your love and support. You have been simply wonderful.

Finally, I would like to thank my parents. I would not be writing this dissertation if it weren't for their love, continuous support, dedication, and having instilled a creative spark to understand how the world works. 
To my parents... 


\section{Chapter 1}

\section{Introduction}

The Standard Model (SM) of particle physics is the best theory that physicists currently have to describe elementary particles and their interactions. It was developed in the early 70's and incorporates the strong, weak and electromagnetic forces. The SM includes Quantum Chromodynamics (QCD) for the strong force, and the weak and electromagnetic forces are unified under the Glashow-Weinberg-Salam model. Predictions of the SM are being matched by experimental data with astonishing precision [1]. For that reason, the SM is considered one of the biggest achievements in twentieth-century science.

However, the SM does not incorporate gravity, and even though this can be an excellent approximation, it cannot be the final theory of the fundamental particles and their interactions. Furthermore, it contains 19 free parameters that need to be empirically determined, rather than being derived from first principles. As a result various extensions of the SM have been developed. Many extensions rely on the gauge structure of the SM and expand it into larger groups, a fact that predicts the existence of new particles. This thesis searches for such a new particle, denoted as $\mathrm{Z}^{\prime}$, that is 
predicted by such extensions.

The outline of the thesis is as follows. Chapter 2 gives a short theoretical introduction to the Standard Model and discusses some of its weaknesses. Chapter 3 lists some of the $\mathrm{Z}^{\prime}$ models, and discusses the phenomenology of some popular models. Following in Chapter 4 is a description of the experimental apparatus; the Fermilab Tevatron Collider, and the D0 detector. Additionally, there is a short description of the data acquisition process. Chapter 5 discusses how the raw data collected with the detector is processed to identify physics objects and to measure their properties. Chapters 6 and 7 discuss the analysis; Chapter 6 presents the data selection and treatment, together with the background normalization, and the handling of the signal. Chapter 7 covers the limit setting procedure, and the parameters that are involved in the calculation of the limit. Chapter 8 summarizes the results of this analysis.

Throughout this dissertation, natural units are used. This system of units is defined by setting $\hbar=c=1$. In this system of units, a single unit is needed to express the mass, energy, and momentum. In high energy physics this unit is the $\mathrm{GeV}$ that is used throughout the dissertation. 


\section{Chapter 2}

\section{The Standard Model}

\subsection{The Standard Model}

The standard model of particle physics $(\mathrm{SM})^{1}$ is a quantum field theory that provides a description of the current understanding of the fundamental particles and their interactions, with the exception of gravity. Fundamental particles can be classified into fermions and bosons. Fermions have half-integer spin and make up all known matter in the universe (quarks and leptons). Each fermion has a corresponding anti-particle, having the same mass and spin but opposite sign of all internal quantum numbers. Interactions between these particles are mediated through the second set of elementary particles, bosons, which carry integer spin. The fundamental interactions are described by four forces: electromagnetic, weak, strong and gravitational. The SM describes the first three of these forces, treating each particle in terms of a mathematical field. It is based on the local symmetry group $S U(3)_{C} \otimes S U(2)_{L} \otimes U(1)_{Y}{ }^{2}$ that

\footnotetext{
${ }^{1}$ This discussion is based on the descriptions in $[2,3,4,5,6]$.

${ }^{2} \mathrm{C}$ corresponds to color, L to weak-isospin, and $\mathrm{Y}$ to weak-hypercharge
} 
describes strong, weak, and electromagnetic interactions, via the exchange of the corresponding spin-1 gauge fields: eight massless gluons and one massless photon, for the strong and the electromagnetic interactions, respectively, and three massive bosons, $W^{ \pm}$and $Z$ for the weak interaction.

\subsubsection{Fermions}

\section{Leptons}

The leptons, fermions that do not experience the strong force, are divided into three generations (or families), with corresponding particles across generations having similar properties, but different mass. The first generation consists of the electron $(e)$ and electron neutrino $\left(\nu_{e}\right)$, the second generation of the muon $(\mu)$ and muon neutrino $\left(\mu_{e}\right)$, and the third generation of the tau $(\tau)$ and tau neutrino $\left(\nu_{\tau}\right)$. Each family has an additive lepton quantum number associated with it; $L_{e}, L_{\mu}$, and $L_{\tau}$ respectively. Associated with the leptons are two additional quantum numbers: weak-isospin $(T)$, whose third component is denoted as $T^{3}$, and weak-hypercharge $(Y)$. In Table 2.1 the types of leptons, along with their electric charges and masses [7], are listed. The SM assumes that neutrinos are massless. However, recent experiments indicate that the neutrinos have mass $[8,9]$. As an immediate consequence, the lepton quantum numbers are not conserved in weak interactions. There also exist the charge conjugates to the leptons; the antileptons. These are the positron, the antimuon, the antitau, and the corresponding antineutrinos. Charge conjugation conserves variables such as spin and momentum, but changes the sign of all intrinsic additive quantum numbers, like electric charge and lepton number. Whether the neutrino is its own antiparticle is not yet known, and is the subject of investigation [10]. 


\begin{tabular}{|cccc|}
\hline Particle Name & Symbol & Charge $(\mathrm{e})$ & Mass $(\mathrm{MeV})$ \\
\hline \hline electron neutrino & $\nu_{e}$ & 0 & $<0.000002$ \\
electron & $e$ & -1 & 0.511 \\
\hline muon neutrino & $\mu_{\nu}$ & 0 & $<0.19$ \\
muon & $\mu$ & -1 & 105.7 \\
\hline tau neutrino & $\nu_{\tau}$ & 0 & $<18.2$ \\
tau & $\tau$ & -1 & 1777 \\
\hline
\end{tabular}

Table 2.1: The three generations of leptons.

\section{Quarks}

Quarks, which interact through the electromagnetic, weak, and strong forces, are divided into six "flavors", representing quantum numbers that are conserved under the strong and electromagnetic, but not weak, interactions. Like leptons, the quarks are divided into three generations (or families). The first generation consists of the up $(u)$ and down $(d)$ quark. The charm $(c)$ and strange $(s)$ quarks comprise the second generation, while the top $(t)$ and the bottom $(b)$ quark form the third generation. Quarks, have weak-hypercharge $(Y)$ and weak-isospin $(T)$ quantum numbers. Additionally, quarks have a quantum number associated with the strong force, which allows for the existence of baryons containing three quarks of the same flavor with parallel spins, forbidden by the Pauli exclusion principle. This quantum number is called "color charge" and is a gauge $S U(3)$ symmetry. Therefore quarks come in three colors; red $(R)$, green $(G)$, and blue $(B)$. Table 2.2 lists the types of quarks, along with their electric charges and masses [7]. 


\begin{tabular}{|cccc|}
\hline Particle Name & Symbol & Charge (e) & Mass $(\mathrm{MeV})$ \\
\hline \hline up & $u$ & $2 / 3$ & 1.5 to 3.0 \\
down & $d$ & $-1 / 3$ & 3 to 7 \\
\hline charm & $c$ & $2 / 3$ & 1250 \\
strange & $s$ & $-1 / 3$ & 95 \\
\hline top & $t$ & $2 / 3$ & 174200 \\
bottom & $b$ & $-1 / 3$ & 4200 \\
\hline
\end{tabular}

Table 2.2: The three generations of quarks.

\subsubsection{Gauge Bosons}

The interactions of elementary particles occur through exchange of gauge bosons, with the coupling displaying a gauge symmetry. These are the propagators of the fundamental forces in the SM. For the strong interaction, there are eight massless, colored gluons, coupling to the color $S U(3)_{C}$ charge. Conventionally they are named $g_{i}$, where $i=1 \ldots 8$ and corresponds to the $3^{2}-1$ generators of the $S U(3)$ symmetry group. For the weak interactions there are two charged $W$ bosons $\left(W^{ \pm}\right)$and a neutral $Z^{0}$ boson. They correspond to the three generators of $S U(2)$ of the weak interaction. For the electromagnetic interactions, the massless photon $(\gamma)$ is the carrier and correspond to the gauge group $U(1)$. Table 2.3 lists the force carriers in terms of their interaction type, along with their electric charges and masses [7].

The SM has one final boson: the Higgs boson. The interactions of the SM are introduced by demanding a gauge symmetry. Preserving this symmetry requires the corresponding boson to be massless as in the case of the photon and the gluons. However, the carriers of the weak interaction (the $W^{ \pm}$and the $Z^{0}$ ) have non-zero masses. The Higgs mechanism is the SM's way of avoiding this issue. By introducing a new scalar field, the interactions with the $W^{ \pm}$and the $Z^{0}$ can be constructed such 


\begin{tabular}{|cccc|}
\hline Force Carrier & Force & Charge $(\mathrm{e})$ & Mass $(\mathrm{GeV})$ \\
\hline \hline Gluon $(\mathrm{g})$ & Strong & 0 & 0 \\
\hline$W^{+}$ & & 1 & $80.403 \pm 0.029$ \\
$W^{-}$ & Weak & -1 & $80.403 \pm 0.029$ \\
$Z^{0}$ & & 0 & $91.188 \pm 0.002$ \\
\hline Photon $(\gamma)$ & EM & 0 & 0 \\
\hline
\end{tabular}

Table 2.3: Gauge bosons in SM.

that they acquire mass. The Higgs mechanism is also the method responsible for the fermions acquiring mass. This process explicitly breaks the symmetry of the interactions, and hence is called electroweak symmetry breaking [2, 6]. However, despite decades of direct and indirect searches the associated Higgs boson predicted by the SM has yet to be discovered.

\subsubsection{Quantum Electrodynamics (QED)}

Quantum Electrodynamics (QED) describes the interaction of fermions through the exchange of photons. The magnitude of these interactions can be calculated using perturbation theory, and can predict the probability of a particular, experimentally verifiable, outcome. Predictions of QED agree with experiment with an accuracy of $\sim 10^{-12}[11]$, making it the most accurate particle theory constructed to date. The Lagrangian for a free Dirac field $\Psi$ for a fermion, having mass $m$, is given by:

$$
\mathcal{L}=\bar{\Psi}\left(i \gamma^{\mu} \partial_{\mu}-m\right) \Psi
$$

where $\gamma^{\mu}$ are the Dirac matrices. This Lagrangian is invariant under global phase transformations $U(1)$, and the requirement of local gauge invariance results in the 
introduction of a vector field $A_{\mu}$, identified with the photon. The total Lagrangian, shown in Equation 2.2, is obtained through the addition of a kinetic term to account for the propagation of the vector field, which is also gauge invariant.

$$
\mathcal{L}=\bar{\Psi}\left(i \gamma^{\mu} \mathcal{D}_{\mu}-m\right) \Psi-\frac{1}{4} F_{\mu \nu} F^{\mu \nu}
$$

where $\mathcal{D}_{\mu} \equiv \partial_{\mu}+i q A_{\mu}$ is called the covariant derivative, and the term $F_{\mu \nu}=\partial_{\mu} A_{\nu}-$ $\partial_{\nu} A_{\mu}$ has been added to make $A^{\mu}$ be a dynamic variable of the Lagrangian. To preserve invariance under local gauge transformations, the gauge field (photon) is required to be massless, consistent with experimental observations.

\subsubsection{Electroweak}

The electrodynamic and weak interactions are unified in electroweak theory [12]. While for low energies they appear as two distinct forces, they are two aspects of the same force. Above the unification energy $\left(\sim 10^{3} \mathrm{GeV}\right)$, they combine into the electroweak force. The weak group symmetry $S U(2)_{L}$, where $L$ indicates that the weak bosons couple only to left-handed fermions, must be preserved when constructing the isospin triplet of weak currents. Therefore, it is necessary to modify the $U(1)$ electromagnetic group generator to account for right-handed interactions. The electric charge $U(1)$ group generator is replaced then with the hypercharge $\mathrm{Y}$, that is defined as

$$
Q=T_{3}+\frac{Y}{2}
$$

The theory requires weak isospin and hypercharge to be conserved, with the Lagrangian invariant under the local gauge transformation $S U(2)_{L} \otimes U(1)_{Y}$. The funda- 
mental vector bosons of the group are massless isovector triplets $W_{\mu}^{i}(i=1,2,3)$ for the $S U(2)_{L}$ group and massless isosinglet $B_{\mu}$ for the $U(1)_{Y}$ group. As has been mentioned before, the non-zero masses of the weak gauge bosons require the mechanism of spontaneous symmetry breaking, as the addition of a mass term to the Lagrangian, even its simplest form $(-m \psi \psi)$, would break the gauge invariance. An additional consequence of symmetry breaking is the existence of a new Higgs particle, as yet experimentally unconfirmed. The Higgs mechanism, described in more detail below, then gives rise to the large masses of the weak bosons.

\section{Electroweak Symmetry Breaking}

Spontaneous Symmetry Breaking (SSB) introduces a term to the Lagrangian that preserves the symmetry but allows the selection of a mass scale. A complex doublet of scalar fields is introduced, adding a potential to the Lagrangian:

$$
V=\lambda\left(\phi^{\dagger} \phi\right)^{2}-\mu^{2} \phi^{\dagger} \phi
$$

where $\phi$ indicates the scalar field which has a non-zero minimum at $|\phi|=\sqrt{\mu^{2} / 2 \lambda} \equiv$

$v / \sqrt{2}$ for $\lambda, \mu>0$. It is this acquisition of a non-zero vacuum expectation value (VEV) $(246 \mathrm{GeV})$ that spontaneously breaks the electroweak gauge symmetry. The quadratic terms in the physical boson field, shown in Equation 2.5, then give the $W$ and $Z$ bosons their masses, shown in Equation 2.6.

$$
\begin{gathered}
\mathcal{L}_{\text {mass }}=\frac{g^{2} v^{2}}{4} W_{m}^{+} W_{m}^{-}+\frac{\left(g^{2}+g^{\prime 2}\right) v^{2}}{8} Z_{\mu} Z^{\mu} \\
m_{W}=\frac{1}{2} v g \text { and } m_{Z}=\frac{1}{2} v \sqrt{g^{2}+g^{\prime 2}} \equiv \frac{m_{W}}{\cos \theta_{W}}
\end{gathered}
$$


The existence of the non-zero VEV also gives rise to the fermion masses. After having introduced the additional scalar doublet into the model, the Yukawa-type Lagrangian after SSB can take the form:

$$
\mathcal{L}_{Y}=-\frac{1}{\sqrt{2}}(v+H)\left\{c_{1} \bar{d} d+c_{2} \bar{u} u+c_{3} \bar{e} e\right\}
$$

Thus, the SSB mechanism generates the fermion masses:

$$
m_{d}=\frac{1}{\sqrt{2}} c_{1} v, m_{u}=\frac{1}{\sqrt{2}} c_{2} v \text { and } m_{e}=\frac{1}{\sqrt{2}} c_{3}
$$

where $c_{1}, c_{2}, c_{3}$ are the Yukawa couplings. Since the values of parameters $c_{i}$ are not known, the fermion masses are arbitrary.

The SSB Lagrangian introduces a new scalar particle into the model, the Higgs boson that its mass is calculated to be:

$$
M_{H}=\sqrt{2 \lambda} v .
$$

Thus far the experimental searches of the Higgs have provided a lower limit on its mass $M_{H}>114.4 \mathrm{GeV}[7]$.

\subsubsection{Quantum Chromodynamics (QCD)}

Quantum Chromodynamics (QCD) is the quantum field theory describing the interactions of the quarks and gluons (partons), and differs from QED in its non-Abelian nature (the gluon exhibits self interaction). To explain the experimental observations of hadron spectroscopy, a three-fold color degree of freedom is introduced, each quark 
carrying a single color, so giving three quarks of each flavor, for a total of 18 quarks. An octet of bicolored gluons then mediate the interactions between the quarks. The color singlet does not contribute to strong interactions as it is colorless, so is unable to mediate forces between color charges. Emission of a gluon may lead to a quark changing color, but the color of the entire system is conserved. The Lagrangian must be constructed to be invariant under both global and local gauge transformations. The strong interaction is described using the $S U(3)_{\text {color }}$ gauge symmetry, which introduces eight massless vector fields, the gluons.

The composite structure of hadrons must be accounted for when predicting interactions between them. The valence quarks, which define the quantum numbers, sea quarks (virtual quark-antiquark pairs produced from the splitting of gluons), and the gluons themselves are referred to as partons, and are described by parton distribution functions (PDFs). The PDF gives the probability that a specific parton will have a fraction $x$ of the hadron momentum. The PDF is needed whenever cross sections involving high-energy interactions with hadrons are calculated. In particular, the PDF distribution, $f(x, Q) d x$, is the number of quarks or gluons of a particular type $(u, d$, etc.) in a particular type of hadron (proton, pion, etc) carrying a momentum fraction between $x$ and $(x+d x)$ of the hadron's momentum in the infinite momentum frame, when probed by an interaction with $Q$ momentum transfer. Figure 2.1 gives the PDF for protons for $Q=100 \mathrm{GeV}$ from the CTEQ6M NLO parametrization [13].

\subsubsection{Limitations of the Standard Model}

The SM predictions show excellent agreement with experimental data from high energy experiments. However, it is not a complete theory since it does not include the dominant force in the macroscopic world, gravity. Furthermore, it fails to answer 


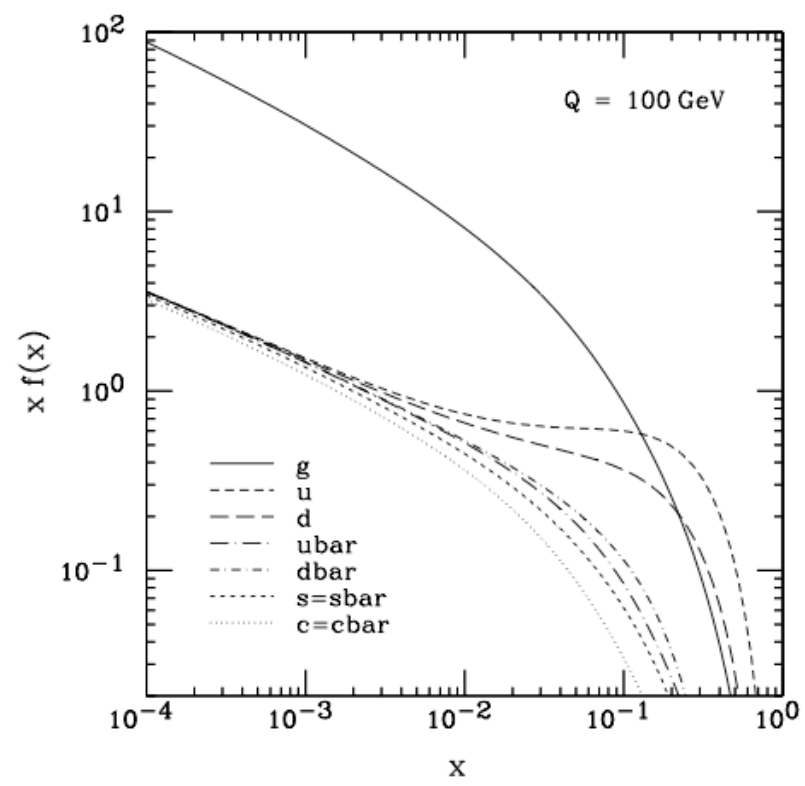

Figure 2.1: The CTEQ6M parton distribution function at $Q=100 \mathrm{GeV}$. The value $f(x) d x$ is the number of quarks or gluons of the particular type in a proton carrying a momentum fraction between $x$ and $(x+d x)$ of the proton's momentum. (Figure from Ref. [13].)

questions like why the fermions have the observed masses, the origin of flavor, and what makes up dark matter. The strong and electroweak interactions are not unified under its framework. The SM contains 19 free, empirically determined, parameters.

The SM fails to address the so-called hierarchy problem. The hierarchy problem arises from the fact that the weak scale $\left(M_{\text {weak }} \sim 100 \mathrm{GeV}\right)$ and the Planck scale $\left(M_{P} \sim 10^{19} \mathrm{GeV}\right)$ differ by 17 orders of magnitude. The Higgs mass is quadratically divergent when one loop self-interactions of the Higgs boson are considered. For these divergences to be canceled an additional mass counterterm, $\delta m_{h}^{2}$, needs to be introduced. In the lowest order in perturbation theory, the Higgs mass is $m_{H}^{2}=m_{0}^{2}+$ 
$\delta m_{H}^{2} \approx m_{0}^{2}-g^{2} \Lambda^{2}$, where $m_{0}$ is the "ground" Higgs mass, $g$ is a dimensionless coupling constant, and $\Lambda$ is the energy scale. Taking into account that recent bounds on the Higgs mass [14] are on the order of the electroweak scale, and assuming that $g \approx 1$ and $\Lambda$ is around the Planck scale, then $m_{0}$ must be adjusted so that $m_{0}^{2}-g^{2} \Lambda^{2} \approx m_{H}^{2}$. This requires a precise adjustment of the SM parameters and is referred to as the fine tuning problem.

In order to address the above issues, many theories have been developed beyond the Standard Model. A popular theory is Supersymmetry (SUSY). More relevant to this analysis are theories that consider larger unification groups like Grand Unified theories (GUT's) with gauge groups larger than $S U(5)$ that give rise to at least one extra gauge neutral boson $\left(Z^{\prime}\right)$. 


\section{Chapter 3}

\section{$Z^{\prime}$ Models and Phenomenology}

An open question in particle physics is if there are any additional gauge bosons beyond the ones associated with the SM gauge group structure. This question is intriguing given that the selection of the gauge bosons observed so far remains a mystery. Additionally, new gauge bosons are predicted in many theories beyond the SM that try to provide answers to its many open questions.

The simplest way of extending the SM gauge structure is to include an additional $U(1)$ group. Then the underlying effective gauge group at low energies $\left(E<<E_{G U T}\right)$ is:

$$
S U(3)_{C} \otimes S U(2)_{L} \otimes U(1)_{Y} \otimes U^{\prime}(1)
$$

That addition gives rise to an associated gauge boson, usually labeled as $\mathrm{Z}^{\prime}$, that is an electrically neutral, spin-1 particle. In most extended gauge theories the symmetry breaking scale is at sufficiently high energies that the associated extra bosons are beyond the reach of current or planned experiments. However, there exist several models that allow a relatively light $\mathrm{Z}^{\prime}$. If the new gauge coupling is not much smaller 
than unity, then the $U(1)$ group must be spontaneously broken at a scale larger than the electroweak scale to account for the non-observation of the $\mathrm{Z}^{\prime}$ boson at LEP and during Run I of the Tevatron [15]. Such models can have a $Z^{\prime}$ that is on the order of $\mathrm{TeV}$ scale and is in the reach of current experiments. In the following sections there will be an overview of some of these models, like Grand Unification Theories, Left - Right Symmetric Model, Superstring theories and the Sequential Standard Model, and a review of the results from previous $Z^{\prime}$ searches.

There are various other models that predict extra neutral gauge bosons, such as the Alternative Left-Right Model (ALRM), un-unified Standard Model (UNSM), the Little Higgs scenario, the BESS model. Descriptions of these models can be found at $[16,17,18,19]$

\section{1 $\quad Z^{\prime}$ Models}

\subsubsection{Grand Unification Theories}

In general, GUTs propose that strong and electroweak interactions can be combined to a single interaction, and they have a single coupling at a unification scale $E_{G U T}$. GUTs make predictions that can be tested in experiments. Such a prediction is that the proton must decay. This decay is mediated by the exchange of gauge bosons with a mass at the scale of $E_{G U T}$. To be consistent with the present limits on proton decay, the unification scale must be $E_{G U T}>10^{15} \mathrm{GeV}$. GUTs postulate a new spontaneous symmetry breaking similar to the electroweak one, in order to account for the different coupling observed in low $\left(E<<E_{G U T}\right)$ energies. The simplest group constructed that contains the SM gauge symmetry is based on $S U(5)$. This model has been ruled out because it requires a proton decay time that has been experimentally excluded. 
Two popular examples of GUTs originate from the groups $S O(10)$ and $E_{6}$. The $S O(10)$ contains an extra $U(1)$ sub-group. $S O(10)$ is decomposed in sub-groups as: $S O(10) \rightarrow S U(5) \otimes U(1)_{\chi}$. Similarly, GUTs that originate from the $E_{6}$ group decompose in terms of the chain:

$$
E_{6} \rightarrow S O(10) \otimes U(1)_{\psi} \rightarrow S U(5) \otimes U(1)_{\chi} \otimes U(1)_{\psi} \rightarrow S M \otimes U(1)_{\theta_{E_{6}}}
$$

where $U(1)_{\theta_{E_{6}}}$ remains unbroken at low energies [16]. The corresponding neutral gauge bosons are denoted as $Z_{\chi}$ and $Z_{\psi}$.

\subsubsection{Left - Right Symmetric Model (LRM)}

One $S O(10)$ GUT extension to the SM postulates the existence of a right-handed version of the weak interaction:

$$
\begin{aligned}
S O(10) & \rightarrow S U(3)_{C} \otimes S U(2)_{L} \otimes U(1)_{Y} \otimes U(1)_{\chi} \\
& \rightarrow S U(3)_{C} \otimes S U(2)_{L} \otimes S U(2)_{R} \otimes U(1)_{B-L}
\end{aligned}
$$

The first chain leads to the additional neutral gauge boson $Z_{\chi}$ mentioned above, whereas the second chain gives the LRM that extends the SM gauge group to $S U(2)_{L} \otimes$ $S U(2)_{R} \otimes U(1)_{B-L}$ with an additional neutral gauge boson, denoted as $Z_{L R M}$ (as well as a right-handed charged boson). An interesting aspect of the LRM is that once the $S U(2)_{R}$ is introduced it contains right-handed neutrinos. That causes the generator of $U(1)$ to become baryon minus lepton number $(B-L)$, that is a physical observable. The LRM accommodates small masses for left handed neutrinos as well [20]. 


\subsubsection{Superstring Theory}

Supersymmetry (SUSY) and string theory are two popular extensions to the SM. SUSY introduces the concept of a relation between the bosons and the fermions in the SM. Each SM particle has a "superpartner" called "sparticle" with spin differing by $\frac{1}{2}$, whereas all the other quantum numbers remain the same. The inclusion of the "sparticles" into mass loop corrections results in a cancellation of the quadratic divergences inherent in the SM.

String theory is a popular theory that is able to include gravity. String theory describes all the fundamental matter particles as one dimensional strings, instead of zero dimensional points used in field theories. Various configurations of these strings can generate all types of fundamental particles found in nature, including a mediator for the gravitational force. String theory requires additional space-time dimensions beyond the four SM ones.

The supersymmetric version of string theory is called Superstring Theory [21]. The gauge group $E_{6}$ emerges in superstring theory when some of the higher dimensions are compactified. The $E_{6}$ contains $U(1)+\chi$ and $U(1)_{\psi}$ sub-groups, as has been mentioned earlier. The linear combination $\sqrt{3 / 8} Z_{\chi}-\sqrt{5 / 8} Z_{\psi}$ corresponds to the extra neutral gauge boson arising in some of the superstring models. Is it denoted as $Z_{\eta}$

\subsection{4 "Sequential" Standard Model}

The "sequential" Standard Model includes a neutral gauge boson $Z_{S M}^{\prime}$ with the same couplings to quarks and leptons as the SM $Z^{0}$ boson, and decays only to the three known families of fermions. Such a model is not gauge invariant [16], unless it has 
different couplings to exotic fermions, or if it occurs as an excited state of the ordinary $Z^{0}$ in models with extra dimensions at the weak scale [17]. However, it is a useful reference case when comparing constraints from various sources, and is traditionally used in the experimental analysis. For the remaining of this analysis, the symbols Z' and $Z_{S M}^{\prime}$ are considered equivalent.

The $\mathrm{Z}^{\prime}$ decay width is equal to the width of the SM $Z^{0}$ boson scaled by a factor of $M_{\mathrm{Z}^{\prime}} / M_{Z}$ :

$$
\Gamma_{Z^{\prime}}=\Gamma_{Z} \times \frac{M_{Z^{\prime}}}{M_{Z}}
$$

\subsection{Previous Z' Searches}

$\mathrm{Z}^{\prime}$ searches are of two kinds: indirect and direct. The indirect searches look for deviations from the SM that might be associated with the existence of a $Z^{\prime}$. Due to the presence of extra gauge groups, a mixing between the SM Z and the $\mathrm{Z}^{\prime}$ can happen. Changes in some of the measured values of SM parameters and observables result from this $Z-Z^{\prime}$ mixing. This usually involves precision electroweak measurements at, below, and above the $Z$-pole. Additionaly, precise measurements of the $W$ mass and weak neutral - current parameters can set limits in the amount of the $Z-Z^{\prime}$ mixing allowed. From these constraints on the $Z-Z^{\prime}$ mixing, limits on the $Z^{\prime}$ mass can be set [18].

In contrast, the direct searches rely on the explicit production of the $\mathrm{Z}^{\prime}$. Direct searches are categorized in terms of the initial state where a $Z^{\prime}$ is produced and the final state into which it decays. In $e^{+} e^{-}$colliders the process is $e^{+} e^{-} \rightarrow \mathrm{Z}^{\prime} \rightarrow$ $l^{+} l^{-}$, and for hadron colliders the process is: $p \bar{p} \rightarrow \mathrm{Z}^{\prime} \rightarrow l^{+} l^{-}$. The common final states examined are those involving two oppositely charged leptons, like dielectrons 
or dimuons. Other decay channels as di-jet, $t \bar{t}, e \mu$, or $\tau^{+} \tau^{-}$are also possible, but less distinct experimentally.

Direct searches from $e^{+} e^{-}$colliders have ruled out the possibility of a light $\mathrm{Z}^{\prime}$ with masses less than $200 \mathrm{GeV}$. For a heavy $\mathrm{Z}^{\prime}\left(M_{\mathrm{Z}^{\prime}}>>M_{Z}\right)$ the best direct limits come from $p \bar{p}$ colliders, where the $\mathrm{Z}^{\prime}$ is produced through the Drell-Yan process and decays to two leptons. In Table 3.1 the present $Z^{\prime}$ mass limits are summarized for the different models discussed above.

\begin{tabular}{|c|c|c|c|}
\hline \multirow{2}{*}{$Z^{\prime}$ Model } & \multicolumn{2}{|c|}{ Indirect Searches $(\mathrm{GeV})$} & Direct Searches $(\mathrm{GeV})$ \\
\cline { 2 - 3 } & Electroweak & LEP & \\
\hline$Z_{\chi}$ & 680 & 781 & 740 \\
$Z_{\psi}$ & 137 & 475 & 725 \\
$Z_{\eta}$ & 619 & 515 & 745 \\
$Z_{L R M}$ & 860 & 518 & 630 \\
$Z_{S M}^{\prime}$ & 1500 & 1305 & 850 \\
\hline
\end{tabular}

Table 3.1: Mass limits from previous studies for a $Z^{\prime}$. Results are taken from [7].

The most recent D0 published result is based on an integrated luminosity of $122 \mathrm{pb}^{-1}$, and sets a lower limit for a sequential $\mathrm{Z}^{\prime}$ at $719 \mathrm{GeV}$ (95\%C.L.) [22]. The other Tevatron detector, CDF, set a limit at $850 \mathrm{GeV}$ (95\%C.L.) using a dataset with integrated luminosity of about $450 \mathrm{pb}^{-1}[23]$. 


\section{Chapter 4}

\section{Experimental Apparatus}

\subsection{Accelerator Chain}

The Fermilab Tevatron Collider [24, 25, 26], which is located at the Fermi National Accelerator Laboratory, is the highest energy collider currently in operation, with a center of mass energy, $\sqrt{\mathrm{s}}$, of $1.96 \mathrm{TeV}$. The Tevatron is a proton-antiproton $(p \bar{p})$ collider, with beams of protons and antiprotons traveling in opposite directions around a circular ring of radius $1 \mathrm{~km}$.

The Tevatron has had two major periods of physics running, called Run I and Run II. Run I lasted from 1992 to 1996 , operating at $\sqrt{\mathrm{s}}=1.8 \mathrm{TeV}$. The accelerator and the detectors were then upgraded for higher energy and higher luminosity running. In March 2001, Run II commenced with the current $\sqrt{\mathrm{s}}=1.96 \mathrm{TeV}$.

Producing, injecting, and accelerating these beams is a complex process that takes place in many steps, and uses many different parts of the accelerator complex. Figure 4.1 shows the chain of accelerators involved in the process. 


\section{FERMILAB'S ACCELERATOR CHAIN}

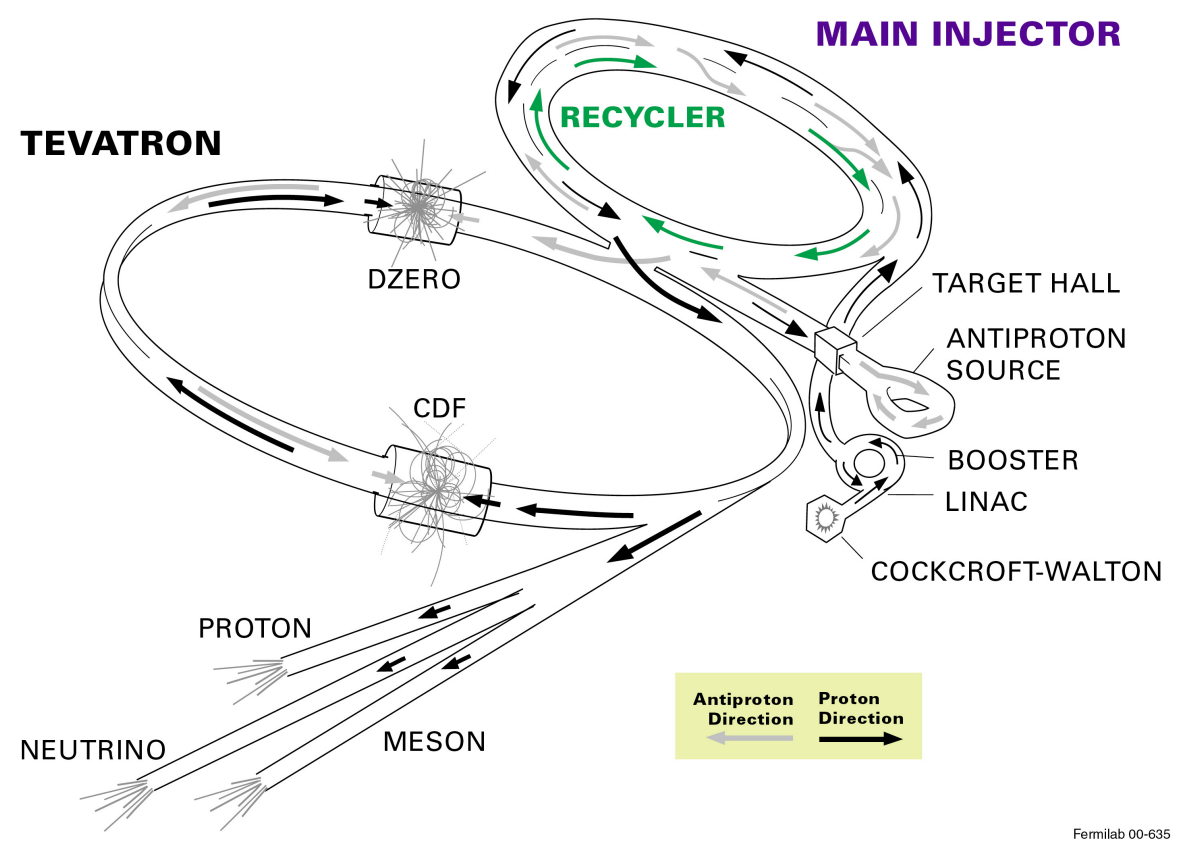

Figure 4.1: Fermilab's accelerator chain [29].

The beam starts as pressurized hydrogen gas. The hydrogen atoms are ionized by the addition of electrons, forming negative hydrogen $\left(\mathrm{H}^{-}\right)$ions. This process takes place at the magnetron surface-plasma source [24, 27]. The Magnetron consists of an oval cathode surrounded by an anode inside a magnetic field. Pressurized hydrogen gas $\left(H_{2}\right)$ is injected at one end. The cathode serves as the active surface for producing $H^{-}$ions which then form a dense plasma inside the magnetron. On the opposing end, an extractor plate accelerates the negative ions out of the source while the magnetic field steers electrons and other ions out of the $\mathrm{H}^{-}$source. The exiting $\mathrm{H}^{-}$ions have an energy of $18 \mathrm{KeV}$.

The magnetron source is surrounded by a Cockcroft-Walton generator [24, 28] that accelerates the $H^{-}$ions to an energy of $750 \mathrm{keV}$, at which point they enter the 
Linac $[28,30,31]$ for further acceleration.

The Linac is a two stage, $150 \mathrm{~m}$ long, linear accelerator, that accelerates the $H^{-}$ ions up to $400 \mathrm{MeV}$. The first stage, part of the original $200 \mathrm{MeV}$ Linac built in 1971, accelerates the ions to $116 \mathrm{MeV}$. It is approximately $80 \mathrm{~m}$ long and consists of a series of five radio frequency $(\mathrm{RF})$ tanks. Each tank consists of a series of alternating RF accelerating cavities and drift tubes. The second stage accelerates the ions up to $400 \mathrm{MeV}$. The reason for the Linac energy upgrade was to decrease beam losses in the Booster [24,32], the next step in the acceleration chain.

In the Booster, a $8 \mathrm{GeV}$ fast cycling proton synchrotron with a $151 \mathrm{~m}$ diameter, $H^{-}$ions are injected via a multi-turn charge-exchange injection [24, 33], which is the reason why $\mathrm{H}^{-}$ions are used in the beginning of the accelerator chain. After debunching the $H^{-}$ions from the Linac to minimize their momentum spread, the $H^{-}$ beam passes through a carbon foil which strips off the electrons, and is merged over multiple turns with the proton beam already in the Booster.

Protons are then delivered to the Main Injector [34]; a large aperture, rapid cycling, proton synchrotron with a circumference of $3320 \mathrm{~m}$. When the Tevatron operates in collider mode, the Main Injector serves two purposes. First it is used to raise the energy of the beam to $150 \mathrm{GeV}$ for injection into the Tevatron. Secondly, the Main Injector is used to accelerate protons up to $120 \mathrm{GeV}$, where they are extracted and directed on a nickel target for the purpose of creating antiprotons. The target is followed by a lithium lens to focus the secondary particles, and then a dipole magnet to select $8 \mathrm{GeV}$ antiprotons. These are then sent to the Debuncher, a triangular $8 \mathrm{GeV}$ synchrotron, to reduce the momentum spread. From there, the antiprotons are "stacked" in the Accumulator, another $8 \mathrm{GeV}$ synchrotron [24, 25, 35].

The limiting factor to the luminosity of the Tevatron has been the number of 
antiprotons available. Therefore, a recent addition has been the Recycler [36], an $8 \mathrm{GeV}$ storage ring with permanent magnets that shares the same tunnel as the Main Injector. When the Accumulator reaches its maximum capacity, the stacking efficiency decreases, so the antiprotons are transferred to the Recycler, and the stacking efficiency is improved. Additionally, electron cooling was recently accomplished to further improve the antiproton beam and potentially significantly improve the luminosity [37].

The final synchrotron, the Tevatron, receives protons from the Main Injector and antiprotons from the Accumulator or the Recycler and accelerates them up to $0.98 \mathrm{TeV}^{1}$. The Tevatron has a radius of $1 \mathrm{~km}$, and includes 774 dipoles, and 216 quadrapole magnets. Protons are accelerated in one direction while antiprotons are accelerated in the opposite direction around the ring. The Tevatron currently operates in a ' 36 on 36' mode where 36 bunches of protons and 36 bunches of antiprotons counter-circulate. In each proton bunch there are approximately $10^{11}$ protons and in each anti-proton bunch approximately $10^{10}$ antiprotons. There are currently two interaction regions where the bunches are made to collide every 396 ns. One of these regions has the label D0 and that is where one of two large collider detectors at Fermilab is located. At the other interaction region sits the second detector facility at Fermilab; CDF.

\subsection{The D0 Detector}

The D0 detector [38, 39], is a general purpose detector constructed to study $p \bar{p}$ collisions, especially high-mass states and phenomena with large transverse momentum.

\footnotetext{
${ }^{1}$ The original design specified an energy of $1 \mathrm{TeV}$. However, magnet stability issues dictated that the operation point of the Tevatron had to be lowered slightly.
} 


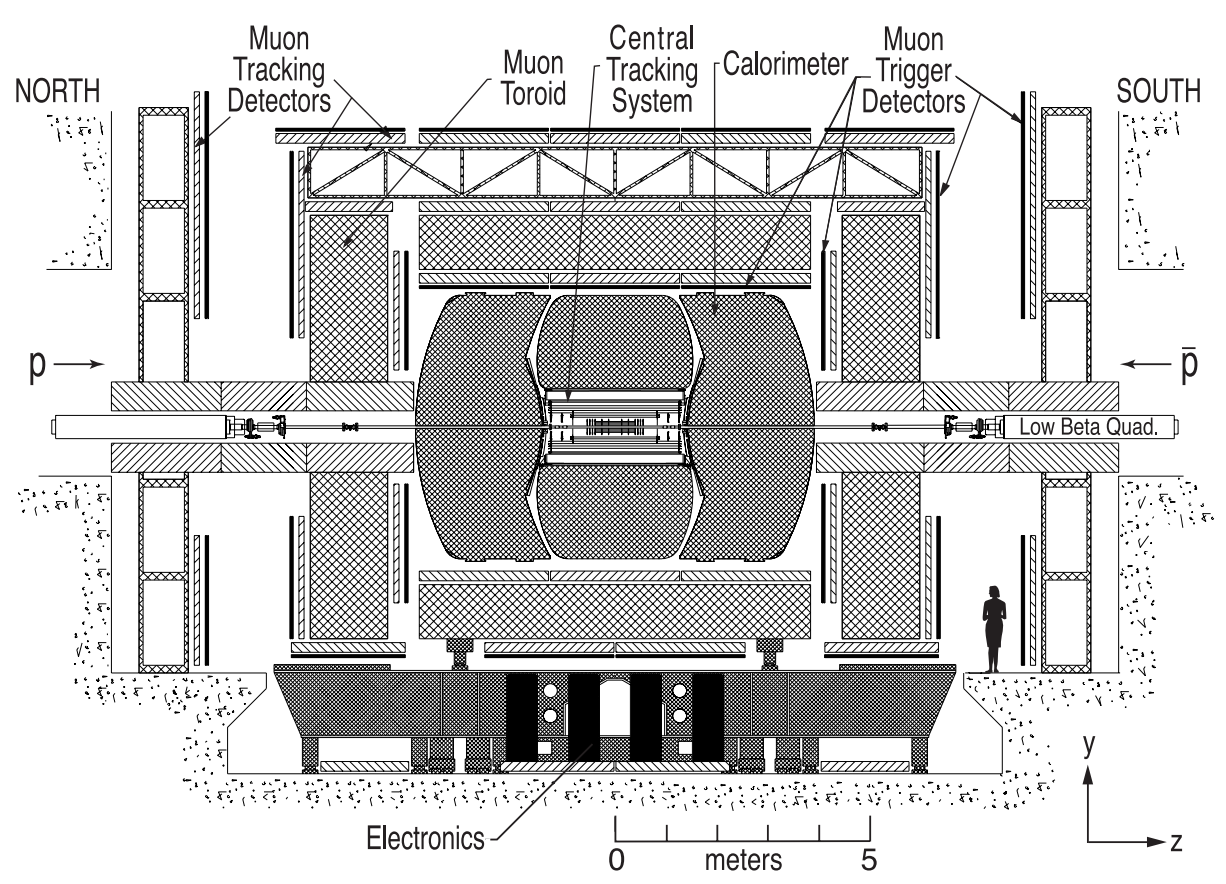

Figure 4.2: Schematic diagram of the RunII D0 detector [39].

The detector was initially commissioned in 1992 for Run I of the Tevatron, and it was significantly upgraded for the start of Run II in 2001. A description of the Run II version of the D0 detector follows.

Figure 4.2 shows a schematic diagram of the RunII D0 detector. Protons enter from the north side, and antiprotons from the south, colliding in the center inside a beryllium beam pipe which has a wall thickness of $0.508 \mathrm{~mm}$ and an outer diameter of $38.1 \mathrm{~mm}$. Closest to the collision point is the central tracking system, consisting of the silicon microstrip tracker (SMT) and the central fiber tracker (CFT), all inside a superconducting solenoidal magnet. Outside of the magnet are the preshower detectors, to compensate for the material in the tracking system, followed by the calorimeters. Finally, outside of the calorimeters is the muon system, with its toroidal magnets. 


\subsubsection{Coordinate System}

As indicated in Figure 4.2, a right-handed coordinate system is used for the detector and data analysis. The positive $z$ direction is aligned along the direction that beam protons travel, and the $y$ direction is set to be upwards. The $r$ coordinate is defined as in the cylindrical coordinate system: $r=\sqrt{x^{2}+y^{2}}$. The polar and azimuthal angles are written as $\theta$ and $\phi$, with the standard definitions. The origin can be chosen to be either in the middle of the detector or at the interaction vertex of a given event. When the origin is set in the middle of the detector, then the values are called detector values, while they are called physics values when the origin is set at the interaction vertex. Unless otherwise specified, when discussing physics the physics values are used, and when discussing the detectors the detector values are referenced. For $\theta$ and derived values the distinction can be large since, due to the length of the bunches, the interaction vertices are distributed in the $z$ coordinate with $\sigma_{z} \approx 25 \mathrm{~cm}$. Since $\phi_{\text {det }} \approx \phi_{\text {phys }}$, the distinction is often not made for $\phi$.

The pseudorapidity, $\eta$, is defined as:

$$
\eta=-\ln \left[\tan \left(\frac{\theta}{2}\right)\right],
$$

which approximates the true rapidity, $y$ (not to be confused with the coordinate $y$ ):

$$
y=\frac{1}{2} \ln \left(\frac{E+p_{z}}{E-p_{z}}\right)
$$

for relativistic particles. Differences of rapidity are invariant to boosts parallel to the beam line. The distinction is often made between $\eta_{\text {det }}$ and $\eta_{\text {phys }}$, as discussed above. The variable $\Delta R$, unless otherwise specified, is defined as $\sqrt{(\Delta \eta)^{2}+(\Delta \phi)^{2}}$. "Transverse" values, such as the transverse momentum $p_{\mathrm{T}}$, unless otherwise stated, 
are transverse relative to the beam line. Transverse values are often used since, due to the compositeness of the proton, the longitudinal energy of the collision is not known. The most commonly used transverse values are:

- $E_{T}=E \sin \theta:$ Transverse Energy,

- $p_{\mathrm{T}}=p \sin \theta=\sqrt{p_{x}^{2}+p_{y}^{2}}$ : Transverse momentum,

- $\mathbb{E}_{T}$ Missing transverse energy, or energy imbalance in the transverse plane.

\subsubsection{Central Tracking}

The central tracking system was newly designed for Run II and is composed of four elements: a Silicon Microstrip Tracker (SMT) [40], a Central Fiber Tracker (CFT) [41], a $2 \mathrm{~T}$ superconducting solenoidal magnet [42], and pre-shower detectors [43]. Figure 4.3 shows the location of the components.

The purpose of the central tracking system is to find and measure tracks of charged particles. Charged particles interact with the tracking detectors and leave a pattern of 'hits' in the various layers of the detectors. From these hits, a track can be reconstructed representing the trajectory of a charged particle. Since the entire tracking region is inside a highly uniform magnetic field, the trajectories of charged particles are curved. By measuring the curvature of the track, one can measure its momentum. Tracks in the central tracker can be used to aid in identification of charged particles by matching the tracks with information from the other subdetectors. 


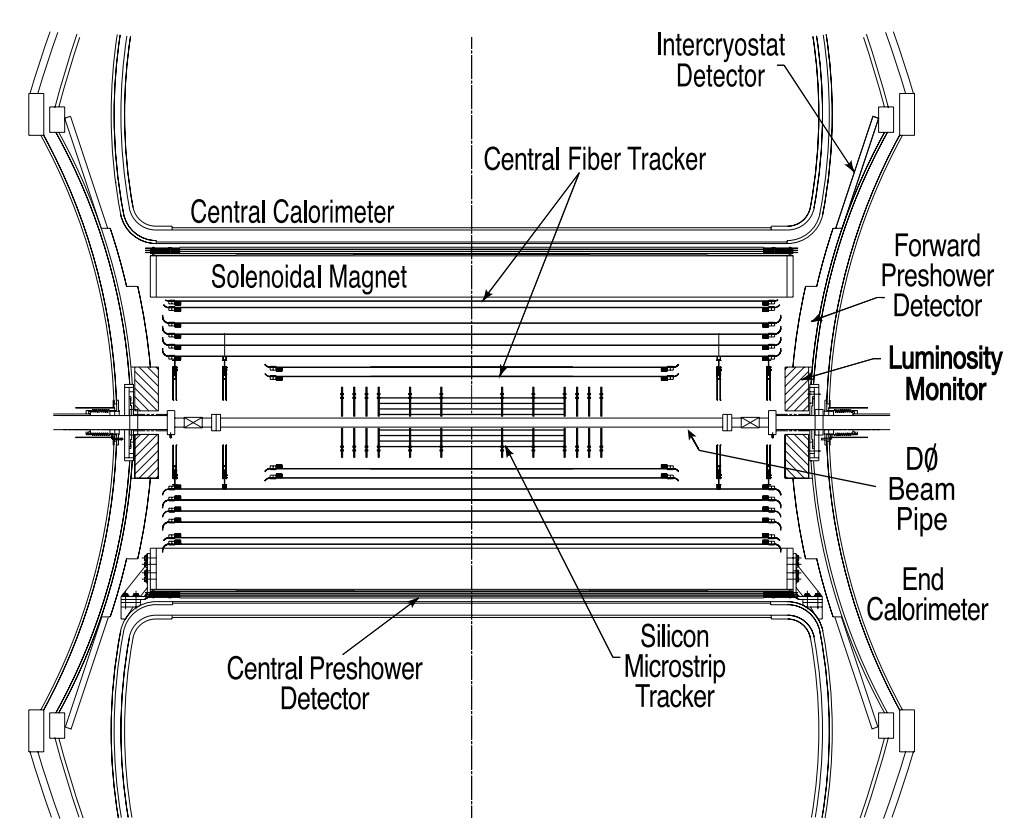

Figure 4.3: Schematic diagram of the inner tracker [39], and its surroundings

\section{Silicon Microstrip Tracker}

The SMT is the subdetector closest to the beam pipe, and provides both tracking and vertexing over nearly the full $\eta$ coverage of the calorimeter and muon systems. The design of the detector is primarily dictated by the accelerator environment. The length of the interaction region $\left(\sigma_{z} \approx 25 \mathrm{~cm}\right)$ sets the length scale of the SMT. The long interaction point complicates the design of the detector since it is desired to make the tracks cross perpendicular to the SMT surface for all $\eta$ [39].

The SMT uses silicon microstrip sensors to provide precise tracking close to the interaction point, necessary for precisely locating the primary interaction vertex as well as for reconstructing the secondary decay vertices of short-lived bottom hadrons. Silicon sensors work under the principle that charged particles going through silicon ionize the material, creating electron-hole pairs. By appropriately biasing the sensor, 
by reverse-biasing a $p n$-junction, a large depletion zone is created; an area that is depleted of free charge carriers such as electrons and holes. Since the depletion zone has an electric field across it, any electron-hole pairs created in the depletion zone are quickly swept out in opposite directions. Thus, if an ionizing particle goes through the depletion zone, the electron-hole pairs are quickly collected, providing a signal that can be read out from the electrodes. The probes collecting the charges can be made to be narrow strips, microstrips, in order to be able to measure spatially where the ionizing particle passed.

The SMT is constructed in three modules: six barrels which instrument the central detector, twelve $\mathrm{F}$ disks interspersed along the barrels, and four $\mathrm{H}$ disks which cover the far forward region. Figure 4.4 shows a three-dimensional representation of the SMT, and in Figure 4.3 the spatial relations of the SMT modules to each other and to the CFT, is shown. The SMT uses various types of sensors, some single sided (SS), with microstrips on one side only, and some double sided (DS), with microstrips on both sides. On DS detectors, the strips on each side can be at an angle relative to each other in order to be able to localize the path of the ionizing particle in more than one direction. However, that does lead to more complicated fabrication and lower yield, and possibly higher sensitivity to radiation damage. Specifications of the sensors used are given in Table 4.1, including the pitch between the strips and the angle between the strips. The six barrel detectors are centered at $|z|=6.2,19.0$, and $31.8 \mathrm{~cm}$; the twelve F-disks at $|z|=12.5,25.3,38.2,43.1,48.1$, and $53.1 \mathrm{~cm}$; and the four H-disks at $|z|=100.4$ and $121.0 \mathrm{~cm}$. The barrel detectors extend in the radial direction from $2.7 \mathrm{~cm}$ to $7.6 \mathrm{~cm}$, the $\mathrm{F}$-disks from $2.6 \mathrm{~cm}$ to $10.0 \mathrm{~cm}$, and the H-disks from $9.5 \mathrm{~cm}$ to $26 \mathrm{~cm}$. The barrel detectors measure primarily $r-\phi$. The strips in the modules run in the axial direction, and if $\mathrm{DS}$, at $\pm 2^{\circ}$ or $90^{\circ}$ relative to the axial direction. The disks primarily measure $r-z$, and also $r-\phi$. The detector covers 


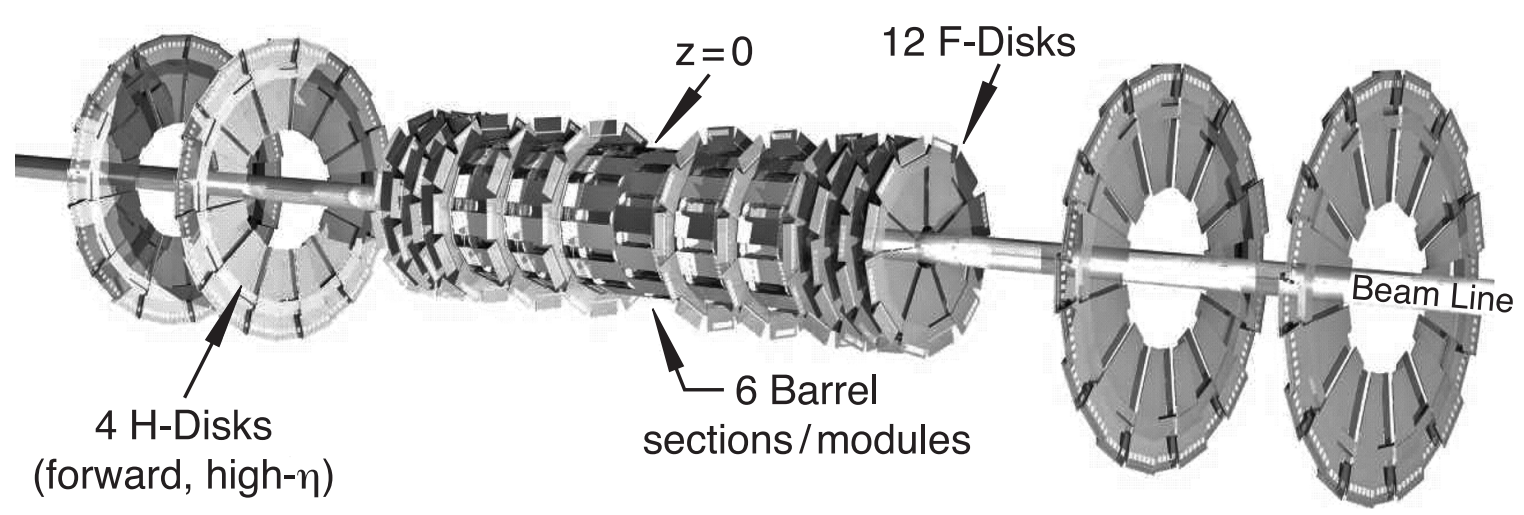

Figure 4.4: The silicon microstrip tracker [39].

$|\eta|<3$. For the barrel modules, the axial hit resolution is around $10 \mu \mathrm{m}$, and the $z$ hit resolution is approximately $35 \mu \mathrm{m}$ for the $90^{\circ}$ stereo, $450 \mu \mathrm{m}$ for the $2^{\circ}$ stereo.

In total there are $793 \mathrm{k}$ channels in the SMT, which are read out using 128-channel SVXIIe chips. These chips include a preamplifier, a 32-cell analog pipeline, and an analog to digital converter (ADC) with a sparse readout [39],.

\section{Central Fiber Tracker}

Surrounding the SMT is the CFT, consisting of 76,800 scintillating fibers. The fibers are arranged in 8 concentric carbon-fiber barrels with radii from 20 to $51 \mathrm{~cm}$. The outer six barrels are $2.52 \mathrm{~m}$ long, while the inner two barrels are only $1.67 \mathrm{~m}$ long, in order to accommodate the silicon H-disks. The coverage of the outer layer is $|\eta|<1.7$. The fibers are arranged in single-layer ribbons, each 128 fibers wide. These singlet layers are then joined to make doublet layers by placing the fiber centers on one layer in the spaces between the fibers of the other layer. The resolution of a fiber doublet is about $100 \mu \mathrm{m}$ [41]. Two doublet layers of fibers are positioned on each of the barrels. The layer closest to the barrel is aligned with the $z$-axis and is called an axial layer. 


\begin{tabular}{|llcccl|}
\hline Module & Type & Layer & Pitch $(\mu \mathrm{m})$ & Angle $\left(^{\circ}\right)$ & Length $(\mathrm{cm})$ \\
\hline F-disk & DS & - & $p: 50, n: 62.5$ & 30 & 7.93 \\
H-disk & SS & - & 40,80 readout & $15^{a}$ & 7.63 inner \\
& & - & & & 6.33 outer \\
Central Barrels (4) & DSDM $^{b}$ & 1,3 & $p: 50, n: 153.5$ & 90 & 12.0 \\
& DS & 2,4 & $p: 50, n: 62.5$ & 2 & $6.0 \times 2^{c}$ \\
Outer Barrels (2) & SS & 1,3 & 50 & - & $6.0 \times 2$ \\
& DS & 2,4 & $p: 50, n: 62.5$ & 2 & $6.0 \times 2$ \\
\hline
\end{tabular}

Table 4.1: SMT sensor specifications.

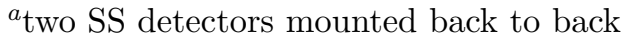

${ }^{b}$ Double Sided, Double Metal

${ }^{c}$ two $6 \mathrm{~cm}$ sensors bonded together

The next layer is aligned with about $\pm 3^{\circ}$ offset with the beam axis. These are called stereo layers or $u$ and $v$ layers. The $u$ and $v$ layers alternate barrel by barrel such that there are eight axial, four $u$, and four $v$ layers in the CFT. Since the CFT covers more radial distance than the SMT, the CFT is better for determining the $p_{\mathrm{T}}$ and charge of charged particles by measuring the curvature of the tracks in the solenoidal magnetic field.

The scintillating fibers are $835 \mu \mathrm{m}$ in diameter. The fiber core is made of polystyrene (PS), doped $1 \%$ by weight with the organic fluorescent dye paraterphenyl (pT). Ionizing particles generally excite the PS, which transfers the excitations to the pT, which in a few nanoseconds has a fluorescent decay, giving off light with a wavelength of $340 \mathrm{~nm}$. Light at that wavelength, however, has a mean free path of only a few hundred microns, so additionally, 3-hydroxyflavone (3HF) is added at $1500 \mathrm{ppm}$ as a wavelength shifter (WLS). The final radiation has a wavelength of $530 \mathrm{~nm}$. Around the core are two thin layers of cladding, the inner made from acrylic, the outer from fluoro-acrylic material. Doubly-clad fibers are used to increase the attenuation length, 
and transmit the light more efficiently than single-clad fibers. At one end of each fiber, there is an aluminum mirror coating to reflect the light. At the other end, a clear waveguide fiber is matched to each scintillating fiber to transport the light from the CFT. The waveguides are identical to the scintillating fibers except that they do not contain the fluorescent dye.

The waveguides carry the light 7 to 12 meters to the readout platform. There the waveguides are connected to cassettes, which are set in a liquid helium cryostat. The light goes through the cassettes to the Visible Light Photon Counters (VLPCs). The VLPCs are small silicon devices with arrays of photo-sensitive areas which convert the light from the fibers to electrical pulses for read-out. The VLPCs operate at about $9 \mathrm{~K}$, have a quantum efficiency of greater than $80 \%$ and have a gain of 20,000 to 50,000 , so a signal that consists of only a few photons can be detected.

\section{Solenoidal Magnet}

The SMT and the CFT are both inside a $2 \mathrm{~T}$ magnetic field provided by the superconducting solenoidal magnet. The magnet's size, $1.42 \mathrm{~m}$ in diameter, $2.73 \mathrm{~m}$ in length, was set by the size of the tracking cavity determined by the calorimeter cryostats, which are from Run I. The magnet has a thickness of $0.9 X_{0}$ at $\eta=0$, where $X_{0}$ is known as a radiation length, or the length needed for an electron's energy to be reduced to $1 / e$ of its original energy due to radiation losses.

The solenoid is wound in two layers of superconducting conductor made of $\mathrm{Cu}: \mathrm{NbTi}$ strands stabilized with aluminum. There are two types of conductors in order to make the current density greater at the ends of the solenoid, which results in better field uniformity. The coils are kept at a superconducting temperature by liquid helium.

The nominal current that flows through the solenoid is $4749 \mathrm{~A}$. After the shutdown 
that took place in 2005 , however, the solenoid could no longer hold such a high current due to resistive heating at a solder joint, so the current had to be reduced to $4550 \mathrm{~A}$. Therefore, after the shutdown, the nominal magnetic field was reduced to $1.92 \mathrm{~T}$. The direction of the current can be reversed so that the magnet can operate in both polarities.

\subsubsection{Preshower Detectors}

To compensate for the material in front of the calorimeters, especially in the solenoidal magnet, preshower detectors were installed outside of the central tracking system. Their purpose is to aid electron and photon identification, both by providing extra tracking to match tracks with calorimeter showers, as well as providing an energy measurement early in the shower development. There are two preshower detectors: the central preshower (CPS), located between the solenoid and the central calorimeter and covering $|\eta|<1.3$, and the forward preshower (FPS), located in front of the end cap calorimeters and covering $1.5<|\eta|<2$.4. Their positions are shown on the diagram of the inner detector, Figure 4.3.

The active layers of the preshower detectors are made from scintillating strips of triangular cross section, as shown in Figure 4.5. The strips are made of PS plastic doped with $1 \% \mathrm{pT}$ and $150 \mathrm{ppm}$ diphenyl stilbene, and they are wrapped in aluminized mylar for optical isolation. In the middle of each strip there is a WLS fiber to collect and transmit the signal. These fibers are polished and silvered at one end, and read out on the other end using the same hardware as the CFT.

The CPS consists of three concentric cylindrical scintillator layers placed behind a $1 X_{0}$ lead radiator, and has 7680 channels of readout. One layer is in the axial direction, and two stereo layers are arranged at $\pm 22.5^{\circ}$. Together, the solenoid and 


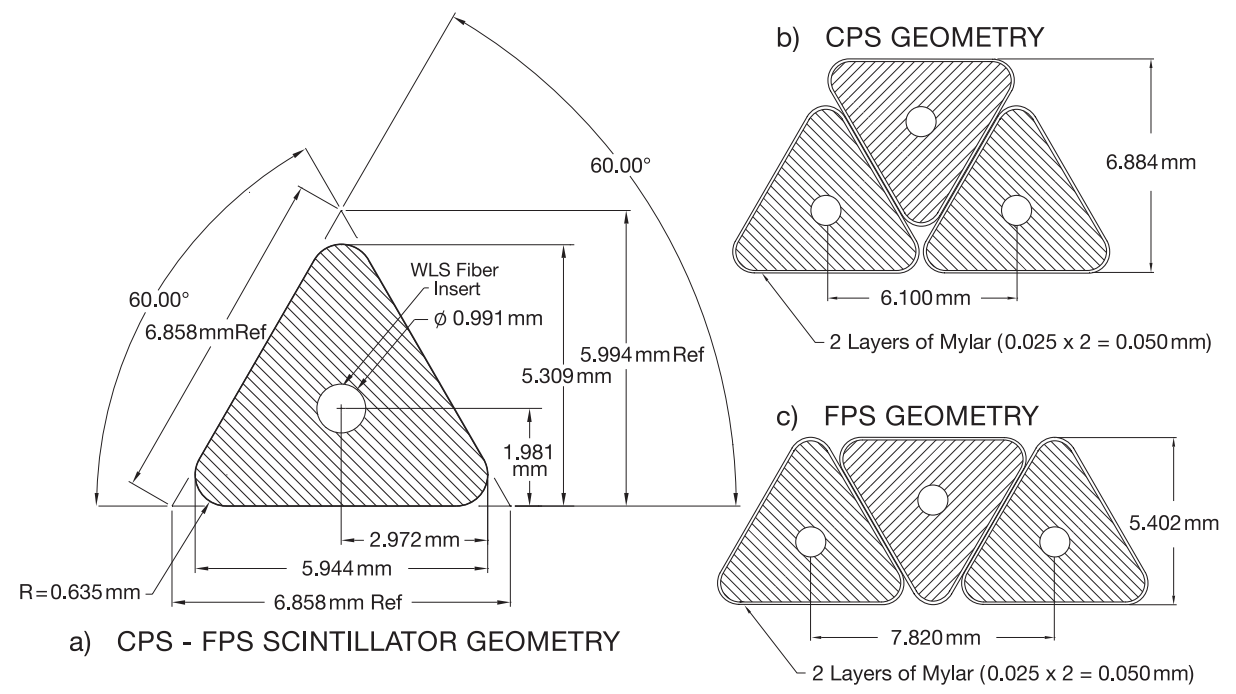

Figure 4.5: Cross-section of the scintillator strips used in the preshower detectors, with layout geometry shown for the CPS and FPS [39].

the lead radiator amount to $2 X_{0}$. At that point electrons and photons should have started showering but other particles should not have thereby providing for some discrimination.

The FPS consists of two similar scintillator-strip detectors mounted on the spherical heads of the North and South endcap calorimeter cryostats, between the intercryostat detector and the luminosity monitor. Figure 4.6 shows a photograph of one of the FPS detectors.

Each FPS detector is made of two active layers, located at different values of $z$. The so-called "MIP layer" is positioned closest to the interaction point (i.e. at smallest $|z|)$. As the name suggests, charged particles traversing the fiducial volume of the detector should provide minimum ionizing particle (MIP) signals in the FPS MIP layer. The MIP layer is followed by an inactive $2 X_{0}$-thick lead absorber. Both the MIP layer and the lead absorber cover the region $1.65<|\eta|<2.5$. The absorber 


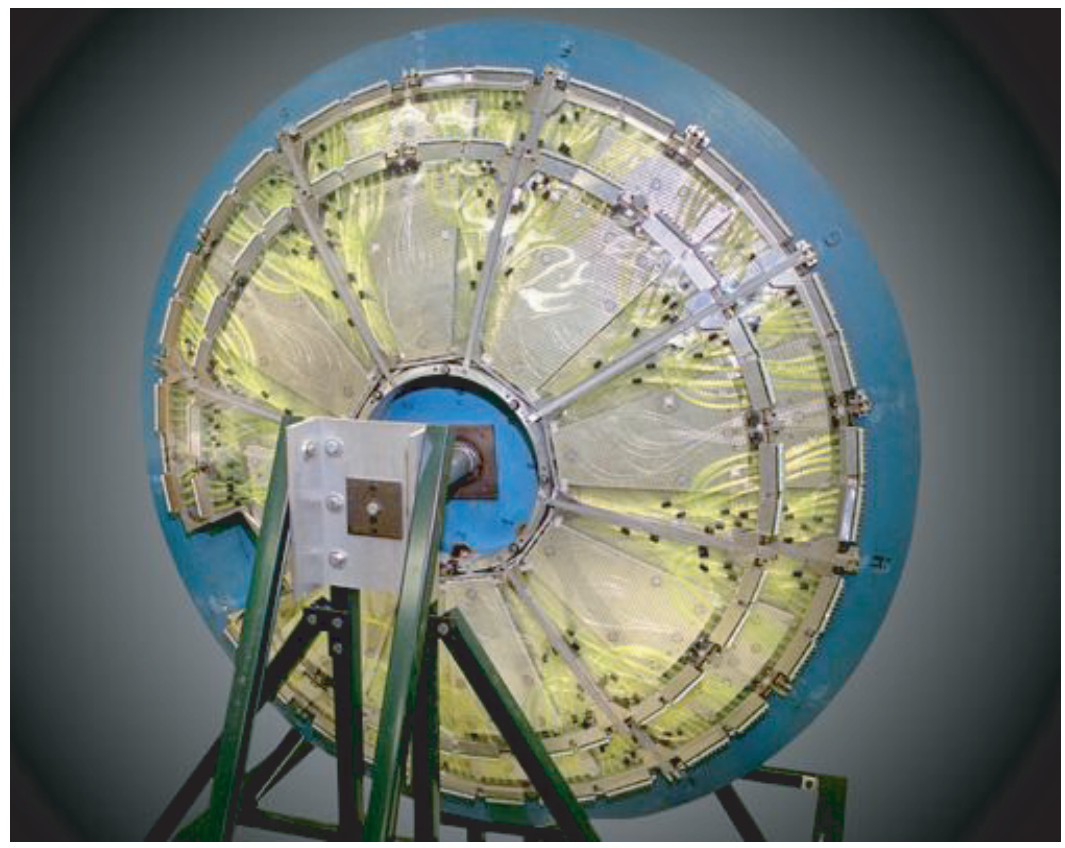

Figure 4.6: Photograph of one FPS detector [44].

is followed by the FPS "Shower layer", so named since it is expected to detect multiMIP signals for electrons (and photons) whose electromagnetic showers have been initiated in the lead absorber. Other particles, on the other hand, would be expected to resemble a MIP also in the Shower layer. The Shower layer covers the region 1.5 $<|\eta|<2.5$.

Clusters for electrons, muons and $\pi^{ \pm}$in the Shower layer should match to hits in the MIP layer in the regions where they overlap. The outer region of the FPS Shower layer $(1.5<|\eta|<1.65)$, where there is no corresponding MIP layer and absorber coverage, lies behind the solenoid coil. The solenoid provides up to $3 X_{0}$ of material in front of the FPS Shower layer, so the lead absorber is not required to initiate electromagnetic showers.

Each FPS layer consists of sixteen wedges of $22.5^{\circ}$ in azimuth. Neighboring FPS 
wedges are offset in order to prevent projective cracks in $\phi$. Each wedge consists of two sub-layers (dubbed $\mathrm{U}$ and $\mathrm{V}$ sub-layers) of nested scintillator strips with a stereo angle of $22.5^{\circ}$ with respect to one another. Each MIP layer wedge contains 206 scintillator strips $(103 \mathrm{U}+103 \mathrm{~V})$, and each Shower layer wedge contains 288 strips $(144 \mathrm{U}+144 \mathrm{~V})$. In the vicinity of the solenoid cryogenics, there are four special wedges that contain 142 scintillator strips $(71 \mathrm{U}+71 \mathrm{~V})$ each. In this area the coverage of the FPS is reduced to $1.8<|\eta|<2.5$.

The FPS readout system shares the same hardware as used for the CFT and CPS, and has been designed to distinguish and measure both minimum ionizing tracks and electromagnetic showers. Light generated by the passage of charged particles through the scintillators is routed through clear fiber waveguides (and bundled on groups of 16 channels) to Visible Light Photon Counter cassettes (VLPC cassettes) where it is converted into an electrical signal [45]. Each VLPC receives input from 8 consecutive scintillating strips. Electrical signals from the VLPCs go to SVX chips mounted on Analog Front-End Boards (AFEs) for digitization.

The initial design of the FPS readout system consisted of 12 multi-chip modules (MCM) mounted on an AFE12 board. The use of 12 MCMs per board would allow studying physics signals over a broad energy range. The gain per MCM could be set so as to trigger on low $\mathrm{p}_{T}$ physics, such as $b$-physics and certain SUSY channels, as well as provide a trigger for high $\mathrm{p}_{T}$ events such as from Higgs, $W$, and top. Instead, for technical reasons, AFE8 boards containing 8 MCMs instead of 12 were used. The use of AFE8 boards prevented the dual threshold setting possible with AFE12 boards, and consequently resulted in a limited dynamic range. As a result, the dynamic range is not adequate to cover higher $p_{T}$ events, thus leading to saturation effects. The loss of information due to the energies deposited in the saturated strips is so severe that 
the unsaturated FPS energy does not correlate strongly with the calorimeter energy. Therefore, the recorded FPS energy cannot be used for Run IIa to correct for energy losses upstream of the endcap calorimeters. The usefulness of the FPS during this running period is, therefore, limited to its use as an additional tracking device in the forward region [44].

\subsubsection{Calorimeters}

The function of a calorimeter is to measure the energy of particles by inducing electromagnetic and hadronic showers. When traversing through material, high energy $(\gg 10 \mathrm{MeV})$ electrons and photons lose their energy through ionization and bremsstrahlung. Above a critical energy $E_{C}$ [46], bremsstrahlung is the dominant process. The critical energy is given by:

$$
E_{C} \simeq \frac{800 \mathrm{MeV}}{Z+1.2}
$$

where $\mathrm{Z}$ is the atomic number of the material. An electromagnetic shower begins when, in the presence of material, an electron radiates a photon through bremsstrahlung. The photon converts into an electron-positron pair, both of which can radiate photons again in turn. As this process repeats itself, a single high energy electron 'converts' into a shower of many lower energy particles traveling in the direction of the initial incident electron. The shower continues till $E_{C}$ is reached. At this point, electrons start losing energy more by ionization, and photons do not have enough energy to convert into electron-positron pairs.

One radiation length, $X_{0}$, is the length for an electron to have its energy reduced by a factor of $1 / e$ due to radiation losses only. Additionally, it is found that the mean 
path photons travel before pair-producing electrons is approximately $\frac{9}{7} X_{0}[46]$. For electromagnetic calorimeters, the material thickness is expressed in terms of radiation lengths. As an example, the radiation length $X_{0}$ for uranium is about $3.2 \mathrm{~mm}[7]$.

Charged particles, other than electrons, with energies that are typical of processes at the Tevatron, lose energy in matter primarily by ionization and atomic excitation. The energy loss per unit length is given by the Bethe-Bloch equation [46]:

$$
-\frac{d E}{d x}=K z^{2} \frac{Z}{A} \frac{1}{\beta^{2}} \frac{1}{2}\left[\ln \frac{2 m_{e} c^{2} \beta^{2} \gamma^{2} T_{\max }}{I^{2}}-\beta^{2}-\frac{\delta}{2}\right]
$$

where $K=4 \pi N_{A} r_{e}^{2} m_{e} c^{2}, A$ is the atomic mass of the absorbing material, $\beta=\frac{v}{c}, Z$ is the atomic number of the absorbing material, $T_{\max }$ is the maximum kinetic energy that can be imparted to a free electron in a single collision, $I$ is the mean excitation energy of the atoms in the absorbing material, $\delta$ is a density effect correction to the ionization loss, $r_{e}$ is the classical radius of an electron, $N_{A}$ is Avogadro's number, $z$ is the charge of incident particle in units of $e, m_{e}$ is the mass of electron, $c$ is the speed of light, and $\gamma=\frac{1}{\sqrt{1-\beta^{2}}}$.

Hadronic particles which enter the calorimeter can interact inelastically with the nuclei of the absorbing layers. The interactions produce mostly pions and nucleons that can collide inelastically with other nuclei. Thus, a hadronic shower is initiated when a high energy hadron enters the calorimeter. The mean path between inelastic interactions ("nuclear interaction length") $\left(\lambda_{A}\right)$, provides a scale for the shower development, and is roughly independent of energy and depends on the density and the weight of the material. It is given by [47]:

$$
\lambda_{A} \simeq 35 \mathrm{gcm}^{2} A^{1 / 3}
$$


where $\mathrm{A}$ in the atomic weight of the material. For uranium, $\lambda_{A} \simeq 10.5 \mathrm{~cm}[7]$. Therefore, hadronic showers develop over a longer distance than electromagnetic showers, especially for high atomic number $(Z)$ materials.

The only SM particles that do not shower in the calorimeters are muons and neutrinos. Muons leave MIP signals in the calorimeter and there is a dedicated detector for them, covered in Section 4.2.5, while neutrinos are only implicitly observed by conservation of momentum.

One of the strengths of the original Run I D0 detector was the high quality of its calorimeters. Therefore, though the readout was changed in order to handle the shorter time between bunch crossings, the calorimeters themselves are largely unchanged. The calorimeters are shown in Figure 4.7.

The D0 calorimeters are segmented into cells. Figure 4.8 shows a schematic of a calorimeter cell. Each cell consists of layers of absorbing material, to induce shower formation, and active layers where atoms are ionized by the passage of charged particles. A calorimeter that has alternating absorption and active layers is called a "sampling calorimeter". Depleted uranium², copper and stainless steel are the absorbers used, while liquid argon (LAr) serves as the active medium. In the uranium of the absorber plates, the radiation length and the interaction length is short, so the particles can be stopped in a relatively short distance. However, they are "dead material" in the sense that no signal is read from them. Instead, there are the gaps between the absorber plates filled with LAr where the shower is sampled, hence the name. The LAr is ionized by charged particles passing through it, and the charges are collected on the copper pads of the signal boards that are located in each cell. In this way the total energy of the incident particle or jet can be measured by sum-

\footnotetext{
${ }^{2}$ Depleted uranium has the uranium-235 isotope depleted.
} 


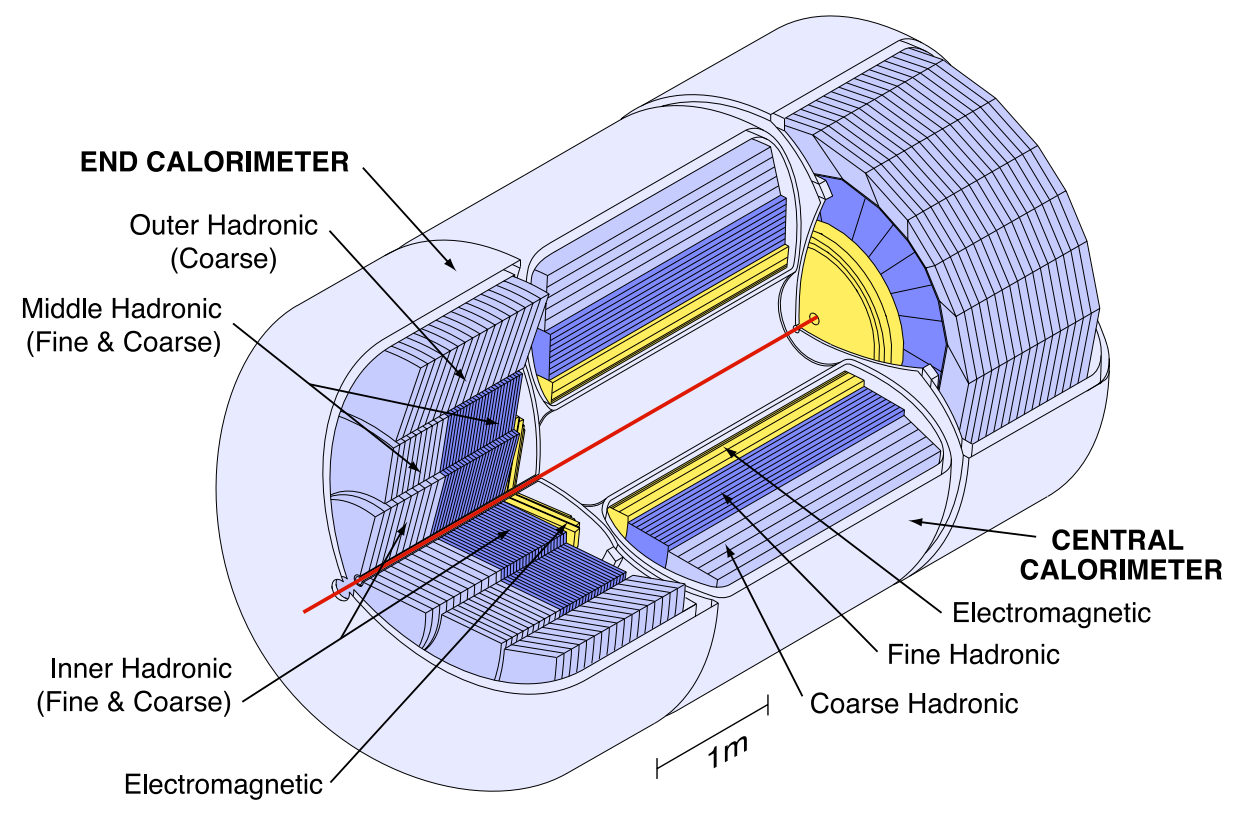

Figure 4.7: The D0 calorimeters and their modules [39].

ming the charge collected in all of the cells through which it passes. Calibration of the calorimeter is essential for the observed charged to be converted into an energy measurement.

Typically, in calorimetry, the fraction of energy deposited by an electron or photon is greater than the energy deposited by a hadron because much of the low-energy hadronic component is absorbed in nuclear binding energy release, etc. and hence is not detected. The use of depleted uranium makes the D0 calorimeter a compensating one, meaning that the calorimeter response to electromagnetic and hadronic activity is equal (or in other words $\frac{e}{h}=1$ ). Low energy neutrons, from the nuclear breakup in hadronic showers, cause fission in the uranium and energy is converted into charged particles by the $\beta$ decay of the fission products. This compensation is a useful feature because a hadronic shower contains an electromagnetic component from $\pi^{0}$ decays and 


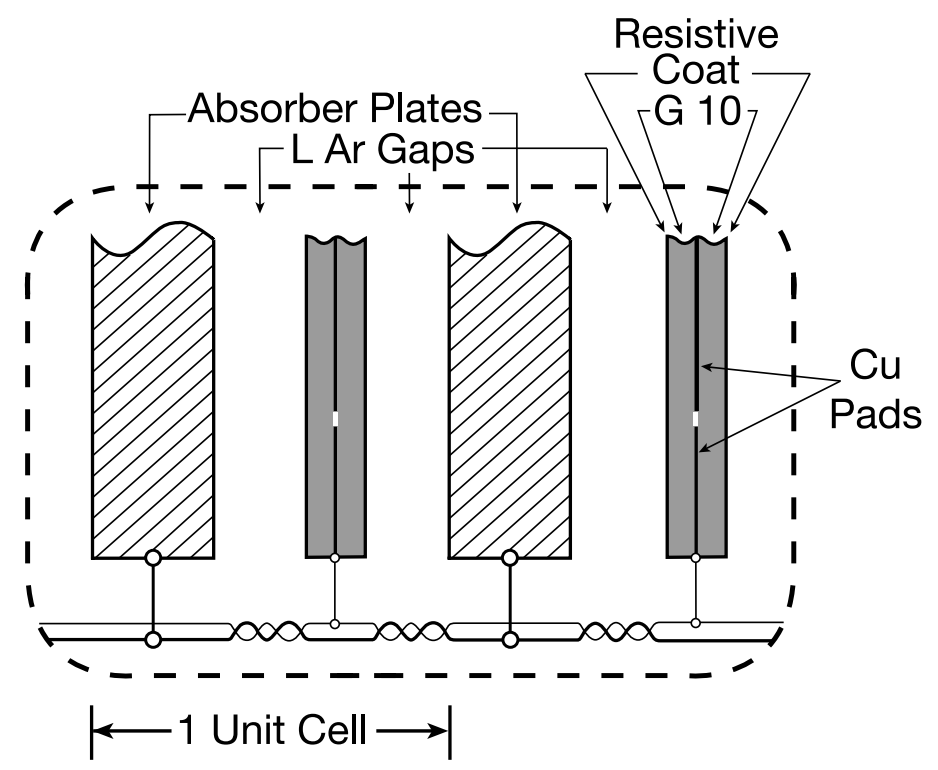

Figure 4.8: Schematic view of a Calorimeter Cell [39].

hence a non-compensating calorimeter suffers from problems related to an energy dependent response ratio for electrons and hadrons, and a non-linear response to hadrons. The performance of a non-compensating calorimeter is compromised owing to fluctuations in the $\pi^{0}$ content of the showers and this results in a skewed signal distribution, and an almost constant contribution to the energy resolution which is proportional to the degree of non-compensation $\left(1-\frac{h}{e}\right)$. For the D0 calorimeter the ratio of the electromagnetic and hadronic response is close to one and ranges from 1.11 at $10 \mathrm{GeV}$ to 1.04 at $150 \mathrm{GeV}$ [38].

The D0 calorimeters are housed in 3 large cryostats, one in the central region $\left(\left|\eta_{\text {det }}\right|<1.2\right)$ and one in each endcap, extending the coverage up to $\left|\eta_{\text {det }}\right| \simeq 4.5$. The central calorimeter (CC) weighs about 330 tons, whereas each of the endcap calorimeters (ECS, ECN, or collectively EC) weighs about 240 tons.

Closest to the interaction point are the electromagnetic (EM) sections of the 
calorimeters, whose purpose is to accurately measure the energies of electrons and photons. They are built with $3 \mathrm{~mm}$ (CC) or $4 \mathrm{~mm}$ (EC) thick uranium plates. The EM modules are divided into four depth layers (EM1, EM2, EM3, and EM4), known as "floors", of approximately 1.4, 2.0, 6.8, and $9.8 X_{0}$ thick in the CC, and 1.6, 2.6, 7.9 , and $9.3 X_{0}$ thick in the EC [39]. The values for the first floor include the material in the cryostat wall.

Next in depth are the hadronic sections. In the CC, the hadronic modules are divided into fine hadronic $(\mathrm{FH})$ modules which use $6 \mathrm{~mm}$ thick uranium-niobium $(2 \%)$ alloy plates, and one coarse hadronic $(\mathrm{CH})$ module which uses $46.5 \mathrm{~mm}$ thick plates of copper. In turn, the FH modules are divided into three floors (FH1, FH2, FH3) of depth $1.3,1.0$, and $0.76 \lambda_{A}$. The lone $\mathrm{CH}$ floor is approximately $3.2 \lambda_{A}$ thick. Some important design parameters for the CC are summarized in Table 4.2.

In the EC, the hadronic modules are divided into the inner hadronic (IH), the middle hadronic $(\mathrm{MH})$, and the outer hadronic $(\mathrm{OH})$. The $\mathrm{IH}$ and $\mathrm{MH}$ are additionally split into fine and coarse sections. For IH the finer section consists of four floors (FH1, FH2, FH3, and FH4), each $1.1 \lambda_{A}$ thick. The coarse hadronic section has a single floor $4.1 \lambda_{A}$ thick. Similarly, for the MH the finer section consists of four floors (FH1, FH2, FH3, and FH4), each $0.9 \lambda_{A}$ thick. The coarse hadronic section has a single floor $4.4 \lambda_{A}$ thick. The $\mathrm{OH}$ modules are made of stainless steel plates inclined at an angle of about $60^{\circ}$ with respect to the beam axis(see Figure 4.9). There are three floors with a maximum thickness of $6.0 \lambda_{A}$. A summary of some important design parameters for the EC are listed in Table 4.3.

Most of the floors, except EM3, are segmented transversely into cells of $\Delta \eta=0.1$ and $\Delta \phi=2 \pi / 64 \approx 0.1$. In EM3 the shower maximum is expected to occur ${ }^{3}$, so

\footnotetext{
${ }^{3}$ In Run I the peak of electromagnetic showers occurred in EM3. In Run II, with the
} 


\begin{tabular}{|l|c|c|c|}
\hline Module Type & $\mathrm{EM}$ & $\mathrm{FH}$ & $\mathrm{CH}$ \\
\hline Rapidity Coverage & \pm 1.2 & \pm 1.0 & \pm 0.6 \\
Number of Modules & 32 & 16 & 16 \\
Absorber & $\mathrm{Ur}$ & $\mathrm{Ur}-\mathrm{Nb}$ & $\mathrm{Cu}$ \\
Absorber Thickness (mm) & 3 & 6 & 46.5 \\
Argon Gap (mm) & 2.3 & 2.3 & 2.3 \\
Total Radiation Lengths & 20.5 & 96.0 & 3.2 \\
Total Nuclear Radiation Lengths & 0.76 & 3.2 & 3.2 \\
\hline
\end{tabular}

Table 4.2: Parameters of the Central Calorimeter(CC).

\begin{tabular}{|l|c|c|c|c|c|c|}
\hline Module Type & EM & IFH & ICH & MFH & MCH & OH \\
\hline Number of Modules & 1 & 1 & 1 & 16 & 16 & 16 \\
Absorber & Ur & Ur-Nb & SS & Ur-Nb & SS & SS \\
Absorber Thickness (mm) & 4 & 6 & 46.5 & 6 & 46.5 & 46.5 \\
Argon Gap (mm) & 2.3 & 2.1 & 2.1 & 2.2 & 2.2 & 2.2 \\
Total Radiation Lengths & 21.4 & 121.8 & 32.8 & 115.5 & 37.9 & 65.1 \\
Total Nuclear Radiation Lengths & 0.97 & 4.4 & 4.1 & 3.6 & 4.4 & 6.0 \\
\hline
\end{tabular}

Table 4.3: Parameters of the End-Cap Calorimeter(EC).

in order to achieve better precision in determining the position of electromagnetic showers, a finer resolution was chosen. In EM3 the segmentation is $\Delta \eta=0.05$ and $\Delta \phi=2 \pi / 128 \approx 0.05$. The cells are arranged into pseudo-projective towers, as shown in Figure 4.9. A feature of the D0 calorimeter that must be taken into account is the intermodule cracks in the coverage in the $\mathrm{CC}$, the so-called $\phi$-cracks. The energy of electromagnetic showers in that area can be mismeasured ${ }^{4}$. The effect of the $\phi$-cracks on the analysis will be discussed in more detail in Chapter 6 .

addition of the solenoid and preshower detectors, there is an additional 2-3 $X_{0}$ in front of the calorimeter. Thus the shower peak occurs earlier, in the front part of the EM3.

${ }^{4}$ Later detectors, like ATLAS at LHC, avoid that issue by using an accordion shape design. 


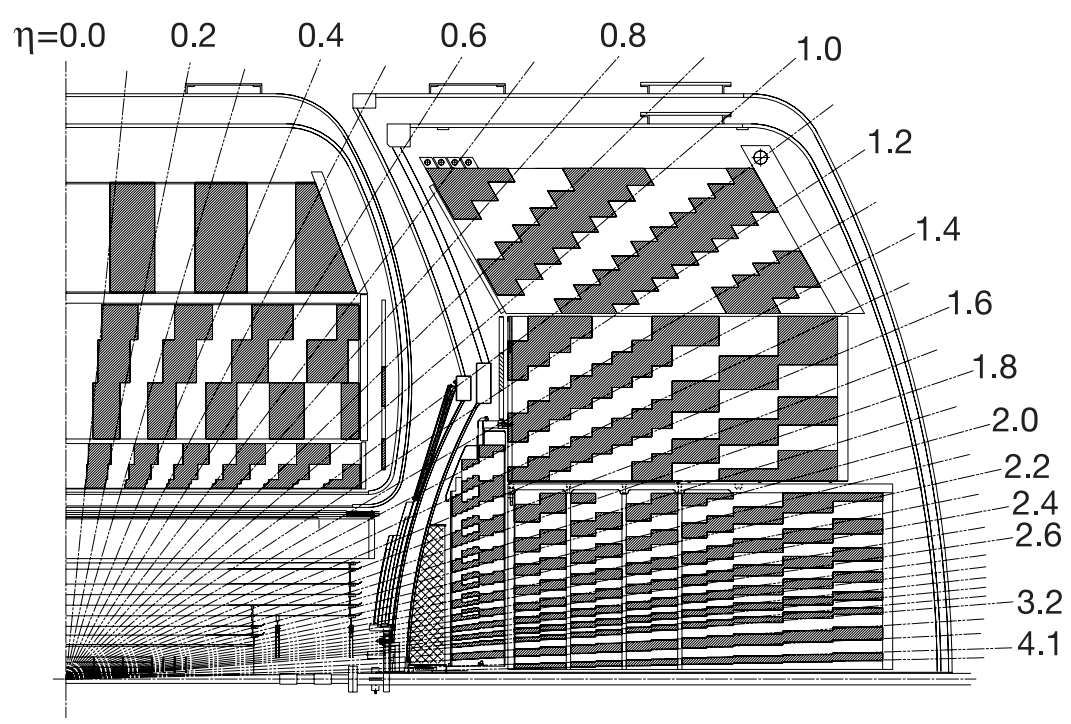

Figure 4.9: Schematic view of the calorimeter showing the transverse and longitudinal segmentation pattern [39].

As can be seen in Figure 4.9, in the area between the CC and the EC cryostats, there are certain trajectories that pass through much material before hitting the actual calorimeter structures. In order to add sampling to those trajectories, the massless gaps (MG) have been added within the cryostats, and the intercryostat detector (ICD) has been added between the cryostats. Those detectors can be seen in Figure 4.9, though they have not been labeled in order to not over-complicate the figure. The ICD is also visible and labeled in Figure 4.3. The massless gaps are standard calorimeter cells in front of the first layer of uranium in both the CC and the EC. The ICD which covers $1.1<|\eta|<1.4$, is a series of 0.5 in thick Bicron BCF-400 scintillating tiles. The tiles are divided into subtiles of $\Delta \eta \times \Delta \phi \approx 0.1 \times 0.1$ to match the calorimeter. The subtiles are read out by WLS fibers, which via clear fibers are sent to photo-multiplier tubes (PMTs). The signal is shaped and made to 
be compatible with the standard calorimeter signals.

The calorimeter readout chain is shown in Figure 4.10. A charge proportional to the energy loss of the particles traversing the cell is sent to the readout electronics through four ports in the cryostats via $30 \Omega$ coaxial cables (with a typical length of $10 \mathrm{~m})$. First, the charge is integrated in the preamplifier to produce a voltage. Then, the voltage pulses are carried by twist and flat cables to the shaper and baseline subtracters (BLS), which shape the signal and remove slowly varying offsets in the input voltage. The shaped signal is sampled at its peak at about $320 \mathrm{~ns}$. Because the LAr drift time is about $450 \mathrm{~ns}$, only $2 / 3$ of the charge in the calorimeter is actually used. The shaped signals are stored in switched capacitor arrays (SCAs) until a Level 1 trigger decision is made $(\sim 4 \mu s)$. If a positive decision is made, the signal is sent to a second SCA buffer to await a Level 2 trigger decision $(\sim 100 \mu s)$. Finally, the output signal is digitized by the Analog to Digital Converters (ADCs) and sent to the data acquisition system (DAQ). The readout system is designed to have no dead-time up to a Level 1 trigger rate of $10 \mathrm{kHz}$, assuming one interaction per bunch crossing [39].

The relative momentum resolution for the calorimeter system is measured in data and found to be $\sigma\left(p_{\mathrm{T}}\right) / p_{\mathrm{T}} \approx 0.13$ for $50 \mathrm{GeV}$ jets in the $\mathrm{CC}$ and $\sigma\left(p_{\mathrm{T}}\right) / p_{\mathrm{T}} \approx 0.12$ for $50 \mathrm{GeV}$ jets in the ECs. The energy resolution for electrons in the $\mathrm{CC}$ is $\sigma(E) / E \approx$ $0.16 / \sqrt{E} \oplus 0.04$, where $\mathrm{E}$ is measured in $\mathrm{GeV}$ and $\oplus$ means addition in quadrature.

\subsubsection{Muon System}

The only directly detectable particles that are able to pass through the calorimeter are high energy muons. The muons pass through the calorimeter as minimum ionizing particles (losing around $1.6 \mathrm{GeV}$ of energy), so they need to be identified by the muon system [39], which in addition measures their locations, and their transverse momenta. 


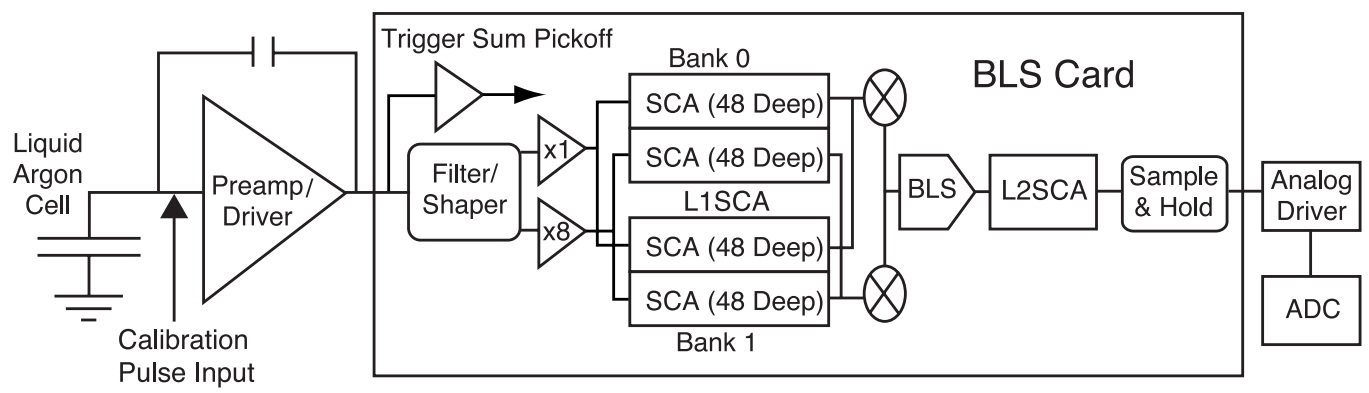

Figure 4.10: Readout chain of the calorimeter in Run II. Major components are: preamplifiers, BLS, and the ADC [39].

The muon system consists mainly from three components:

- Central detector; Wide angle muon spectrometer (WAMUS) that covers $|\eta|<1$

- Forward detector; Forward angle muon spectrometer (FAMUS) that covers $1<$ $|\eta|<2$

- Solid-iron magnet generating toroidal field of $1.8 \mathrm{~T}$.

The WAMUS consists of three layers of proportional drift tubes (PDTs) and two layers of scintillator plates with embedded WLS fibers. The purpose of the scintillators is to have a fast readout for accurate timing, rejecting cosmics, matching wire chamber hits with bunch crossings, and triggering. There are no scintillators in the middle layer. The FAMUS consists of three layers each of mini-drift tubes (MDTs) and scintillator pixels. Figure 4.11 shows the exploded view of the arrangement of the wire chambers and scintillators. Note that the bottom of the detector is not fully instrumented.

Since muons and the muon system are not used in this analysis, there is no need for further details. More information can be found in [39]. 

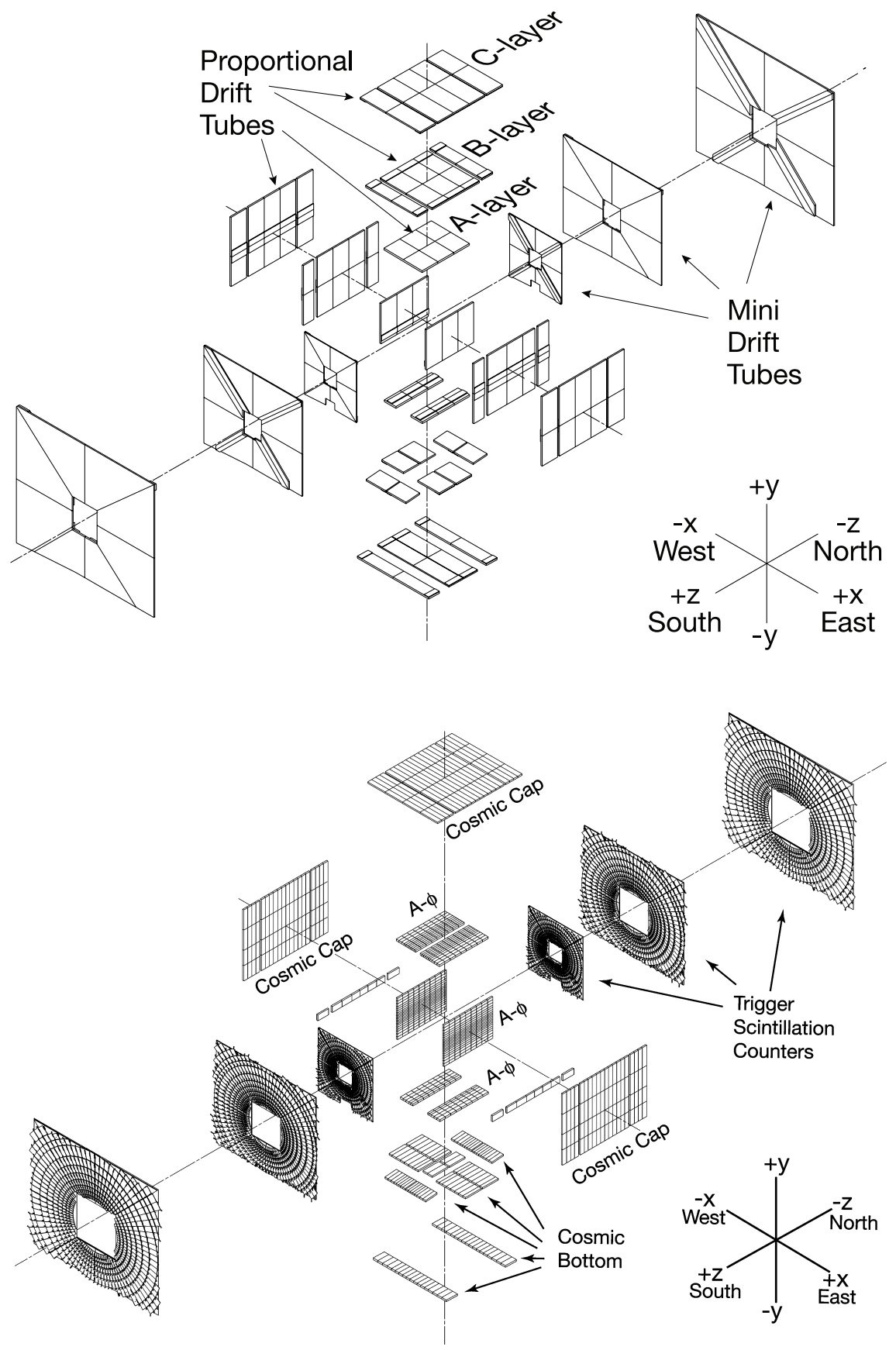

Figure 4.11: Exploded view of the muon system wire chambers (top) and scintillators (bottom) [39]. 


\subsubsection{Luminosity Monitor}

The main purpose of the luminosity monitor (LM) is to measure the luminosity that the Tevatron delivers at the $\mathrm{D} 0$ interaction region. This is accomplished by detecting inelastic $p \bar{p}$ collisions.

The LM detector consists of two arrays of 24 plastic scintillator counters with PMT readout. They cover $2.7<|\eta|<4.4$, are placed in front of the EC Calorimeters, and occupy the radial region between the beam pipe and the FPS at $z \sim \pm 140 \mathrm{~cm}$. Figure 4.12 shows the location of the LM detectors.

The instantaneous luminosity $\mathcal{L}$ is determined from the average number of inelastic collisions per beam crossing $\bar{N}_{\mathrm{LM}}$ measured by the LM. The instantaneous luminosity is a measure of the particle flux per unit area per unit time $\left(\mathrm{cm}^{-2} \mathrm{~s}^{-1}\right)$ and is defined as:

$$
\mathcal{L}=f \bar{N}_{\mathrm{LM}} / \sigma_{\mathrm{LM}}
$$

where $f$ is the beam crossing frequency $\left(2.53 \mathrm{MHz}\right.$ or $\frac{1}{396 \mathrm{~ns}}$, and $\sigma_{\mathrm{LM}}$ is the effective cross section, taking into account the acceptance and efficiency of the LM [48]. Assuming that particles hitting the LM detector originate from a $p \bar{p}$ interaction, the $z$ coordinate of the interaction vertex $z_{v}$ can be estimated by the difference in time-offlight:

$$
z_{v}=\frac{c}{2}\left(t_{-}-t_{+}\right)
$$

where $t_{+}$and $t_{-}$are the time-of-flight measured for particles hitting the LM detectors placed at $\pm 140 \mathrm{~cm}$. Collisions are selected by requiring $\left|z_{v}\right|<100 \mathrm{~cm}$, that practically includes all the $p \bar{p}$ collisions produced by Tevatron $\left(\sigma_{z} \approx 25 \mathrm{~cm}\right)$, and distinguish from $p \bar{p}$ interactions from the beam halo background. Beam halo particles that travel in the $\pm \hat{z}$ direction have $\left|z_{v}\right| \approx \mp 140 \mathrm{~cm}$ and fail the $\left|z_{v}\right|<100 \mathrm{~cm}$ cut. 


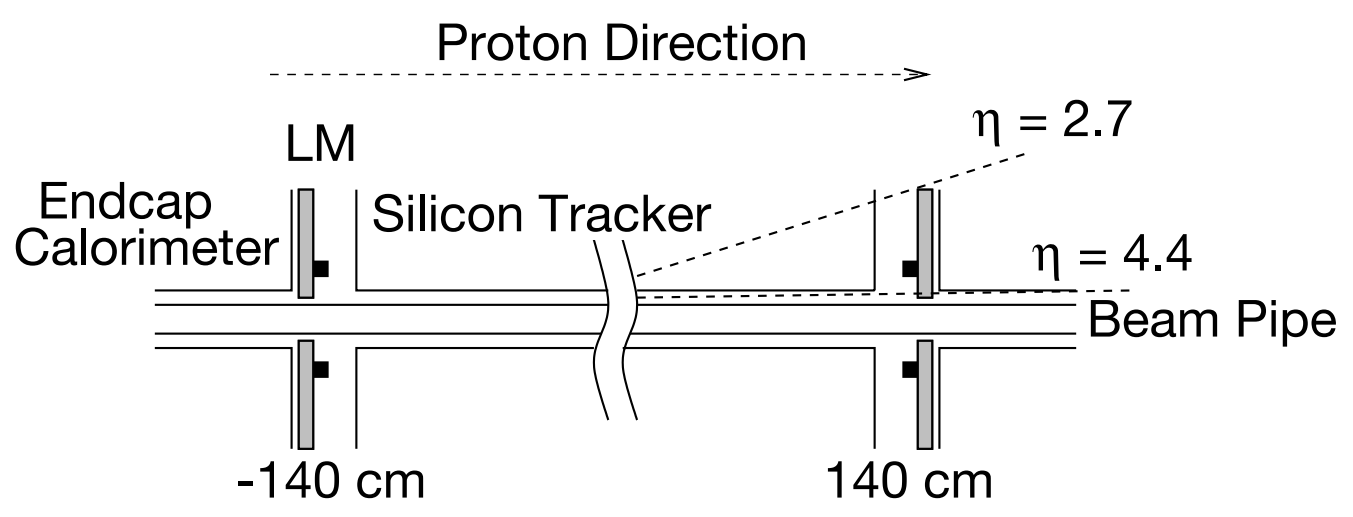

Figure 4.12: Schematic drawing showing the location of the Luminosity Monitor (LM) detectors [39].

The fundamental unit of time used for the luminosity measurement is called the luminosity block (LB). Each block is indexed by the luminosity block number (LBN), which continuously increases throughout Run II. The LBN is incremented upon run or store transitions, trigger framework (see Section 4.3) or SCL initialization, by request, or after $60 \mathrm{sec}$ have elapsed. The time period is selected to be short enough so that the instantaneous luminosity is practically constant during each luminosity block. Thus, negligible uncertainty is introduced into the measurement of luminosity due to the width of the time interval. The uncertainty on the luminosity is currently estimated to be $6.1 \%$ [49].

\subsection{The D0 Trigger and Data Acquisition Systems}

Bunch crossings take place at the Tevatron every $396 \mathrm{~ns}$, that is at a rate of $\sim 2.5 \mathrm{MHz}$. However, as discussed previously in Section 4.1, the beam is structured into superbunches with $2 \mu$ s between each super-bunch, and bunches with $396 \mathrm{~ns}$ between each 
bunch inside a super-bunch. Consequently, the collision rate at the D0 detector is reduced to $\sim 1.7 \mathrm{MHz}$ by the presence of the $2 \mu$ s gaps in the beam. Even at this rate it is not technically feasible to read out and store all the data. One can reliably store to tape up to a rate of $\sim 30 \mathrm{MB} / \mathrm{s}$. Most of the collisions at the Tevatron are small angle inelastic collisions that have already been well studied. Interesting high energy, hard interactions occur at much smaller rates. A real-time decision of which events are "interesting" enough to be stored must be made. This process uses information from the various sub-systems in order to reduce the incoming rate from $\sim 1.7 \mathrm{MHz}$ to $\sim 50 \mathrm{~Hz}$.

D0 uses a three-level triggering system, with each level reducing the rate into the next level. An overview of the trigger and data acquisition (DAQ) system is shown in Figure 4.13. The Level 1 (L1) trigger system reduces the rate by a factor of 1000 . The Level 2 (L2) trigger system reduces the rate by an additional factor of 2 , and Level 3 (L3) reduces the rate by a further factor of 10 . At the beam crossing rate of $\sim 1.7 \mathrm{MHz}$, detector information is passed to the L1 trigger. The L1 trigger system has at most $4 \mu$ s of processing time per event to reach a decision. It is a hardwarebased system due to the high input data rate it handles and the tight timing latency requirement.

As an overview of the system, once L1 condition is satisfied, the trigger framework (TFW) sends a L1 accept to the detector sub-systems, and the detector information is sent to L2. The L2 trigger system is based on both special hardware and embedded microcontrollers. Since the L2 trigger system has more time $(\sim 100 \mu s)$ to spend than the L1 trigger on the events, it can implement more sophisticated algorithms and make more correlations between the detectors. Figure 4.14 shows the individual components of the first two trigger levels. Once the L2 trigger system generates a 


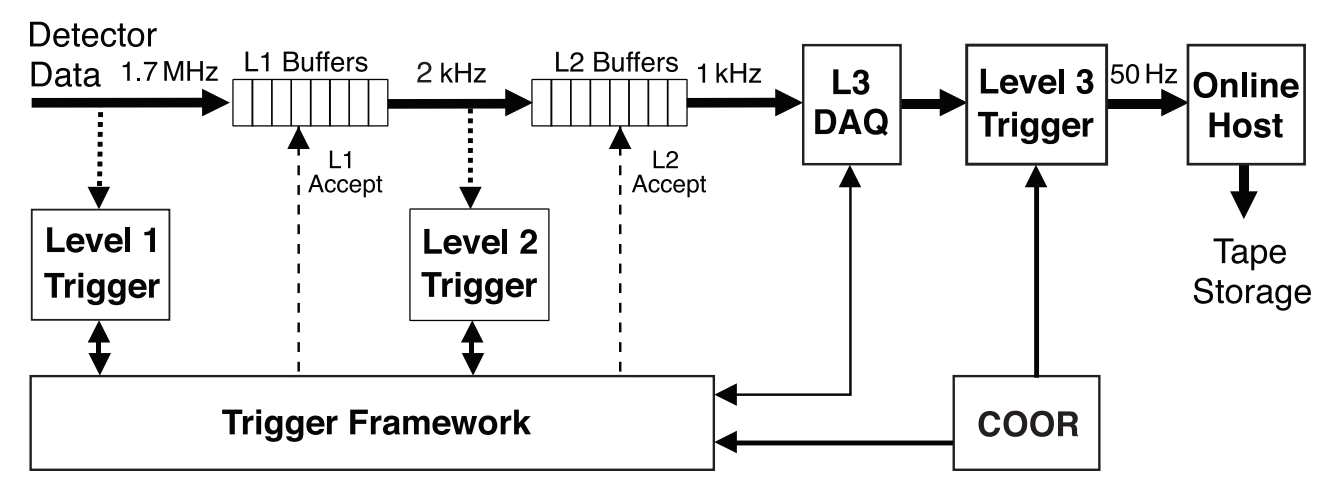

Figure 4.13: Overview of the trigger and DAQ systems [39].

decision, that decision is passed to the TFW. The TFW sends a L2 accept to the detector sub-systems which send the data to L3. The L3 trigger is software-based, running on a computer farm, with access to the full information of the events and does partial reconstruction of the events with algorithms similar to those used offline. The data acquisition system (DAQ) is responsible for the coordination of the L3 farm nodes and the online run control. At that stage the trigger has $150 \mathrm{~ms}$ to reach a decision. The accepted events are distributed by the online host for offline reconstruction (write to tape) and monitoring purposes. The rate has been reduced to $\sim 50 \mathrm{~Hz}^{5}$. The overall coordination and control of D0 triggering and data acquisition is handled by the COOR software package running on the online host.

\subsubsection{Level 1 Trigger}

The L1 trigger generates decisions using information from all detector sub-systems except for the SMT. All events awaiting L1 trigger decisions are pipelined and thus

\footnotetext{
${ }^{5}$ Currently extra capacity has been added and the accept rate can increase to $100 \mathrm{~Hz}$ during high instantaneous luminosity times at the beginning of a store.
} 


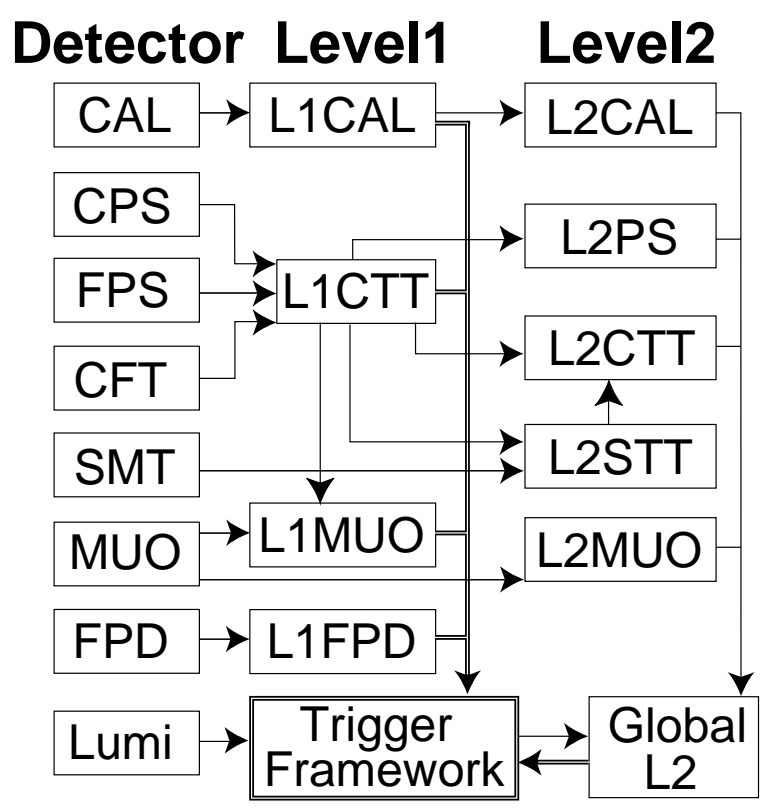

Figure 4.14: Overview of the L1 and L2 trigger systems [39].

make minimal contributions to the deadtime. As mentioned earlier, in order to participate in the trigger decision, the L1 trigger decision must arrive at the TFW in $3.5 \mu \mathrm{s}$ or less. The rate of $\mathrm{L} 1$ trigger accepts is limited by the maximum readout rates of the participating subsystems and by a desire to minimize the deadtime associated with the readout. The digitized readout systems have enough memory to hold 32 bunch crossings.

\section{L1 Calorimeter Trigger}

The L1 calorimeter (L1CAL) trigger adds up the energy, distinguishing only EM and FH layers in depth, in towers with lateral segmentation of $\Delta \eta \times \Delta \phi \approx 0.2 \times 0.2$. These $0.2 \times 0.2$ towers are called trigger towers. There are forty trigger towers in $\eta$ covering $|\eta|<4$.0. Each slice in $\eta$ has thirty-two trigger towers covering the full $2 \pi$ 
of the azimuth $(\phi)$. During Run IIa, whose data are used for this analysis, the Level 1 triggers are only defined in terms of the numbers of trigger towers with $E_{\mathrm{T}}$ above thresholds, measured either only in the EM layer or in the full trigger tower $(\mathrm{EM}+$ $\mathrm{FH})$.

\section{L1 Central Track Trigger}

The L1 central track trigger (L1CTT) reconstructs trajectories of charged particles using axial hits from the CFT and the PS detectors. The CFT and CPS axial system provide triggers for charged particles using predefined track equations and matching tracks to PS clusters. L1CTT conditions can be specified by the number of tracks above a $p_{\mathrm{T}}$ threshold, with or without a PS cluster match, and track isolation.

\section{L1 Muon Trigger}

The L1 Muon trigger (L1Muon) uses hits from the muon wire chambers, muon scintillator counters, and tracks from L1CTT to form patterns consistent with muons. L1Muon forms trigger objects based on L1CTT tracks and muon scintillator hits. L1Muon also forms trigger objects based on matching layers of track stub (group of interlayer hits) wire hits which have been confirmed with muon scintillators. L1Muon conditions can be specified by the number of muons above a $p_{\mathrm{T}}$ threshold, geographical region, and track quality.

\section{Trigger Framework}

The TFW makes the decision whether a particular event is to be accepted for further examination by using the inputs of the L1 trigger devices. The TFW uses the logical 
"OR" of up to 128 specific triggers conditions defined by the trigger list to determine if a given crossing holds a valid trigger. The TFW also manages the rates of triggers by applying prescale factors to keep their rates within acceptable limits. Different trigger lists and prescale settings are passed by COOR using the trigger control computer (TCC). The TFW provides a large number of scalars to monitor trigger rates and dead times.

\subsubsection{Level 2 Trigger}

The L2 trigger system was designed to operate within a $\sim 100 \mu$ s time window and to reduce the L1 rate by a factor of 10. During physics data taking, the L2 trigger typically receives events at a rate of $1.5 \mathrm{kHz}$ and has a rejection factor from two to five.

The L2 trigger consists of five detector-specific preprocessing engines and a global stage (L2Global) that test for correlations in physics signatures across detector subsystems. L2 preprocessors collect data from the front-ends and L1 trigger system and analyze these data to form physics objects. The L2Global combines them to form the final L2 trigger decision. L2GBL can have up to 128 separate trigger decisions to process before accepting or rejecting an event. If the event passes at least one trigger, then this event is passed along to the L3 trigger system for further review.

\section{L2 Calorimeter Preprocessor}

The L2 calorimeter preprocessor (L2CAL) system identifies jets and EM objects and calculates event $\mathbb{E}_{T}$. L2CAL receives a list of $1280 \mathrm{EM}$ and $1280 \mathrm{EM}+\mathrm{HAD}$ trigger towers from L1CAL to form the EM and jet objects. The EM algorithm forms electron 
and photon objects using EM trigger towers. A cluster is formed by a seed trigger tower with $E_{T}>1 \mathrm{GeV}$ and its largest $E_{\mathrm{T}}$ neighboring EM tower. EM fraction and isolation fraction are calculated for each EM object. The jet algorithm forms jet objects by clustering $5 \times 5 \mathrm{EM}+\mathrm{HAD}(3 \times 3$ before trigger version v9.31 $)$ trigger tower clusters, centered around a seed tower with $E_{\mathrm{T}}$ greater than $2 \mathrm{GeV}$ [50]. The $\mathrm{EM}$ and jet objects are independently sorted in descending order of clustered $E_{\mathrm{T}}$ and sent to L2Global.

\section{L2 Silicon Track Trigger Preprocessor}

The L2 silicon track trigger preprocessor (L2STT) uses L1CTT tracks and SMT hits to improve the momentum resolution of the tracks and to calculate track impact parameters. The impact parameter calculation from L2STT is used to tag the decays of long-lived particles such as B hadrons. The L2STT algorithm fits track parameters by projecting $\mathrm{L} 1 \mathrm{CTT}$ tracks into the SMT detector. The fitted tracks are sent to L2CTT.

\section{L2 Central Track Trigger Preprocessor}

The L2 Central Track Trigger preprocessor (L2CTT) sends tracks to L2Global to match tracks to physics objects. L1CTT tracks are combined and sorted in descending order of $p_{\mathrm{T}}$. The azimuthal angle, with respect to the beam axis is determined, as well as the azimuthal angle of the track projected to the third EM layer of the calorimeter. Several isolation criteria are calculated to enhance the trigger capabilities. The L2CTT processor sends three lists of tracks to L2Global: a $p_{\mathrm{T}}$ sorted list of L1CTT tracks, a $p_{\mathrm{T}}$ sorted list of L2STT tracks, and an impact parameter sorted list of L2STT tracks. 


\section{L2 Preshower Preprocessor}

The L2 Preshower preprocessor (L2PS) is used to improve the electron detection efficiency and photon separation. All three layers of the PS detectors are used to form clusters in $\eta$ and $\phi$. L2PS also reports L1CTT tracks that match with PS clusters.

\section{L2 Muon Preprocessor}

The L2 Muon preprocessor (L2Muon) combines track segments among small regions and layers of the detectors in preprocessing units called Second Level Input Computers (SLICs). The sub layers are combined into integrated muon candidates in the preprocessor. The muon candidates are sorted in descending order of $\mathrm{pT}$ and are sent to L2Global. The quality and timing information per muon candidate are also passed to L2Global.

\section{L2 Global Processor}

L2Global is the first level of the trigger to examine correlations across all the detector systems. L2Global receives trigger objects from the L2 preprocessors. Trigger decisions are made by creating global physics objects. These objects can be based directly on the objects reported by the preprocessors or can be created by combining objects from different preprocessors. L2Global uses the trigger list and the L1 trigger decision mask to decide which script to run on the objects. Each script is defined by at least one or more filters and a minimum number of objects required to pass each filter. An example of a script is an EM object filter and a minimum of two objects. This script is satisfied if there are two EM objects in the event that satisfied the 
conditions of the filter. If any script is satisfied, the event passes L2 and is sent to L3.

\subsubsection{Level 3 Trigger and Data Acquisition}

At the final stage of the trigger system is a dedicated computer farm that performs a fast reconstruction using a simpler version of the offline reconstruction code (described in Chapter 5). The final trigger decision is made on high level "physics" objects (such as electrons, muons, and jets) as well as on the relationships between such objects (such as the azimuthal angle separating the objects or their invariant mass). The system's designed bandwidth is $250 \mathrm{MB} / \mathrm{s}$, and this corresponds to an average event size of approximately $200 \mathrm{kB}$ at an L2 Accept rate of $1 \mathrm{kHz}$.

Following a L2 Accept, the data for that event is transferred out from each of the readout crates by a Single Board Computer or SBC (sitting in each crate) via a large ethernet switch. The data is sent to one or more farm nodes specified by routing instructions received from the routing master (RM) process running on an SBC in a special crate containing a hardware interface to the TFW.

A program running on the farm nodes runs the event reconstruction and an event filter. At first software algorithms called "physics tools" are used to generate candidate objects and the relations between them. Individual calls to the tools are made by "filter scripts". A filter script contains a list of the physics tools that are to be used to process the event, and the parameters (defined by filters) to be passed

to the tools. A limited number of parameter sets (called reference sets or refsets) are used for each physics tool (e.g. three refsets are used to define an electron, each with different selection criteria). An event is passed by the trigger if all the filters for any 
of the filter scripts pass. These accepted events are written to tape for offline analysis and status information indicating which scripts passed or failed is passed along as well. 


\section{Chapter 5}

\section{Offline Event Reconstruction and Object Identification}

Raw detector data that is measured by the D0 detector and is stored on tape needs to be processed and combined to create physics objects, to be used in physics analyses. The offline reconstruction is accomplished by the reconstruction package d0reco [51, $52]$, that is a collection of complex software algorithms.

The reconstruction is performed, sequentially, in the following steps:

- Detector specific processing

- Detector data blocks are unpacked

- Raw information is decoded

- Readout channels are associated with physical detector elements

- Calibration constants are applied

- Pre-reconstruction 
- Cluster reconstruction for the calorimeter and preshower detectors

- Hit reconstruction for the tracking systems

- Tracking

- Reconstruction of global tracks from hits in the SMT and CFT incorporating different algorithms

- Vertexing

- Reconstruction of primary vertex candidates

- Identification of displaced secondary vertices

- Particle Identification

- Reconstruction of physics objects: electrons, photons, muons, and jets

- Identification of heavy-quark ( $b$ and $c$ ) jets, as well as $\tau$ candidates

- Reconstruction of missing transverse energy $\mathscr{E}_{T}$

Details of these steps that are most relevant to this dissertation are discussed in the following subsections

\section{$5.1 \quad$ Tracking}

A typical event in the central tracking system contains $10^{4}$ to $10^{6}$ hits. Charged particles can deposit energy to two adjacent silicon strips or two adjacent scintillating fibers, so the hits are clustered together. These clusters are used as input to the track fitting algorithms to find tracks. There are two track finding algorithms that are used 
in D0: the Histogramming Track Finder (HTF) [53], and the Alternative Algorithm (AA) [54].

The HTF method uses the fact that in the $(r, \phi)$ plane, the charged particles in a magnetic field travel in circular orbits uniquely defined by the curvature of the track $\rho=\frac{q B}{p_{\mathrm{T}}}$, the distance of closest approach (dca) $d_{0}$ to the beam spot, and the direction $\phi$ of the track at the position of the closest approach to the beam spot. Every pair of hits in coordinate space $(x, y)$, corresponds to a single point in parameter space $(\rho, \phi)$. Given minimal $p_{\mathrm{T}}$ the parameter space can be divided into cells thus creating a 2D histogram. All hits forming a track have multiple pair combinations. All pair combination will have the same value (bin) in the $(\rho, \phi)$ histogram. The pattern recognition (track hypothesis) is made by taking a single hit in $(x, y)$ and extrapolating it to a line in the $(\rho, \phi)$ histogram. This mapping of points to lines in parameter space is called a Hough transformation. All hits from the same track will have separate lines which will all intersect at the same bin, the true $(\rho, \phi)$ of the track in question.

The track list is passed to a 2D Kalman filter which uses $\rho, d_{0}$, and $\phi$ of each track, an expectation propagator, material effects (multiple scattering and energy loss), and the non-uniformity of the magnetic field to filter the track list [55, 56]. The remaining tracks pass through another histogramming algorithm that uses the hit locations $(r, z)$ to form lines in $\left(z_{0}, C\right)$ coordinate space, where $z_{0}$ is the starting location of the track along the $z$ axis, and $C=d z / d r$. The lines which overlap in the $\left(z_{0}, C\right)$ space generate a reduced track list. The list is processed through an $\eta$ splitter, that only allows hits moving away from the interaction point to be associated with a track when the $z$ component of the hits are increasing for $\eta>0$, and similarly when the $z$ component of the hits are decreasing for $\eta<0$. 
Then a 3D Kalman filter is used to build the SMT tracks and continues including hits in the CFT detector until there are too many misses in a row or the algorithm reaches to the end of the detector. Beginning with the partially reconstructed track, the 3D Kalman filter extends the track by an additional measurement or hit. The track parameters and the expectation propagator are used to make an expected hit measurement. A $\chi^{2}$ is calculated and if the value is under the maximum allowed limit, the hit is accepted. The track parameters are recalculated for this track, and the algorithm is repeated searching for the next hit. An additional HTF list is generated by starting from the CFT instead of the SMT using the same techniques. The two track lists are combined and duplicate tracks are removed.

The alternate algorithm (AA) [54] starts in 2D with three SMT hits for pattern recognition and then applies a track filter algorithm. The first hit can come from any of the six layers in the silicon barrels or F disk. The second hit must be on a following layer within $\Delta \phi$ of 0.08 rads. The third hit should be on a following layer, within a circle of radius greater than $30 \mathrm{~cm}$ and axial impact parameter with the beam spot of less than $2.5 \mathrm{~cm}$. The overall fit must have $\chi^{2}<16$. Each track is extrapolated to the next layer of the SMT or CFT repeatedly, and hits are added to the track hypothesis if the increase of $\chi^{2}$ is less than 16. If there are multiple hits in a given layer, they each become new hypotheses. A certain number of misses (i.e., no hits) in layers are allowed to improve the efficiency. The fitting algorithm continues until it has three consecutive misses in a row, or it reaches the end of the detector. The track hypotheses are ordered based on number of hits, and those that have equal number of hits by the fewest number of misses, and those that have the same number of hits and misses, by the better the $\chi^{2}$ of the fit. Since the fitted tracks may share hits from other tracks, the AA requires that the number of hits shared to be less than $2 / 3$ of the total number of hits in the track. To further reduce the number of fake 
tracks, primary vertices are determined using the accepted tracks, and every track that comes close to a vertex is given two additional hits in the rankings. Using this new weight, the tracks are resorted, and a new pool of tracks is determined.

The description so far would preclude tracks with no SMT hits. Therefore, the same procedure is repeated starting with three CFT hits, but to control the huge combinatorics with stereo hit associations, the tracks must pass near a primary vertex determined from the SMT tracks.

The two track lists from the HTF and the AA algorithms are combined into a single list, refitted, and smoothed, using the Kalman algorithm [56].

\subsection{Primary Vertices}

The location of the hard scatter is known as the primary vertex (PV). The $x$ and $y$ locations of the PV fluctuates within $40 \mu \mathrm{m}(1 \sigma)$ between events. The z location of the PV is roughly a Gaussian distribution with a spread $(\sigma)$ of $\sim 30 \mathrm{~cm}$. The main difficulty determining the PV is distinguishing which tracks come from it and which come from secondary vertices due to heavy quark decays or additional minimum bias interactions that are incidentally close to the PV. To accomplish this, an adaptive PV algorithm [57] is used.

As a first step, the algorithm clusters tracks with $p_{\mathrm{T}}>0.5 \mathrm{GeV}$ and two or more SMT hits if they are in an area with SMT acceptance, into different interaction areas $2 \mathrm{~cm}$ long along the $z$ axis. Tracks with the highest $\chi^{2}$ contribution are removed, 
until the total vertex $\chi^{2}$ per degree of freedom is smaller than 10 . Subsequently only tracks with $\left(\mathrm{dca} / \sigma_{\mathrm{dca}}<5\right)$ are used, where $\sigma_{\mathrm{dca}}$ is the standard deviation of the dca distribution.

Then, the adaptive primary vertex algorithm, assigns weights $w_{i}$, for each track $i$. The weights $w_{i}$ are calculated applying the following function:

$$
w_{i}=\frac{1}{1+e^{\left(\chi_{i}^{2}-\chi_{\text {cutoff }}^{2}\right) / 2 T}}
$$

where $\chi_{i}^{2}$ is the $\chi^{2}$ contribution of each track $i$ to the vertex, and tunable parameters $\chi_{\text {cutoff }}^{2}$ and $T$ receive the values 4 and 1 , respectively. The algorithm:

1. Starts with all weights $w_{i}=1$.

2. Weighting each track by its weight $w_{i}$, determines the primary vertex using a Kalman Filter.

3. Updates the weights $w_{i}$ using the new vertex. If $w_{i}<10^{-6}$, sets the weight to zero for that particular track.

4. If all the weights changed by less than $10^{-4}$, stops. Otherwise, repeats from step 2. (There is also a maximum number of iterations exit in case convergence fails.)

Finally, the primary vertex of the hard scatter must be chosen, while the other vertices are expected to come from minimum bias events. Minimum bias events have low $p_{\mathrm{T}}$ tracks, so a probability can be assigned to each track, based on its $p_{\mathrm{T}}$, as to whether it comes from a minimum bias interaction [58]. For each vertex, the track probabilities are multiplied together and weighted so that the final probability value 
does not depend on the number of tracks associated with the vertex. The vertex chosen as the primary vertex is the one with the lowest such probability.

This algorithm has been shown to be very robust. As an example, for $t \bar{t} \rightarrow e+$ jets the resolution is $8.4 \pm 0.3 \mu \mathrm{m}$ with a pull of $1.07 \pm 0.02$, and for $t \bar{t} \rightarrow e \mu$ the resolution is $10.3 \pm 0.6 \mu \mathrm{m}$ with a pull of $1.12 \pm 0.03$ [59].

\subsection{Calorimeter Preprocessing}

Before using the calorimeter data to reconstruct objects, the data is processed to remove unphysical energy deposits and noise. During data taking, individual cells can show high energy due to hardware problems. Such cells are called "hot", and are usually suppressed so as not to affect data taking rates. At the L3 trigger and in the early stages of offline processing, the New Anomalous Deposits Algorithm (NADA) is used to further suppress hot cells and transient spikes in energy [60,61, 62]. The principle behind NADA is that if a cell has a high energy deposit $\left(E_{\text {cell }}>1 \mathrm{GeV}\right.$ in the original algorithm) while the sum of the energy of the 26 cells that surround it in a $3 \times 3 \times 3$ cube is low $\left(E_{\text {cube }}<100 \mathrm{MeV}\right.$ in the original algorithm, but only summing the energy of cells in the cube with $E>100 \mathrm{MeV}$ ), then the energy deposit is deemed unphysical, and the cell's energy is set to $1 \mathrm{MeV}$. There are special cases for certain layers. such as EM3, and at calorimeter boundaries in depth or $\eta$, and some of the thresholds can be dynamic, scaled as a fraction of the cell energy for energetic cells, but the basic idea is the same.

A similar algorithm is used to remove noise; the T42 algorithm [63, 64, 65]. The noise source could be from electronics, uranium decays, pile-up from interactions in previous bunch crossings, etc. Zero-suppression zeroes all cells with absolute energies 
less than $2.5 \sigma$ above the pedestal value to remove most of this noise. The T42 algorithm additionally removes all negative energies. Furthermore, the T42 algorithm zeroes all cells with energy between $2.5 \sigma$ and $4 \sigma$ above the pedestal value unless the cell has a neighbor with an energy over $4 \sigma$ above the pedestal. Thus, around energy deposits that are considered to be signal-like, the zero-suppression is $2.5 \sigma$, but where there is no signal-like deposit, it is raised to $4 \sigma$. Neighbors are chosen similarly as in the NADA algorithm.

Electrons, photons, and jets are reconstructed using the cone algorithm. Energy deposition in a cell is represented by a massless four-vector [66] that points along the cell's direction from the center of the detector. The cone algorithm starts by selecting seeds, starting with the highest $E_{T}$ calorimeter towers in the event above a certain threshold. The cone space is defined as:

$$
\Delta R=\sqrt{\left(\Delta \eta_{\text {det }}\right)^{2}+\left(\Delta \phi_{\text {det }}\right)^{2}}
$$

where $\Delta \eta_{\text {det }}\left(\Delta \phi_{\text {det }}\right)$ is the difference in pseudorapidity (azimuthal angle) between a tower and the seed tower. The 4-vectors of towers within a fixed cone size are added to the seed four-vector. The centroid of the cone is calculated from contributions from all the particles within the cone. The cells within a cone are then removed from consideration, and a new seed is formed from the remaining highest- $E_{\mathrm{T}}$ tower.

\subsection{Electrons}

The electron identification algorithm selects electrons and rejects background. Sources of background are: $\pi^{0}$ showers which overlap with a nearby track, photons that convert to $e^{+} e^{-}$pairs, charged pions, and jet fluctuations. A study showed $\pi^{0}$ or $\eta$ 
mesons to be the most common particles that fake electrons after requiring a track matched to the electron candidate [67].

Electrons start out as calorimeter objects [68, 69]. Electromagnetic particles (electrons, positrons, and photons) are reconstructed using the energy from the four EM layers and the first hadronic layer of the calorimeter, which defines an EM tower. The algorithm uses a cone of $\Delta R<0.4$ in $(\eta, \phi)$ around seed towers with $p_{\mathrm{T}}>$ $500 \mathrm{MeV}$. Using the notation of $E_{\mathrm{EM}}$ being the energy in the EM layers and $E_{\text {tot }}$ the total energy of the cluster, the cluster becomes an electron candidate if it has:

$$
\begin{gathered}
p_{\mathrm{T}}>1.5 \mathrm{GeV} \\
f_{\mathrm{EM}} \equiv \frac{E_{\mathrm{EM}}}{E_{\text {tot }}}>0.9 \\
f_{\text {iso }} \equiv \frac{E_{\text {tot }}(R<0.4)-E_{\mathrm{EM}}(R<0.2)}{E_{\text {tot }}(R<0.4)}<0.2
\end{gathered}
$$

and has at least $40 \%$ of its energy in its most energetic tower. In equation 5.5, $E(R<r)$ refers to the energy (EM or total) within a cone in $(\eta, \phi)$ of radius $r$. Thus, electrons are isolated calorimeter clusters with most of their energy in the EM layers of the calorimeter. Tightening the isolation requirement for the electrons to $f_{\text {iso }}<0.15$ the jet background is further reduced. Figure 5.1 shows plots of $f_{\mathrm{EM}}$ and $f_{\text {iso }}$ for $\mathrm{CC}$ electrons and fakes. For this plot and all others in this section, the real electrons are from dielectron samples dominated by $Z \rightarrow e^{+} e^{-}$events, and the background is from a "fake" electron that arises from QCD multijet events. The algorithm as described above is the loosest electron quality definition used. It is called Preselect_iso15 because it is the preselection step with $f_{\text {iso }}<0.15$. Many other electron quality definitions build upon this one. There is a similar "looser" Preselect_iso20 definition with only the $f_{\text {iso }}<0.2$ cut, that this analysis uses. 

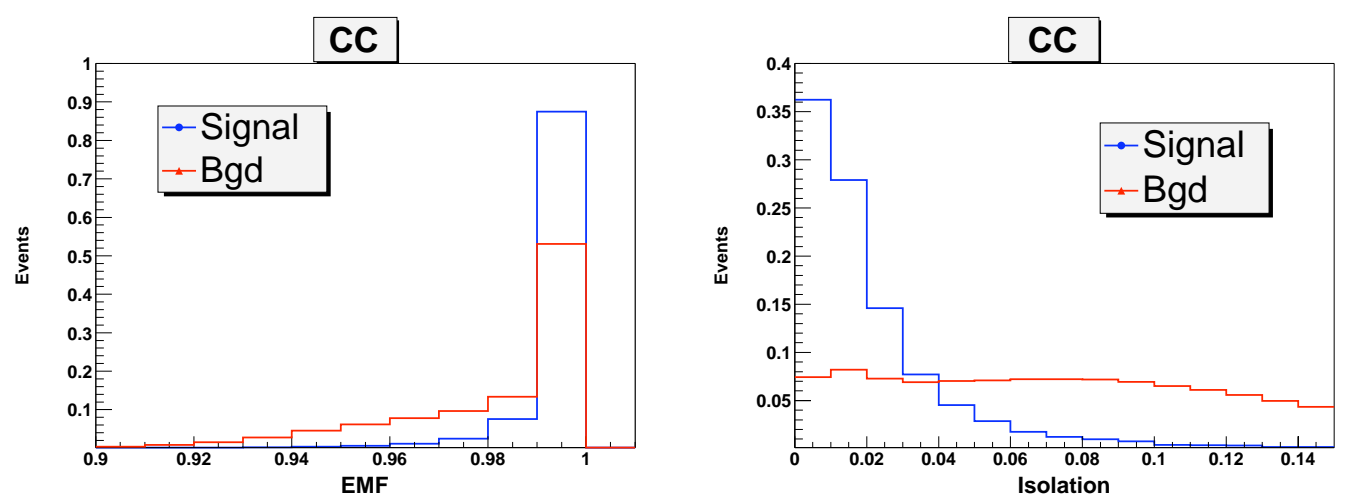

Figure 5.1: The $f_{\mathrm{EM}}$ (left) and $f_{\text {iso }}$ (right) distributions for real and fake electrons [69].

Because the Preselect_iso15 algorithm does not have a track match, it also accepts photons with high efficiency, but the $f_{\mathrm{EM}}$ and $f_{\text {iso }}$ requirements significantly suppress jets. The efficiency of this algorithm in the $\mathrm{CC}$ for real electrons with $p_{\mathrm{T}}>15 \mathrm{GeV}$ from $Z \rightarrow e^{+} e^{-}$events is around $(96 \pm 2) \%$ [70], with the main losses due to intermodule cracks in the coverage in the $\mathrm{CC}$, the " $\phi$-cracks". In the fiducial areas, the efficiency is $(99.2 \pm 0.8) \%$. The efficiency was measured using a tag and probe technique $^{1}$ on an electron+track data set dominated by $Z$ events.

In order to provide more sophisticated and sensitive electron quality definitions, the following variables are introduced:

\section{- H-matrix:}

The purpose of the H-matrix is to measure how compatible the pattern of energy deposits in the calorimeter are with an electron shower [71]. Given N electrons,

\footnotetext{
${ }^{1}$ In the tag and probe technique, one electron candidate - the tag- is required to pass tight cuts to improve the purity of the sample, while the other candidate - the probe- is required to pass the cuts that efficiency is determined. The efficiency $\epsilon$ is then given by $\epsilon=\frac{N_{\text {pass }}}{N_{\text {pass }}+N_{\text {fail }}}$, where $N_{\text {pass }}$ is the total number of probe candidates passing the appropriate cuts and $N_{\text {fail }}$ is the total number failing.
} 
the covariance matrix is calculated as:

$$
M_{i j}=\frac{1}{N} \sum_{n=1}^{N}\left(x_{i}^{(n)}-\bar{x}_{i}\right) H_{i j}\left(x_{j}^{(n)}-\bar{x}_{j}\right)
$$

for a set of electron shower variables, $x_{i}$, and their means, $\bar{x}_{i}$. We then define $H=M^{-1}$. For a given electron candidate $k$, we can determine a $\chi^{2}$ of how well its shower matches that of an electron:

$$
\chi_{h m}^{2}=\sum_{i, j=1}^{7}\left(x_{i}^{(k)}-\bar{x}_{i}\right) H_{i j}\left(x_{j}^{(k)}-\bar{x}_{j}\right)
$$

The lower the value of $\chi_{h m}^{2}$, the more electron-like the shower is. For electrons in the central calorimeter a seven-variable H-matrix variant is used:

1. Energy fraction in the EM1 floor.

2. Energy fraction in the EM2 floor.

3. Energy fraction in the EM3 floor.

4. Energy fraction in the EM4 floor.

5. The $r \phi$ width of the shower in the EM3 floor.

6. $\log _{10}(E)$

7. $z_{\mathrm{vtx}} / \sigma_{z_{\mathrm{vtx}}}$

For electrons in the endcap calorimeter, an eight-variable H-matrix variant is used; the seven mentioned above, with the addition of the $z$ width of the shower in the EM3 floor.

\section{- Track match $\chi^{2}$ :}

Electrons are expected to leave a track in the central tracking system. This 
track should be well centered with the calorimeter cluster. Having a trackmatch provides a good way to distinguish electrons from neutral particles that do not leave a track. Additionally, the quality of the track match can be used to distinguish between electrons and neutral particles that incidentally overlap with a track. The track match $\chi^{2}$ can be defined in two ways:

$$
\begin{aligned}
\chi_{\text {spatial }}^{2} & =\left(\frac{\delta \phi}{\sigma_{\phi}}\right)^{2}+\left(\frac{\delta z}{\sigma_{z}}\right)^{2} \\
\chi_{\text {with } E / p}^{2} & =\left(\frac{\delta \phi}{\sigma_{\phi}}\right)^{2}+\left(\frac{\delta z}{\sigma_{z}}\right)^{2}+\left(\frac{E_{\mathrm{T}} / p_{\mathrm{T}}-1}{\sigma_{E_{\mathrm{T}} / p_{\mathrm{T}}}}\right)^{2}
\end{aligned}
$$

where the variables are defined as

$-\delta \phi=\phi_{\text {track }}-\phi_{\text {clus }}$, where the angles are measured at the EM3 floor. $\sigma_{\phi}$ is the expected width of $\delta \phi$ for an electron.

$-\delta z=z_{\text {track }}-z_{\text {clus }}$, where the $z$ values are measured at the EM3 floor. $\sigma_{z}$ is the expected width of $\delta z$ for an electron.

- $E_{\mathrm{T}}$ is the transverse energy of the cluster.

$-p_{\mathrm{T}}$ is the transverse momentum of the track.

$-\sigma_{E_{\mathrm{T}} / p_{\mathrm{T}}}$ is the expected width of $E_{\mathrm{T}} / p_{\mathrm{T}}$ for an electron.

For electrons $E_{\mathrm{T}} / p_{\mathrm{T}} \approx 1$, but if a track is randomly associated with the calorimeter cluster, this would not necessarily be the case. What is used to define the quality of the track mach is the probability for a track to have a certain $\chi^{2}$, $P\left(\chi^{2}\right)$.

- Likelihood [67]:

A number of variables (noted as the vector $x$ ) can be put together to define a 
likelihood that a track-matched electron object is really an electron:

$$
L=\frac{P_{\mathrm{sig}}(x)}{P_{\mathrm{sig}}(x)+P_{\mathrm{bkg}}(x)}
$$

where the probability for signal $P_{\mathrm{sig}}(x)=\prod_{i} P_{\mathrm{sig}, i}\left(x_{i}\right)$ and background $P_{\mathrm{bkg}}(x)=$ $\prod_{i} P_{\mathrm{bkg}, i}\left(x_{i}\right)$ are the products of the probabilities for the individual variables. The associated track is the one with the highest $P\left(\chi_{\text {spatial }}^{2}\right)$ that has $\delta \phi<0.05$ and $\delta \eta<0.05$. The version of the likelihood that we use is based on seven variables:

1. spatial track match probability, $P\left(\chi_{\text {spatial }}^{2}\right)$

2. (calorimeter cluster $\left.E_{\mathrm{T}}\right) /\left(\operatorname{track} p_{\mathrm{T}}\right)$

3. The distance of closest approach (DCA) of the associated track to the primary vertex

4. H-matrix $\left(\chi_{h m}^{2}\right)$

5. EM fraction $\left(f_{\mathrm{EM}}\right)$

6. The number of tracks in a cone of size $R=0.05$ in $(\eta, \phi)$ around the track

7. The sum of the transverse momenta of all the tracks other than the associated track in a cone of size $R=0.4$

In this case, the spatial part and $E_{\mathrm{T}} / p_{\mathrm{T}}$ are used as two separate variables. Isolated electrons should come from the primary vertex, so the DCA should be small. Electrons are expected to have low $\chi_{h m}^{2}$ values and $f_{\mathrm{EM}} \approx 1$. A loose cut of $\chi_{h m}^{2}<50$ is applied before using the likelihood. Finally, the last two variables are track isolation variables. Electron tracks should be single, clean tracks, while electrons from photon conversion should have two tracks, and jets or parts of jets faking electrons should have extra tracks. 
Plots of selected variables used in the likelihood are given in Figure 5.2.

After the two Preselect definitions, the next tighter electron quality is called loose_notrk. As the name implies there is no track-match requirement. It is defined by:

- Fulfill Preselect_iso20 quality requirements

$$
\begin{aligned}
& -f_{\text {iso }} \leq 0.2 \\
& -f_{\mathrm{EM}} \geq 0.9 \\
& -p_{\mathrm{T}} \geq 3.0
\end{aligned}
$$

- $\mathrm{H}-\operatorname{Matrix}(7) \leq 50$ in the $\mathrm{CC}$

- $\mathrm{H}-\operatorname{Matrix}(8) \leq 75$ in the $\mathrm{EC}$

A plot of the total efficiency for data and MC as a function of $\eta_{\text {det }}$ is given in Figure 5.3.

A tighter version is called tight_notrk, and is defined by:

- Fulfill Preselect_iso20 quality requirements

$$
\begin{aligned}
& -f_{\text {iso }} \leq 0.2 \\
& -f_{\mathrm{EM}} \geq 0.9 \\
& -p_{\mathrm{T}} \geq 3.0
\end{aligned}
$$

- $\mathrm{H}-\operatorname{Matrix}(7) \leq 12$ in the $\mathrm{CC}$

- $\mathrm{H}-\operatorname{Matrix}(8) \leq 20$ in the $\mathrm{EC}$ 

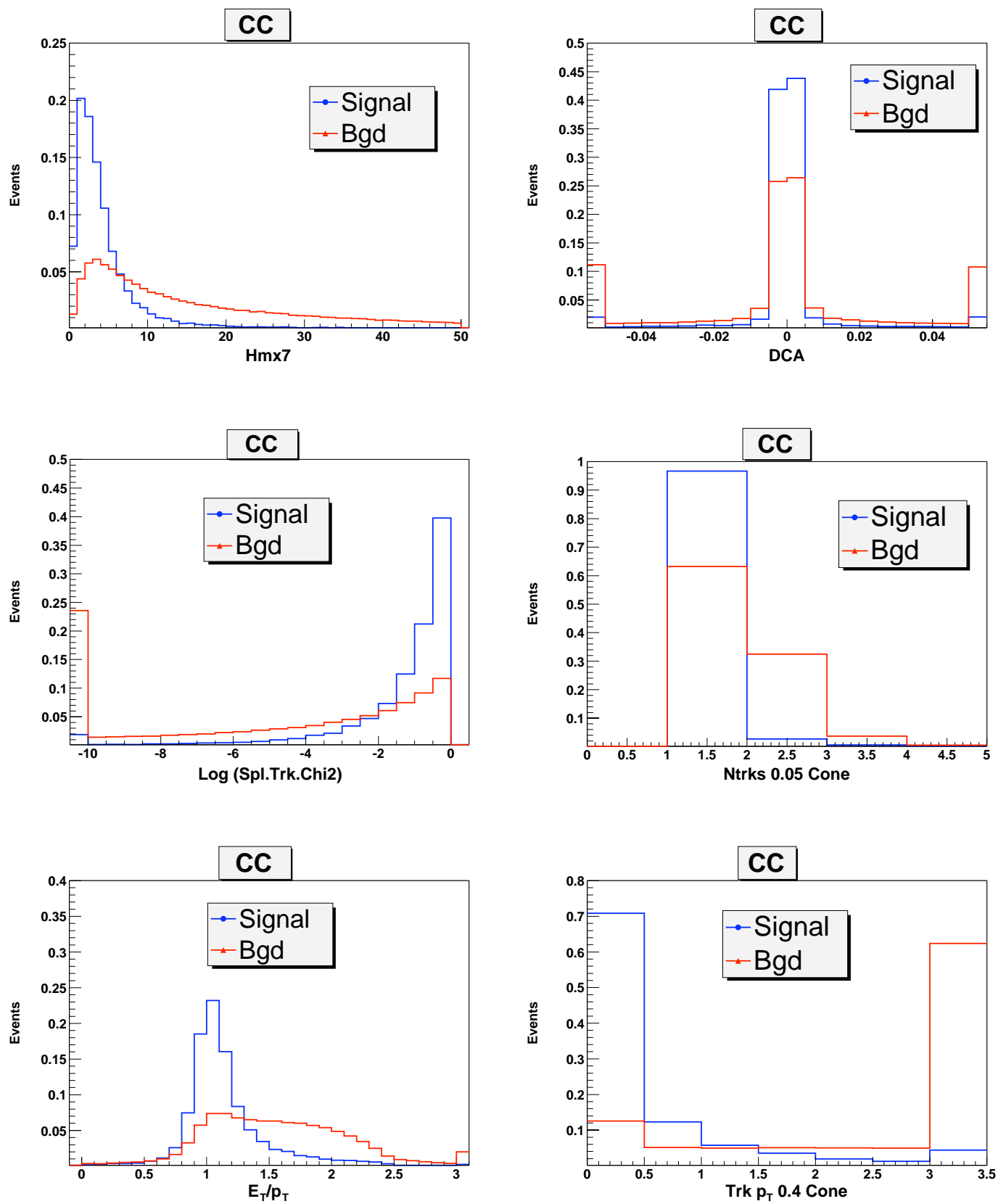

Figure 5.2: Electron discrimination variables distributions for real and fake electrons: H-matrix, $\log P\left(\chi_{\text {spatial }}^{2}\right)$, and $E_{\mathrm{T}} / p_{\mathrm{T}}$ down the first column; DCA, the number of tracks in a cone of size $R=0.05$, and the sum of the transverse momenta of all the tracks other than the associated track in a cone of size $R=0.4$ down the second column [69] 
A plot of the total efficiency for data and MC as a function of $\eta_{\text {det }}$ is given in Figure 5.4.

The loosest version that requires a track-match is called loose_trk, and is defined by:

- Fulfill Preselect_iso20 quality requirements

$-f_{\text {iso }} \leq 0.2$

$-f_{\mathrm{EM}} \geq 0.9$

$-p_{\mathrm{T}} \geq 3.0$

- Likelihood $\geq 0.2$. This implies:

- Spatial track-match $\chi^{2} \geq 0.0$

$-\mathrm{H}-\operatorname{Matrix}(7) \leq 50$

A plot of the total efficiency for data and MC as a function of $\eta_{\text {det }}$ is given in Figure 5.5. The efficiency in the EC suffers from the fact that there is no tracking coverage in the forward region. The dip around $\eta_{\text {det }} \approx 0$ is a result of incomplete modeling of tracking efficiencies in the $\mathrm{MC}[72]$.

\subsection{Muons}

Muons are reconstructed using either the muon detector and toroid only ("local") or the muon detector, toroid, plus the tracking detectors ("central track-matched"). To be considered a muon, the charged particle must have a minimum $p_{\mathrm{T}}>1.5 \mathrm{GeV}$. The muon quality is determined by how many hits the muon has associated with it in each layer of the muon system. 

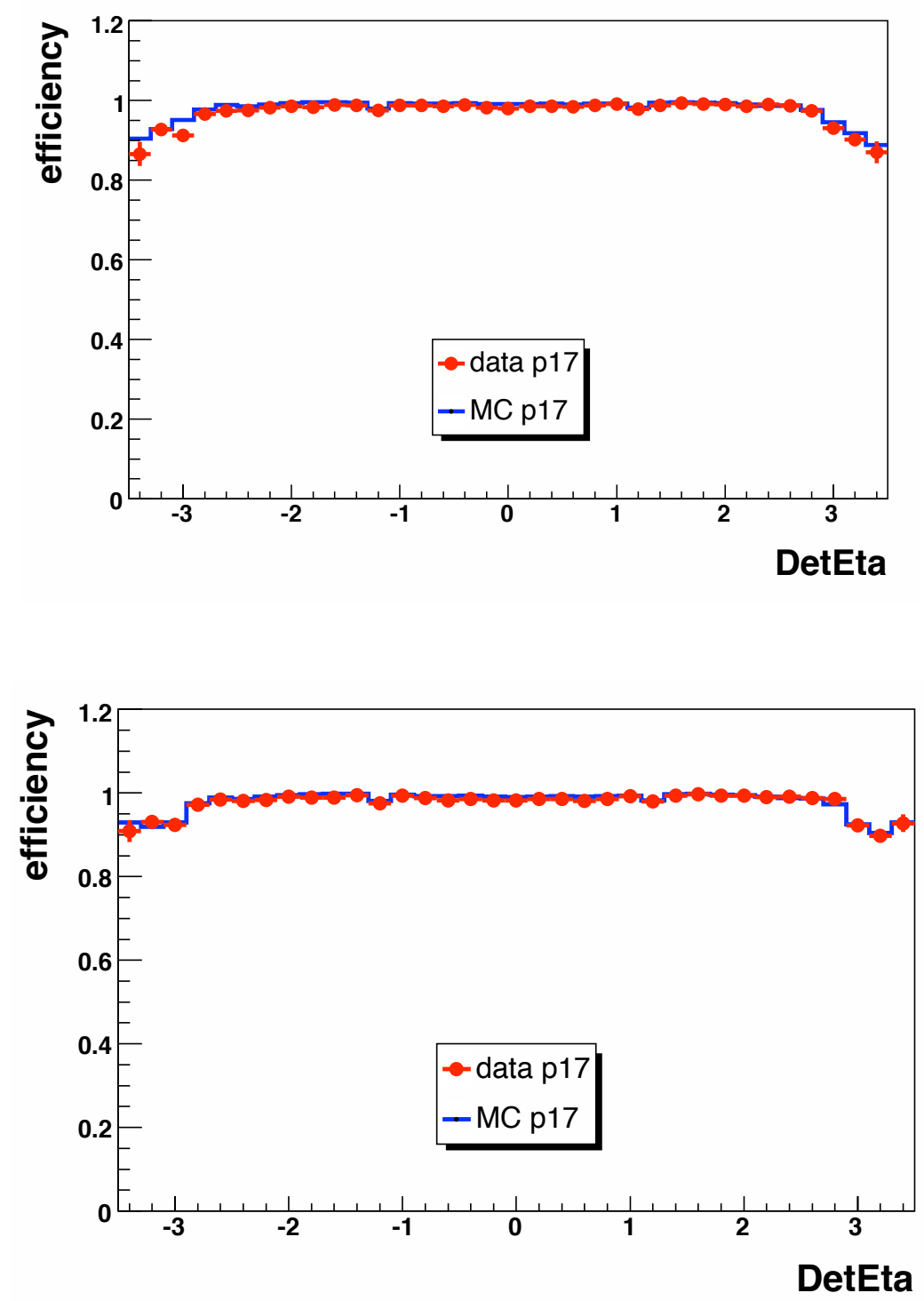

Figure 5.3: Efficiencies for data and MC electrons with quality loose_notrk as a function of $\eta_{\text {det }}$. A slight difference between the two plots is that the upper plot uses $\mathrm{H}-\operatorname{Matrix}(7) \leq 50$ for all $\eta_{\text {det }}$, while the bottom uses $\mathrm{H}-\operatorname{Matrix}(8) \leq 75$ for all $\eta_{\mathrm{det}}$. Thus, the upper plot is used for CC electrons, and the bottom for the EC [70]. 

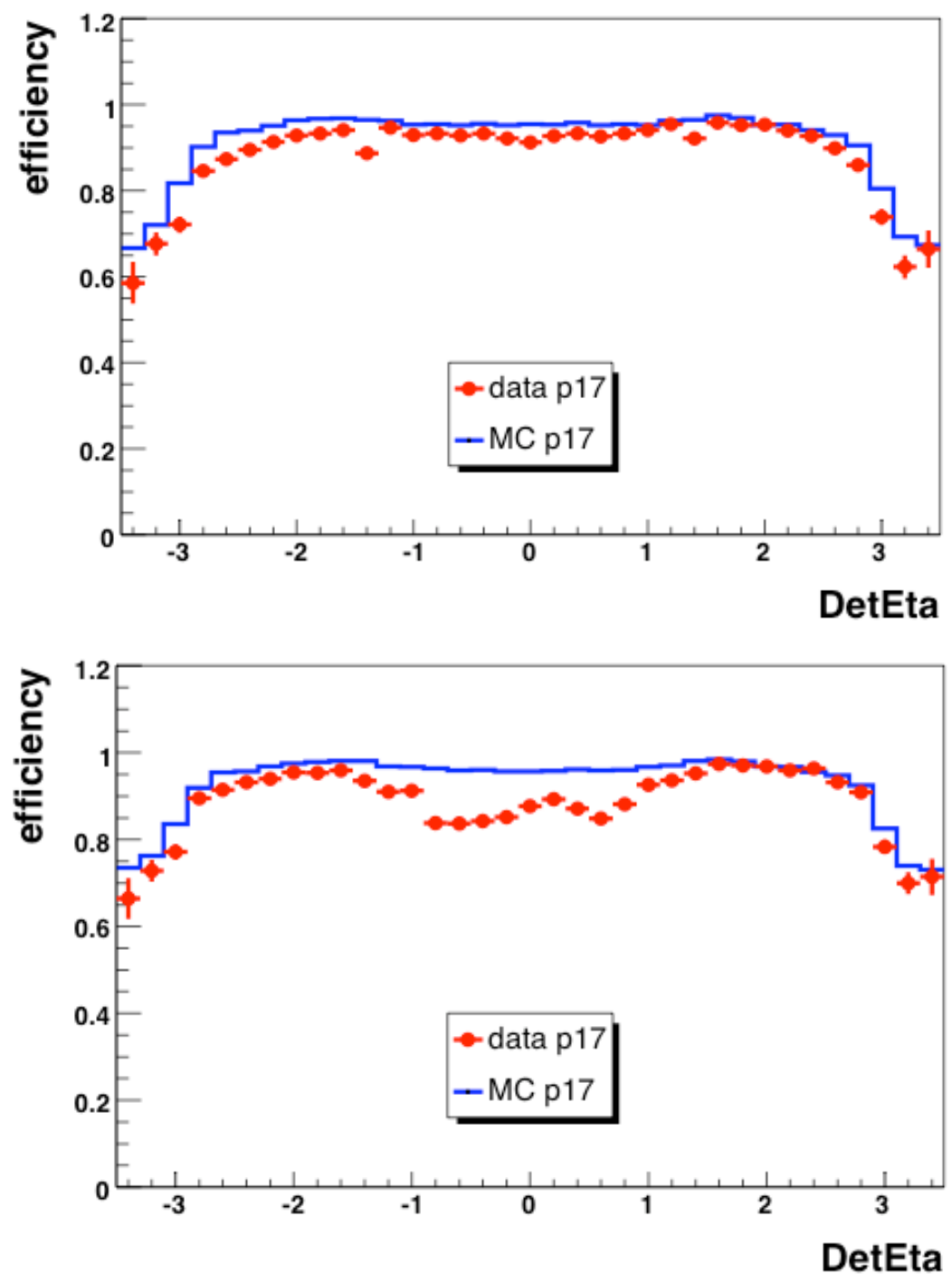

Figure 5.4: Efficiencies for data and MC electrons with quality tight_notrk as a function of $\eta_{\text {det }}$. A slight difference between the two plots is that the upper plot uses $\mathrm{H}-\operatorname{Matrix}(7) \leq 12$ for all $\eta_{\text {det }}$, while the bottom uses $\mathrm{H}-\operatorname{Matrix}(8) \leq 20$ for all $\eta_{\text {det }}$. Thus, the upper plot is used for CC electrons, and the bottom for the EC [70]. 


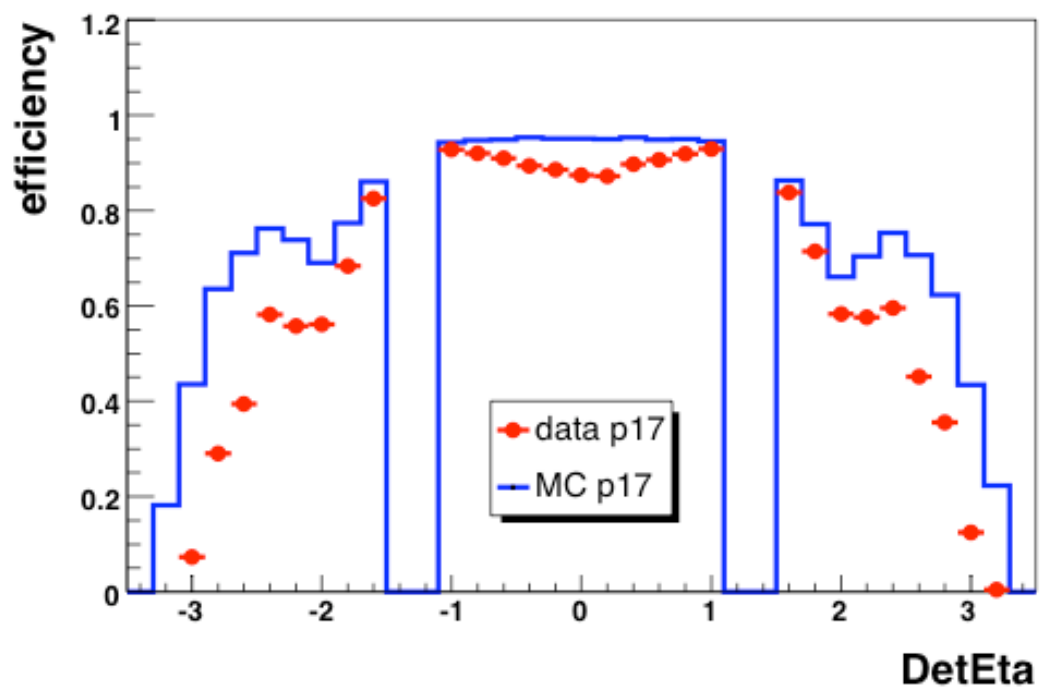

Figure 5.5: Efficiencies for data and MC electrons with quality loose_trk as a function of $\eta_{\text {det }}[70]$.

\subsection{Jets}

Jets are reconstructed from calorimeter information using the cone algorithm. Towers of size $\Delta \eta \times \Delta \times \phi=0.1 \times 0.1$ that have an energy above $1 \mathrm{GeV}$ or more are used as seeds in preclusters. Preclusters are formed by combining adjacent calorimeter towers within a radius of 0.3 to the seed towers. Jet clusters are defined by preclusters in a cone size $\Delta r=0.5$ or 0.7 around the jet centroid. Jets with $E_{\mathrm{T}}<8 \mathrm{GeV}$ are discarded. If two jets share the same tower, a split/merge fraction is calculated, which is the ratio of the shared energy of the jets to the energy of the least energetic jet. If the ratio is larger than $50 \%$, the jets are merged and a new centroid is calculated. Otherwise, the shared towers are split between the jets. 


\subsection{Missing Transverse Energy}

We are unable to measure the energy or the momentum of the fragments of the collision that go down the beam pipe. However, one can use the conservation of transverse momentum to indirectly detect weakly interacting, neutral particles (such as neutrinos) that would escape detection otherwise. The overall transverse momentum imbalance in the event is called Missing Transverse Energy $\left(\mathbb{E}_{T}\right)$. The $\mathbb{E}_{T}$ is calculated by first determining the $\mathrm{x}$ and $\mathrm{y}$ components of the visible energy $E^{\mathrm{vis}}$, in the calorimeter:

$$
E_{x, y}^{v i s}=\sum_{\text {cells }} E_{i}^{x, y}
$$

Then the $\mathrm{x}$ and $\mathrm{y}$ components of the missing transverse energy are $\mathbb{E}_{T x}=-E_{x}^{v i s}$ and $\mathscr{E}_{T y}=-E_{y}^{v i s}$. The total $\mathscr{E}_{T}$ is given by $\mathscr{E}_{T}=\sqrt{\left(\mathscr{E}_{T x}\right)^{2}+\left(\mathscr{E}_{T y}\right)^{2}}$. After calculating $\mathbb{E}_{T}$ from the calorimeter cell-level energies, the value is corrected to account for electron energy scale corrections. Energy corrections are applied to electrons satisfying $E_{\mathrm{T}}>$ $5 \mathrm{GeV}, f_{E M}>0.9$, and $f_{i s o}>0.1$, and the $\mathscr{E}_{T}$ is corrected accordingly. 


\section{Chapter 6}

\section{Analysis}

\subsection{Data Selection}

Reconstructed data are stored on tapes and contain information from all the physics objects that are used in the analyses. Most analyses do not use the full dataset but only a subset containing the relevant objects for each one. This initial selection of objects is done centrally to avoid duplication of work and is called "skimming". The resulting datasets are called "skims". This analysis uses the skim that contains at least two high $p_{\mathrm{T}}$ EM candidate objects with $p_{\mathrm{T}}>12 \mathrm{GeV}$ and $|I D|=10$ or $11^{1}$, and it is called "2EMhighpt". These selection criteria are very loose, and 36, 306, 915 events are contained in the "2EMhighpt" skim.

Starting with the 2EMhighpt skim, candidate events must have been declared good for tracking and calorimetry. Subsequently events are required to have at least two EM objects with $p_{\mathrm{T}}>25 \mathrm{GeV}$. For events with more than two EM objects, the

${ }^{1}|I D|=10$ is awarded to electron candidates that are found by the cluster algorithm but there is no track match, whereas $|I D|=11$ is awarded to electron candidates that are found by the cluster algorithm and have track match confirmation 
two with the highest $p_{\mathrm{T}}$ are selected. Depending on the location of the EM objects in the calorimeter, three subsets are created:

- CCCC, where both EM objects are in the central calorimeter,

- ECCC, where one EM object is in the central calorimeter, and the other is in the end-cap,

- ECEC, where both EM objects are in the end-caps.

For CCCC, one EM object is required to have an associated track and satisfy the loose_trk electron quality, while the second EM object just needs to satisfy the loose_notrk electron quality definition. For ECCC, the EM object in the central calorimeter is required to have an associated track and satisfy the loose_trk electron quality, while the EM object in the end-cap is required to satisfy the tighter tight_notrk electron quality definition, in order to reduce the QCD dijet background. Detailed descriptions of the electron quality definitions used can be found in Section 5.4. Due to the limited tracking in the forward region the ECEC topology is dominated by QCD dijet processes, which are a major background for this analysis. For QCD events with invariant mass $>500 \mathrm{GeV}$ about $85 \%$ of the events are in the ECEC. Additionally, for the high masses relevant to this analysis, only a very small fraction of signal events ( $\sim 6 \%$ for a $\mathrm{Z}^{\prime}$ of $\left.900 \mathrm{GeV}\right)$ end up in the ECEC topology. Thus, the ECEC topology will not be used in this analysis. Regardless of the topology, electron quality definitions that require an associated track will be referred to as "tight" cuts, whereas the quality definitions that do not require an associated track will be identified as "loose" cuts.

For the CCCC topology the full 2EMhighpt skim is used, resulting in an integrated luminosity of $(1106 \pm 67) \mathrm{pb}^{-1}$. The ECCC topology was impacted by a cabling 
swap issue that affected the endcap calorimeters from December 2005 till the end of Run IIa in February 2006. The integrated luminosity ignoring that period of data taking is $(1007 \pm 61) \mathrm{pb}^{-1}$.

\subsection{Backgrounds}

The major backgrounds for the $\mathrm{Z}^{\prime} \rightarrow$ ee process are physics processes that have true dielectron final states, such as Z/Drell-Yan (DY) production, and from QCD events where jets are misidentified as isolated electrons.

\subsubsection{Physics Backgrounds}

\section{Z/Drell-Yan Background}

The dominant physics background to the $\mathrm{Z}^{\prime}$ sample is the $Z /$ Drell-Yan production and decay:

$$
q \bar{q} \rightarrow Z / \gamma^{\star} \rightarrow e e
$$

This process is modeled using Monte Carlo (MC) simulation. The DY MC events have been generated using PYTHIA [73] with CTEQ6L parton distribution functions, and then processed through the standard D0 detector simulation based on GEANT3 [74]. MC events are required to satisfy the same selection criteria as data. To ensure enough statistics in the high mass region, samples have been generated in four different mass ranges and then joined together based on their next to leading order (NLO) cross sections. Table 6.1 lists the leading order (LO) cross sections and the number of events for each sample. The cross section produced by PYTHIA is only to leading 


\begin{tabular}{|ccc|}
\hline Mass Window & LO Cross Section $(\mathrm{pb})$ & Number of Events \\
\hline \hline $60-130$ & 178 & 109500 \\
$130-250$ & 1.3 & 27500 \\
$250-500$ & 0.11 & 27000 \\
$>500$ & 0.0045 & 25500 \\
\hline
\end{tabular}

Table 6.1: List of DY MC samples and LO cross sections.

order. A k-factor needs to be applied to account for NLO calculations. A mass independent k-factor of 1.3 [75] has been chosen. Variations of the k-factor due to mass dependency are considered as a systematic error.

PYTHIA, being a leading order MC generator, leads to the spectrum of the transverse momentum $p_{T}$ of the $Z$ boson not being properly described when jets are present, due to higher order contributions. Thus a weight needs to be applied to the $p_{T}$ spectrum to correct the description. A specific tool has been developed by D0 that calculates these weights by comparing events generated by ALPGEN [76] to those generated by PYTHIA. When these weights are applied a slight over-correction is noticed (see Figure 6.1), thus it was decided to take the average between the corrected distribution and the uncorrected one, and consider the difference as a systematic.

The normalized invariant mass distribution from $\mathrm{MC}$ are given in Figure 6.2 for CCCC and ECCC samples. The spikes that can be seen in both cases around $250 \mathrm{GeV}$ are due to statistical fluctuations near the boundaries between the various MC datasets, and are not relevant for the analysis.

\section{Other Physics Backgrounds}

Apart from the $Z$ /Drell-Yan process, other physics background processes that produce dielectron final states include $[22,77]$ : 

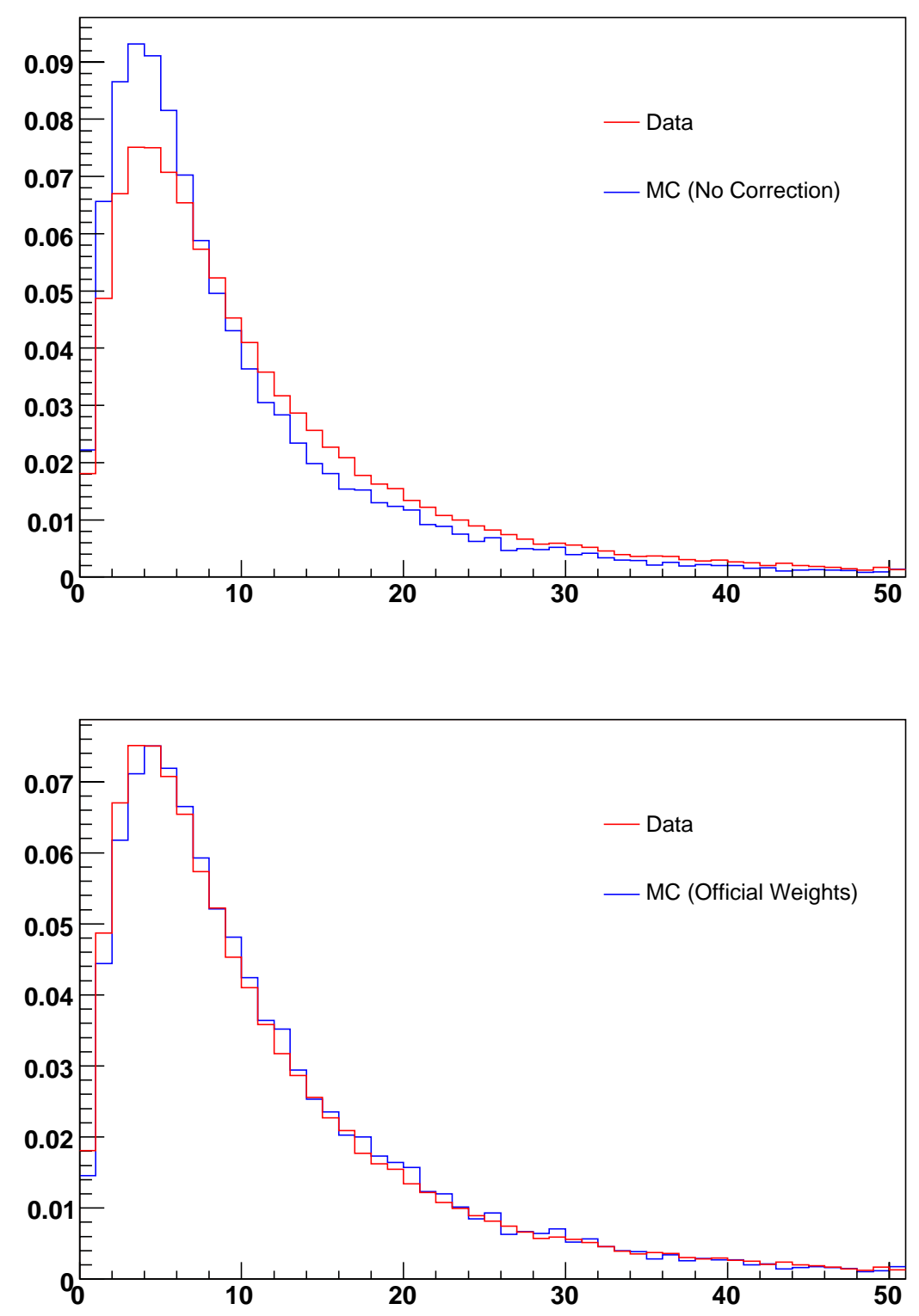

Figure 6.1: Comparisons of the $p_{T}$ spectrum of the $Z$ boson between data and MC. The upper plot corresponds to uncorrected MC, and the bottom after having applied the official D0 weighting. 

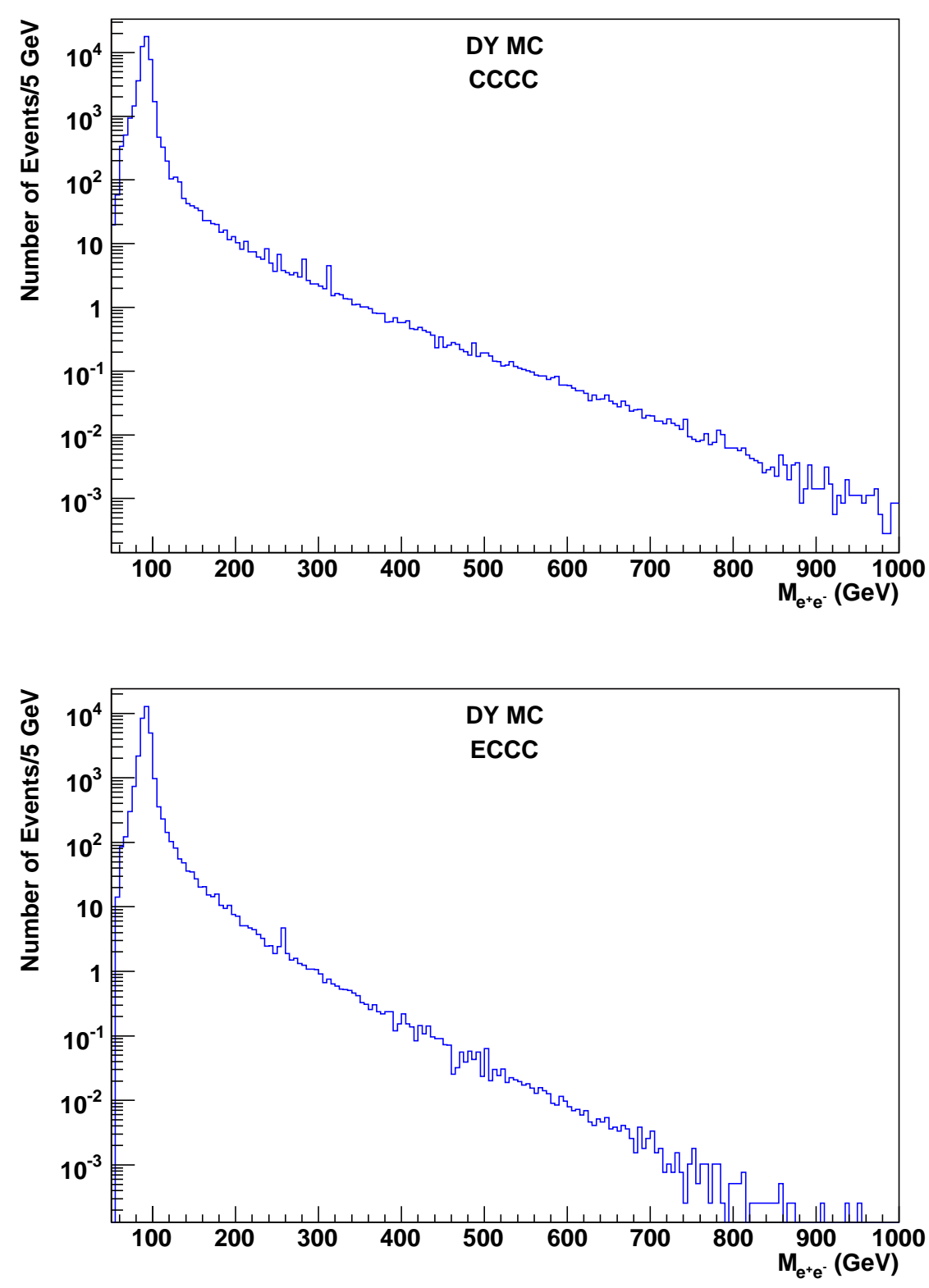

Figure 6.2: Dielectron invariant mass distribution of the DY background from MC. The upper plot corresponds to the CCCC topology, and the bottom to the ECCC. The number of events are normalized accordingly to the integrated luminosity of the data. 
- Boson pair production; $W W \rightarrow e e, W Z \rightarrow e e, Z Z \rightarrow e e$, with $\sigma_{N L O}=(11.5,3.58,1.42) \mathrm{pb}$ respectively.

- $t \bar{t}$ production where the $W$ s decay into electrons $(t \bar{t} \rightarrow e e)$, with $\sigma_{N N L O}=0.012 \mathrm{pb}$

- Processes that result in a final state of an electron and a photon, where the electron passes the "tight" cuts and the photon passes "loose" cuts, like $W \gamma \rightarrow$ $e \gamma$ and $Z \gamma \rightarrow e \gamma$.

The $W \gamma \rightarrow e \gamma, Z \gamma \rightarrow e \gamma$ backgrounds produce numbers of events which are about two orders of magnitude smaller than from the DY background. The other backgrounds are smaller still. As a result, the contributions from physics backgrounds other than Drell-Yan are neglected.

\subsubsection{Instrumental Background - Misidentified Electrons}

The main source of instrumental background arises from QCD multijet events in which jets have been misidentified as electrons. This can happen when a jet is formed with most of its energy being carried by an isolated $\pi^{0}$ or $\eta$ which then decays into a pair of spatially close photons. Such a photon pair might be indistinguishable in the calorimeter and would therefore be reconstructed as a single photon, and would pass the "loose" electron quality identification requirements. Additionally, tracks can be associated with the EM object formed by the photon pair, either by charged hadrons from the jet forming tracks that overlap with the EM object, or by one of the photons converting to $e^{+} e^{-}$that will form tracks that can be associated with the EM object. Such events can possibly pass the "tight" electron quality identification requirements. 
The instrumental background is estimated from D0 data. Starting from the same 2EMhighpt skim, events that contain at least two EM objects with $p_{\mathrm{T}}>25 \mathrm{GeV}$ are selected. These EM objects are additionally required to satisfy the isolation and EM fraction cuts applied for electrons. However, they must fail the H-Matrix cut. Thus, for CC fake EM objects are selected that satisfy:

- $f_{\text {iso }} \leq 0.2$

- $f_{E M} \geq 0.9$

- $\mathrm{H}-\operatorname{Matrix}(7)>50,(\mathrm{H}-\operatorname{Matrix}(7) \leq 50$ is the selection cut for electrons $)$

while for EC, fake EM objects need to satisfy:

- $f_{\text {iso }} \leq 0.2$

- $f_{E M} \geq 0.9$

- $\mathrm{H}-\operatorname{Matrix}(8)>20,(\mathrm{H}-\operatorname{Matrix}(7) \leq 20$ is the selection cut for electrons $)$.

For an event to be selected both of the objects need to satisfy the "misidentification" requirements. If there are more than two objects in an event that satisfy the above requirements, the two with the highest $p_{\mathrm{T}}$ are selected. These events are used to obtain an estimation of the shape of the invariant mass spectrum for events with misidentified electrons. The shapes of the invariant mass spectrum for CCCC and ECCC are given in Figure 6.3.

\subsection{Data Treatment}

In Figure 6.4 the measured dielectron mass distribution, for the full studied region, is shown for the CCCC and ECCC topologies. The area of interest for the $\mathrm{Z}^{\prime}$ search is 

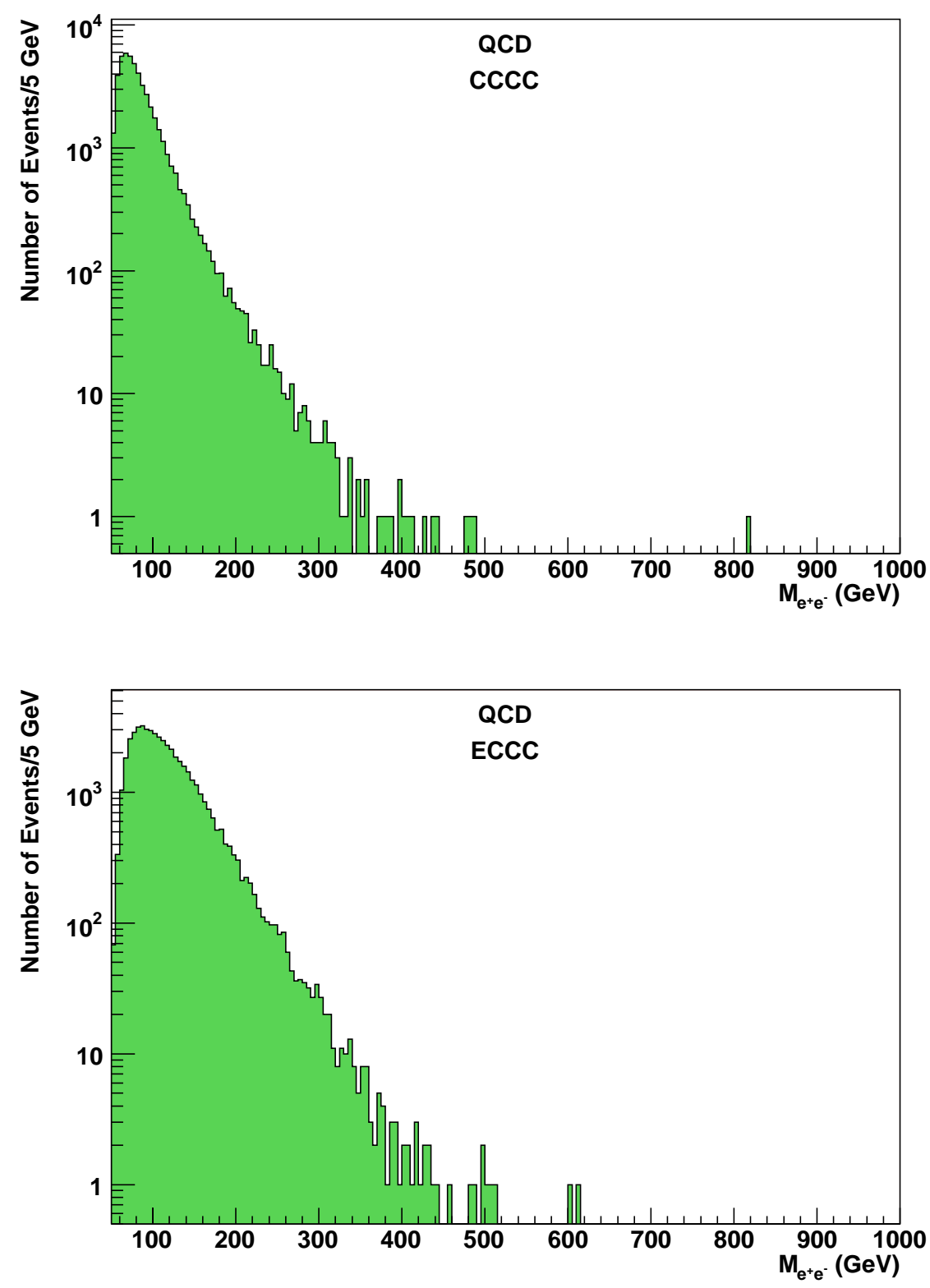

Figure 6.3: Invariant mass spectrum of events with misidentified electrons, as described in the text. The upper plot corresponds to the CCCC topology, and the bottom to the ECCC. 

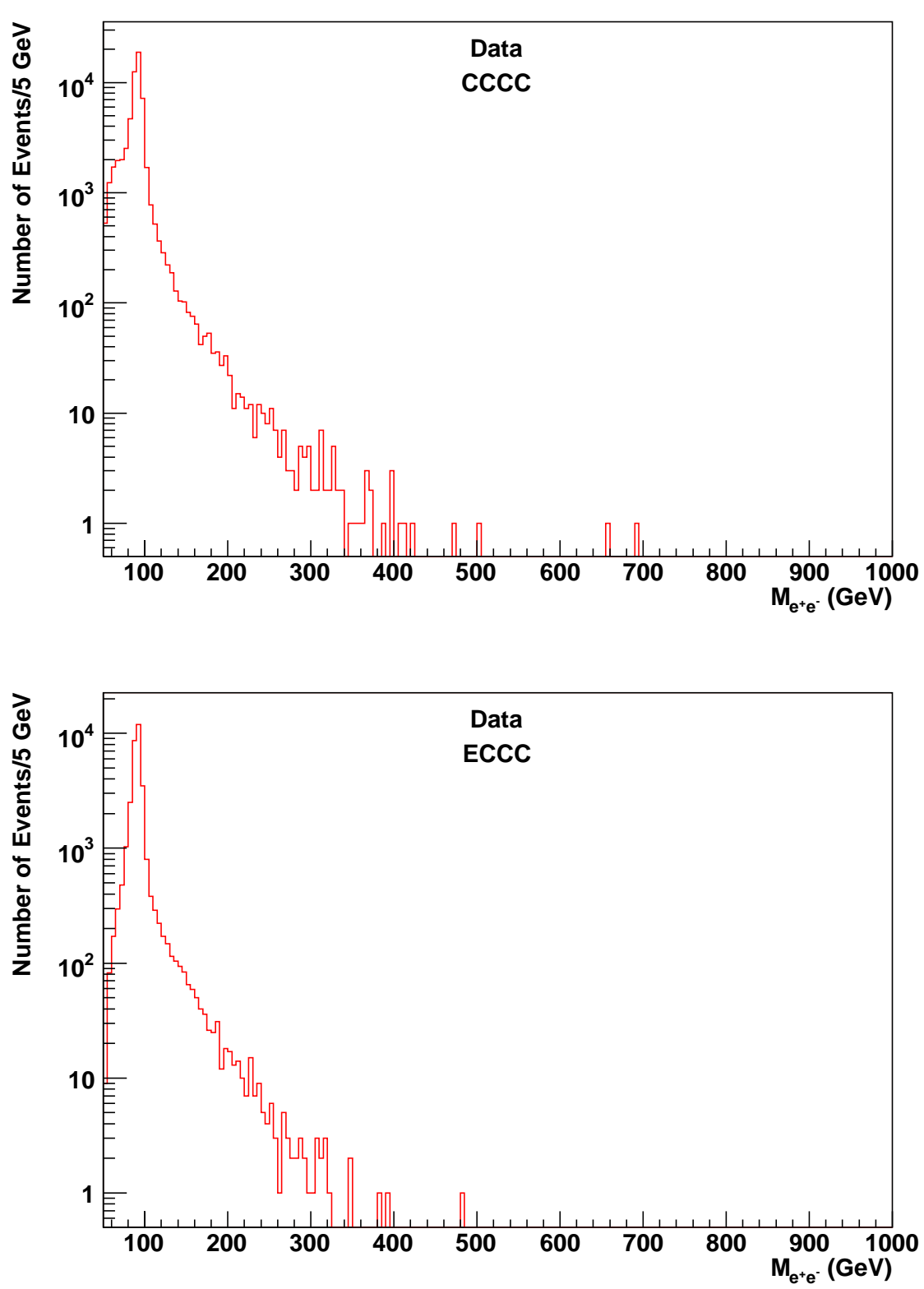

Figure 6.4: Dielectron invariant mass distribution of the data. The upper plot corresponds to the CCCC topology, and the bottom to ECCC. 
$M_{e e}>300 \mathrm{GeV}$. To estimate the contribution of physics and instrumental background in that range, it is essential to have a good description of the $Z$ peak region. A Breit-Wigner distribution convoluted with a Gaussian is chosen for the description of the $Z$ peak in the data, whereas the background is modeled by a third-order polynomial. The width of the Breit-Wigner is set to the PDG value of the width for the $Z$ resonance, $\Gamma_{Z}=2.4952 \mathrm{GeV}[7]$. Figure 6.5 shows the invariant mass distributions with the fit for the CCCC and ECCC topologies. The fit range in $\mathrm{GeV}$ is $80 \leq M_{e e} \leq 110$ for CCCC and $80 \leq M_{e e} \leq 115$ for ECCC. For the CCCC topology the fit peaks at $91.355 \pm 0.029 \mathrm{GeV}$ and has a sigma of $3.112 \pm 0.037 \mathrm{GeV}$. In the ECCC topology, fitting in the same range, the peak is at $90.988 \pm 0.029 \mathrm{GeV}$ with a sigma of $2.927 \pm 0.036 \mathrm{GeV}$. The sigma of the fits are consistent with the expected detector resolution.

In the CCCC topology the fitted mass is slightly shifted from the PDG value of $M_{Z}=91.1876 \mathrm{GeV}$. For ECCC there is a smaller discrepancy. The main source is due to the imperfect modeling of the non-fiducial areas of the central calorimeter ( $\phi$ cracks ), for which the scale and smearing factors in the D0 reconstruction program do not describe those areas of the calorimeter accurately. Therefore electrons in the $\mathrm{CC}$ are distinguished according to whether they are "in fiducial" and "non-fiducial", and the CCCC topology splits to three sub-samples:

- both electrons are in fiducial,

- one electron is in fiducial,

- none of the electrons is in fiducial,

where an electron is defined to be in fiducial if the center of the EM shower, as defined from the cone algorithm, lies in the fiducial area of the central calorimeter [78]. The 

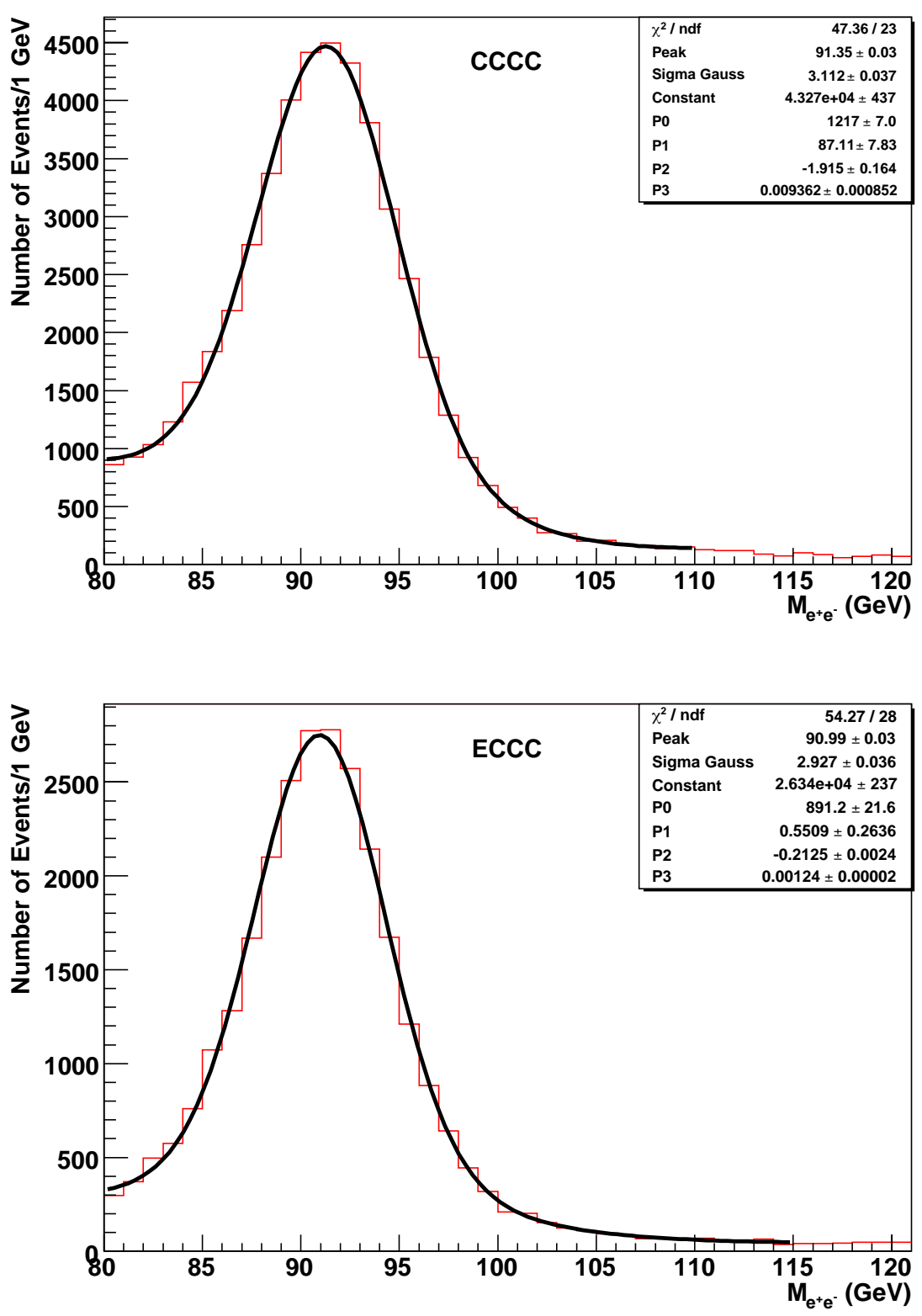

Figure 6.5: Invariant mass distribution around $Z$ peak fitted with a BreitWigner convoluted with a Gaussian. The upper plot corresponds to the CCCC topology, and the bottom to the ECCC. 
ECCC sample is split in a similar fashion to two sub-samples, the in fiducial (ECinCC) and the non-fiducial (ECoutCC) depending on the location of the EM cluster in the central calorimeter ${ }^{2}$.

Applying the same fitting function around the $Z$ peak to the five sub-samples, the peak and the width for each distribution are determined. The results are summarized in Table 6.2 (the distributions and the fits are shown in Figures 6.6 for the CCCC, and 6.7 for the ECCC). From the results it becomes apparent that a correction is needed to be applied to the non-fiducial electrons. Additionally, the EC electron needs a slight correction.

\begin{tabular}{|c|c|c|c|c|}
\hline Topology & Sub-sample & Fit Range $(\mathrm{GeV})$ & Peak $(\mathrm{GeV})$ & Sigma $(\mathrm{GeV})$ \\
\hline \hline \multirow{3}{*}{ CCCC } & both in fiducial & $80-115$ & $91.514 \pm 0.025$ & $2.97 \pm 0.031$ \\
& one in fiducial & $79-105$ & $90.24 \pm 0.09$ & $4.31 \pm 0.11$ \\
& none in fiducial & $70-104$ & $87.9 \pm 0.3$ & $10.4 \pm 0.7$ \\
\hline \multirow{2}{*}{ ECCC } & in fiducial & $75-115$ & $91.108 \pm 0.03$ & $2.78 \pm 0.03$ \\
& non-fiducial & $75-105$ & $89.85 \pm 0.14$ & $3.78 \pm 0.16$ \\
\hline
\end{tabular}

Table 6.2: $Z$ peak fit results for fiducial sub-samples.

\subsubsection{Correction for Non-fiducial Electrons}

The correction for the non-fiducial electrons is performed based on the "one in fiducial" sub-sample. For that sample, it is assumed that the energy $E_{f i d}$ of the fiducial electron and the angle $\theta$ between the directions of the two electrons are measured precisely. Neglecting the electron masses, in the high energy limit, the invariant mass

\footnotetext{
${ }^{2}$ The endcap calorimeters are, by construction, not affected by $\phi$-cracks.
} 

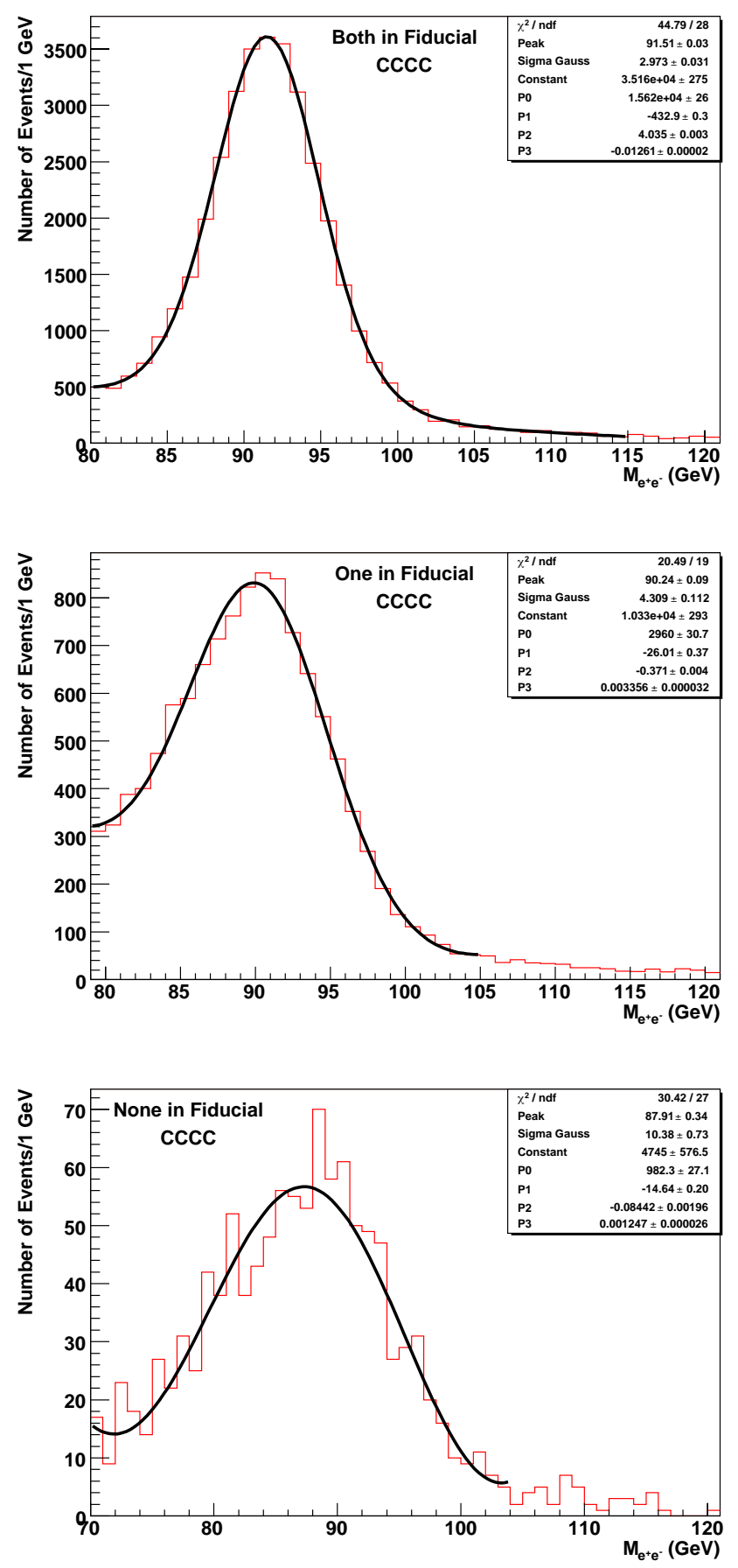

Figure 6.6: Invariant mass distribution around $Z$ peak fitted with a BreitWigner convoluted with a Gaussian corresponding to the CCCC sub-samples. The upper plot corresponds to the both in fiducial sub-sample, the middle to the one in fiducial sub-sample, and the bottom to the none in fiducial sub-sample. 

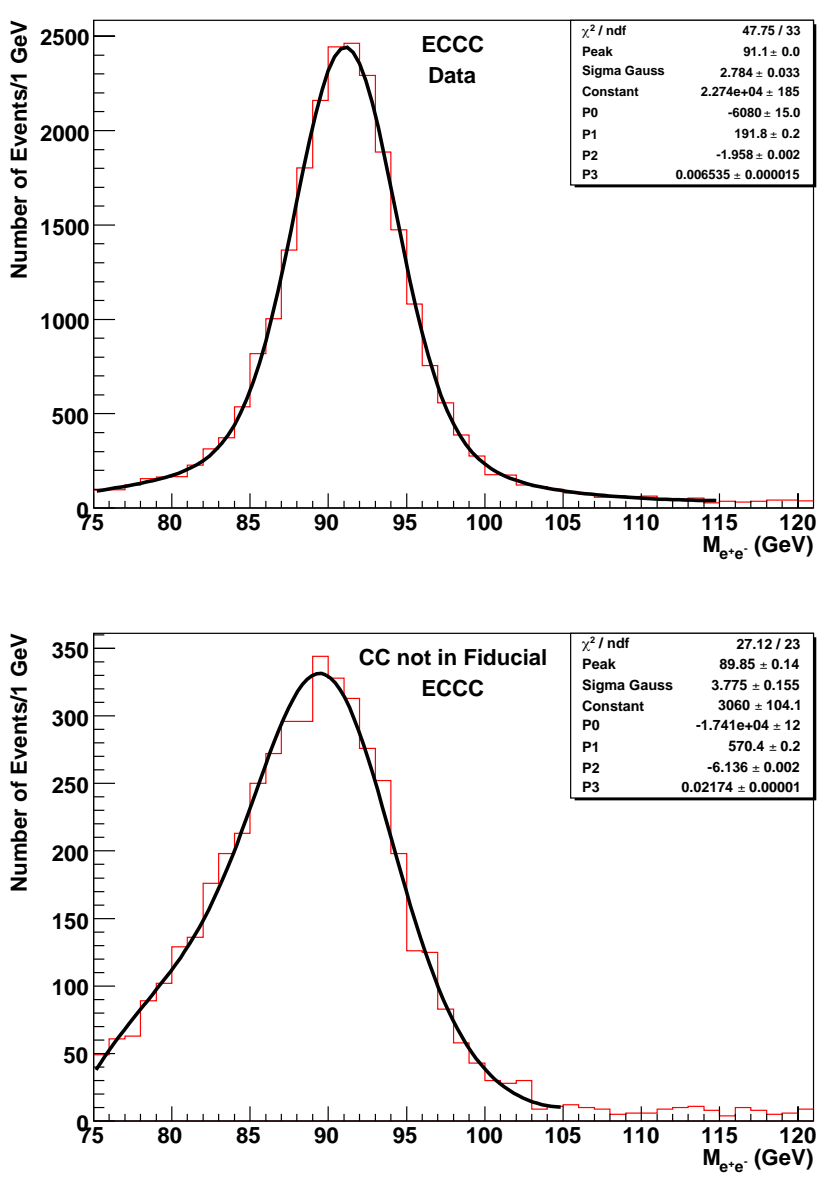

Figure 6.7: Invariant mass distribution around $Z$ peak fitted with a BreitWigner convoluted with a Gaussian corresponding to the ECCC sub-samples. The upper plot corresponds to the in fiducial sub-sample, and the bottom to the one in fiducial sub-sample. 
$M_{i n v}$ for an electron pair is given by:

$$
M_{i n v}=\sqrt{2 E_{f i d} E_{n o n}(1-\cos \theta)}
$$

where $E_{n o n}$ is the energy of the non-fiducial electron. For events with invariant mass around the $Z$ peak $\left(75 \mathrm{GeV} \leq M_{i n v} \leq 105 \mathrm{GeV}\right)$, a correction $\Delta E$ needs to be added to $E_{n o n}$ in order to get the central value of $Z$ mass $M_{Z}$ :

$$
M_{Z}=\sqrt{2 E_{f i d}\left(E_{n o n}+\Delta E\right)(1-\cos \theta)}
$$

Squaring and then subtracting equations 6.3 and 6.2 , the correction $\Delta E$ is given by:

$$
\Delta E=\frac{M_{Z}^{2}-M_{i n v}^{2}}{2 E_{f i d}(1-\cos \theta)}
$$

$\Delta E$ determined in this manner gives the appropriate correction for events under the $Z$ peak but not necessarily for events in the high mass region of interest. In order to estimate the degree of correction needed, the behavior of the ratio $\alpha=E_{\text {cor }} / E_{\text {uncor }}$ as a function of $E_{\text {uncor }}$ is studied $\left(E_{\text {uncor }}=E_{\text {non }}\right)$, for events under the $Z$ peak (Figure 6.8). Then those data are fitted with two empirically determined functional forms:

$$
\begin{gathered}
\alpha=1+e^{C_{1} \times E_{\text {uncor }}} \\
\text { and } \alpha=A\left(1+B \cdot e^{C \times E_{\text {uncor }}}\right)
\end{gathered}
$$

The more energetic the electron, the wider the shower is expected to be, and it will therefore extend to the fiducial part of the central calorimeter. The measurement of high energy electrons is therefore expected to require a smaller correction, an expectation supported by Figure 6.8. In Equation 6.5 it is considered that no correction is 


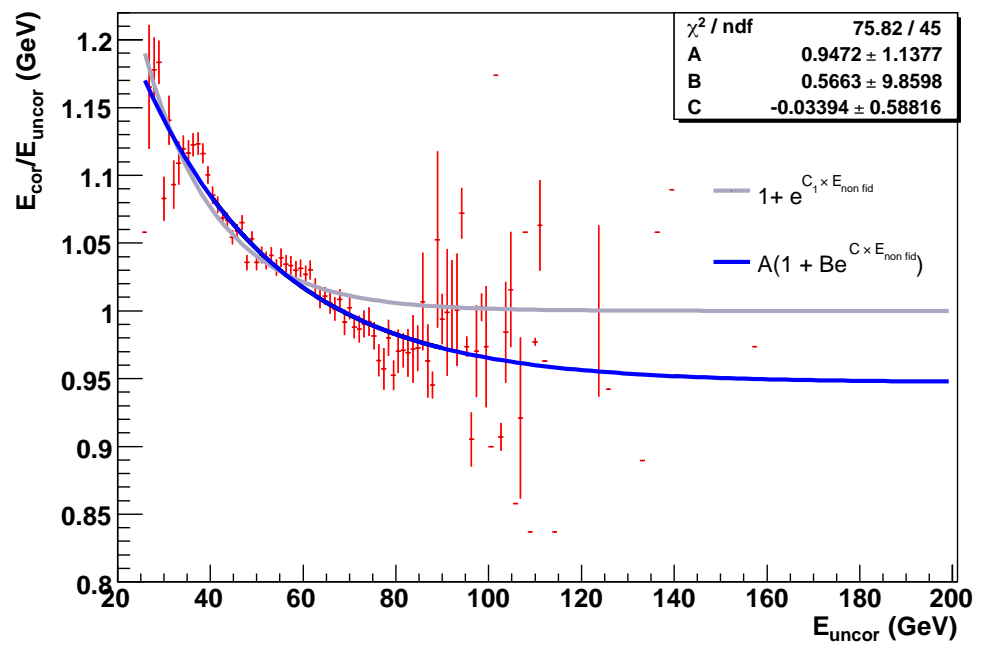

Figure 6.8: Correction $\alpha=E_{\text {cor }} / E_{\text {uncor }}$ as a function of uncorrected energy $E_{\text {uncor }}$ for non-fiducial electrons, and fit results. The fits have been performed in the region $35 \mathrm{GeV} \leq E_{\text {uncor }} \leq 85 \mathrm{GeV}$.

needed for high energy electrons, while in Equation 6.6 the degree of the correction is determined by the fit and is calculated to be 0.95 . The fit is applied to the region $35 \mathrm{GeV} \leq E_{\text {uncor }} \leq 85 \mathrm{GeV}$ and then extrapolated to higher energies. The difference between the two functional forms will be used for the calculation of the systematic errors.

With the correction factor $\alpha$ being determined, the correct invariant mass is calculated:

$$
\begin{aligned}
M_{i n v}^{2}= & \left(E_{f i d}+\alpha \cdot E_{n o n}\right)^{2} \\
& -\alpha \cdot\left(P_{X_{f i d}}+P_{X_{n o n}}\right)^{2} \\
& -\alpha \cdot\left(P_{Y_{f i d}}+P_{Y_{\text {non }}}\right)^{2} \\
& -\alpha \cdot\left(P_{Z_{f i d}}+P_{Z_{n o n}}\right)^{2}
\end{aligned}
$$


From Figure 6.6 one sees that the sub-sample with no electrons in fiducial has a relatively poor resolution, and also makes only a small contribution to the signal ${ }^{3}$. Therefore this sub-sample is discarded in the $\mathrm{Z}^{\prime}$ analysis, and for the CCCC topology only the sub-samples with both electrons in fiducial, and one electron in fiducial are used.

\subsubsection{Correction for End-Cap Electrons}

The energy of the electron in the endcap calorimeter $\left(E_{E C}\right)$ can be corrected using a similar method as in Section 6.3.1. Starting from the "in fiducial" ECCC sub-sample, is assumed that the energy of the in fiducial electron in the $\mathrm{CC}\left(E_{C C}\right)$ and the angle $\theta$ between the directions of the two electrons are measured precisely. Following the same line of arguments as in Section 6.3.1, $E_{E C}$ needs to be corrected by a quantity $\Delta E$ where:

$$
\Delta E=\frac{M_{Z}^{2}-M_{i n v}^{2}}{2 E_{C C}(1-\cos \theta)}
$$

The corrected energy for the EC electron then becomes: $E_{c o r}=E_{E C}+\Delta E$. The ratio $\alpha^{\prime}=E_{\text {cor }} / E_{\text {uncor }}\left(E_{\text {uncor }}=E_{E C}\right)$ as a function of detector $\left|\eta_{E C}\right|$ is studied for events under the $Z$ peak. A third degree polynomial is used as an empirical fitting function.

\subsection{Background Normalization}

We normalize the background around the $Z$ peak where there is a lot of statistics in the data spectrum, and the physics processes are well understood. Then the

\footnotetext{
${ }^{3}$ This sample was used only to cross-check the invariant mass distribution, after applying the non-fiducial correction to both electrons.
} 


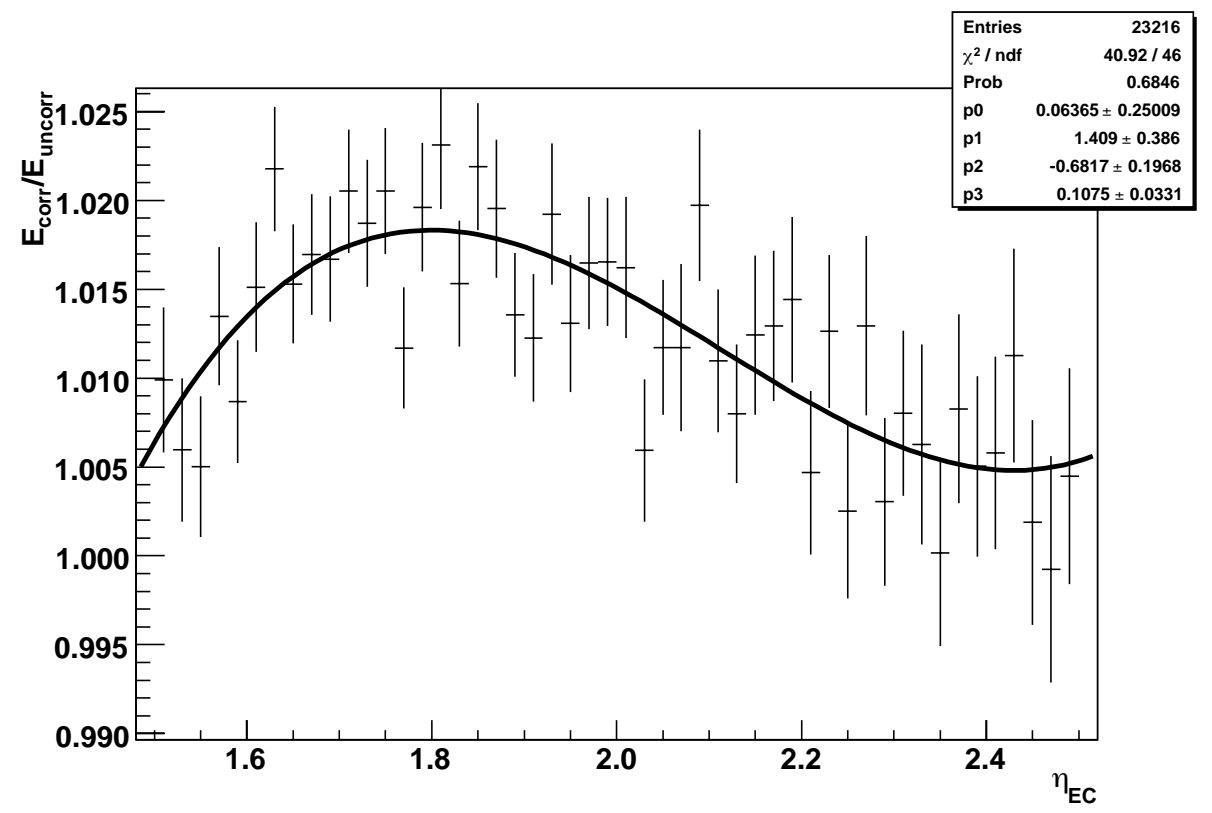

Figure 6.9: Correction $\alpha^{\prime}=E_{\text {cor }} / E_{\text {uncor }}$ as a function of detector $\left|\eta_{E C}\right|$.

normalization can be extrapolated to the high mass region. Around the $Z$ peak region, the invariant mass spectrum of the data $N_{\text {data }}$ is fitted with a superposition of the total physics background $N_{\text {phys }}$ and the instrumental background $N_{Q C D}$ :

$$
N_{\text {data }}(m)=N_{\text {bkgd }}(m)=f_{Q C D} \cdot N_{Q C D}(m)+f_{\text {phys }} \cdot N_{\text {phys }}(m)
$$

where $f_{\text {phys }}$ and $f_{Q C D}$ are normalization factors of the physics and instrumental background respectively. Integrated over the fit window, the total number of events in the data should equal the total number of events in the sum. Hence, there is only one degree of freedom in Equation 6.9, and the two normalization factors are connected by the relation $f_{\text {phys }}=1-f_{Q C D}$.

A binned $\chi^{2}$ minimization fit is performed, based on the MINUIT package that is 
included in the CERN ROOT library [79]. The parameter $f_{Q C D}$ is varied to minimize the $\chi^{2}$ between $N_{b k g d}$ and $N_{\text {data }}$, where $\chi^{2}$ is defined as:

$$
\chi^{2}=\sum_{i} \frac{\left[n_{i}-N \cdot\left(f_{Q C D} \cdot \alpha_{i}+\left(1-f_{Q C D}\right) \cdot b_{i}\right)\right]^{2}}{\delta^{2}}
$$

where

$$
\delta^{2}=n_{i}+\left(\frac{N f_{Q C D}}{N_{Q C D}}\right)^{2} n_{Q C D_{i}}+\left(\frac{N\left(1-f_{Q C D}\right.}{N_{\text {phys }}}\right)^{2} n_{\text {phys }_{i}}
$$

and

- $\alpha_{i}=\frac{n_{Q C D_{i}}}{N_{Q C D}}$ is the contents of bin $i$ of the normalized instrumental background spectrum,

- $b_{i}=\frac{n_{p h y s_{i}}}{N_{\text {phys }}}$ is the contents of bin $i$ of the normalized physics background spectrum.

- $n_{i}$ is the number of data events in bin $i$,

- $N=\sum_{i} n_{i}$, is the total number of data events within the fit range,

- $n_{Q C D_{i}}$ is the number of instrumental background events in bin $i$,

- $N_{Q C D}=\sum_{i} n_{Q C D_{i}}$ is the total number of instrumental background events within the fit range,

- $n_{\text {phys }_{i}}$ is the number of physics background events in bin $i$,

- $N_{\text {phys }}=\sum_{i} n_{\text {phys }_{i}}$ is the total number of physics background events within the fit range, 
The fit is performed in the $[60,140] \mathrm{GeV}$ region for the CCCC topology and in the $[70,150] \mathrm{GeV}$ region for the ECCC topology ${ }^{4}$. An additional fit is performed in the $[65,115] \mathrm{GeV}$ region for the CCCC $([75,125] \mathrm{GeV}$ for the ECCC). The two fits are extrapolated to high masses and the difference in extrapolation is included in the systematic error.

\subsubsection{CCCC}

The two sub-samples that are used (both electrons in fiducial and one electron in fiducial) are fitted independently, and then they are combined. Once the contributions of the instrumental and physics backgrounds are calculated for the two sub-samples, they are added bin-by-bin to construct the invariant mass spectrum for the backgrounds, and then compare it to the data. Figure 6.10 shows the comparison of the data and the expected background spectrums in the area around the $Z$ peak. The two distributions are in good agreement.

For the "both in fiducial" sub-sample, the result of the fit is $f_{q c d}=0.165 \pm 0.004$ (including the fit error). For the "one in fiducial" topology, $f_{q c d}=0.201 \pm 0.008$. Plots of each separate sub-sample can be found in Appendix A.

\subsubsection{ECCC}

For the ECCC topology, the "in fiducial" sub-sample is used, where the electron in the central calorimeter is in the fiducial area of the calorimeter. The result of the fit is $f_{q c d}=0.073 \pm 0.004$. The normalization factor for instrumental background $f_{Q C D}$

\footnotetext{
${ }^{4} \mathrm{~A}$ slightly different region is used for the ECCC topology due to the higher background in the low mass region.
} 

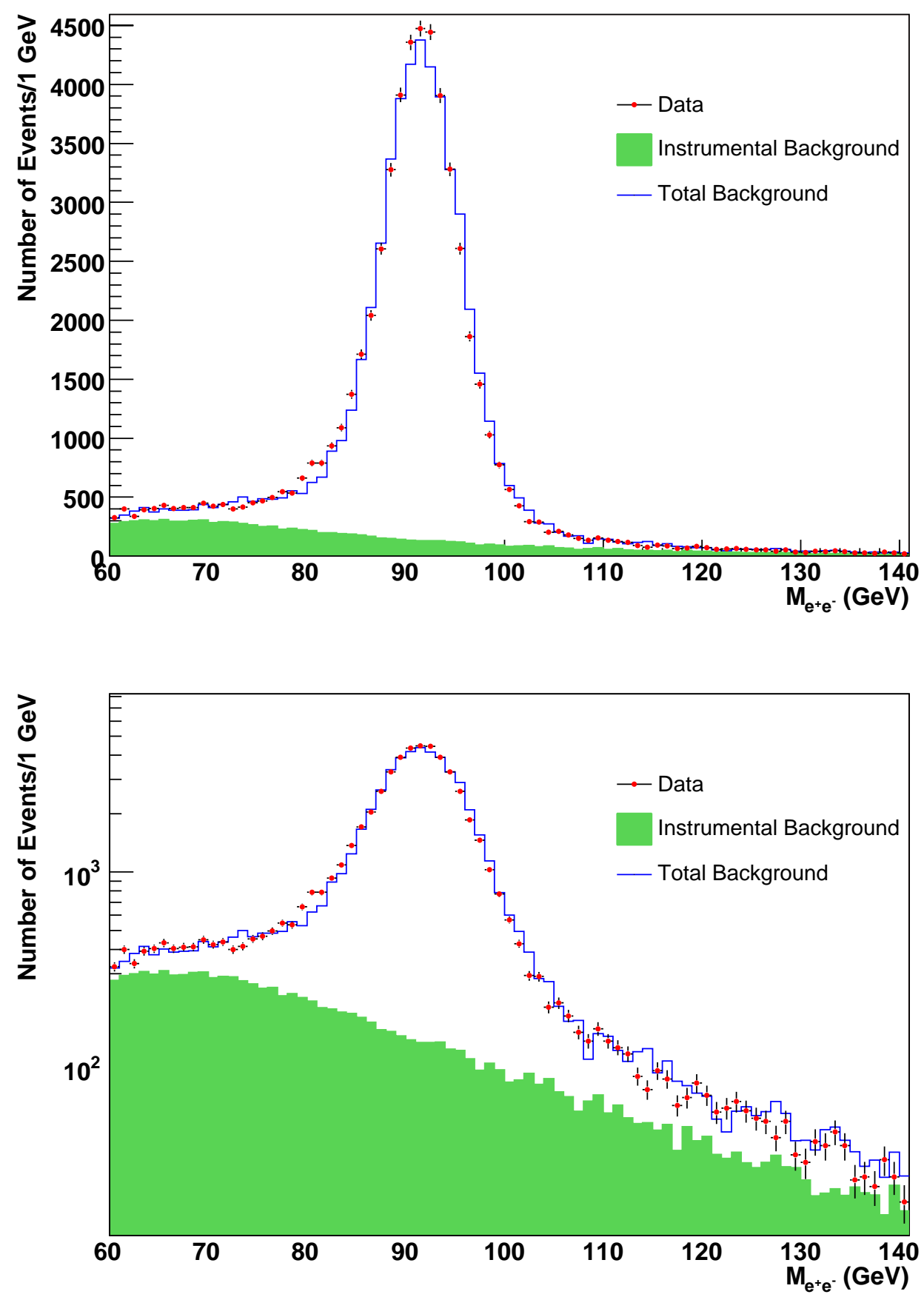

Figure 6.10: Data/Background comparison of invariant mass distribution for CCCC around $Z$ peak, combining "both in fiducial" and "one in fiducial" sub-sets. The upper plot is in linear scale, and the bottom is in logarithmic. 
for the ECCC is smaller than the normalization factors for instrumental background in the CCCC, as expected since the central electron must have an associated track and the forward electron must satisfy tighter selection criteria.

Adding the contributions of the background bin-by-bin, for CCCC and ECCC a good agreement around the $Z$ peak for the whole dataset is observed (Figure 6.11). The distribution for the ECCC sub-sample can be found in Appendix A.

The DY cross section is consistent within errors with what is expected from the measured $Z$ production cross-section $(264.9 \pm 3.9$ (stat) \pm 8.5 (sys) \pm 5.1 (pdf) \pm 17.2 (lumi) pb [80]).

\subsection{Z' Signal Monte Carlo Studies}

The expected shape and properties of $\mathrm{Z}^{\prime}$ signal were studied using Monte Carlo simulations. Sequential Z' $\rightarrow$ ee samples were generated using PYTHIA [73], processed through the standard D0 detector simulation, and passed through the same reconstruction chain as data events. Masses of $\mathrm{Z}^{\prime}$ bosons from $400-1000 \mathrm{GeV}$ were generated, with only the $\mathrm{Z}^{\prime}$ production being turned on at the generator level. The physics process $p \bar{p} \rightarrow \mathrm{Z}^{\prime} \rightarrow e e$ is the same for $Z$ and Drell-Yan. Since the actual process is $p \bar{p} \rightarrow \mathrm{Z}^{\prime} / Z / \gamma^{*} \rightarrow e e$, there can be interference between the $\mathrm{Z}^{\prime}$ and $Z / \gamma^{*}$. However, the effect of interference is negligible in the direct search for $\mathrm{Z}^{\prime}$ given the current experimental constraints on the $Z^{\prime}$ mass and the $Z^{\prime}-Z$ mixing. Furthermore, only the shape of the $\mathrm{Z}^{\prime}$ signal is used in setting the limit. In previous analyses it has been shown that $Z^{\prime}$ signals generated with interference off are consistent to the shape

of those $Z^{\prime}$ signals generated with the interference effects included when the $Z$ and the DY spectrum are subtracted from the combined spectrum. It has been shown 

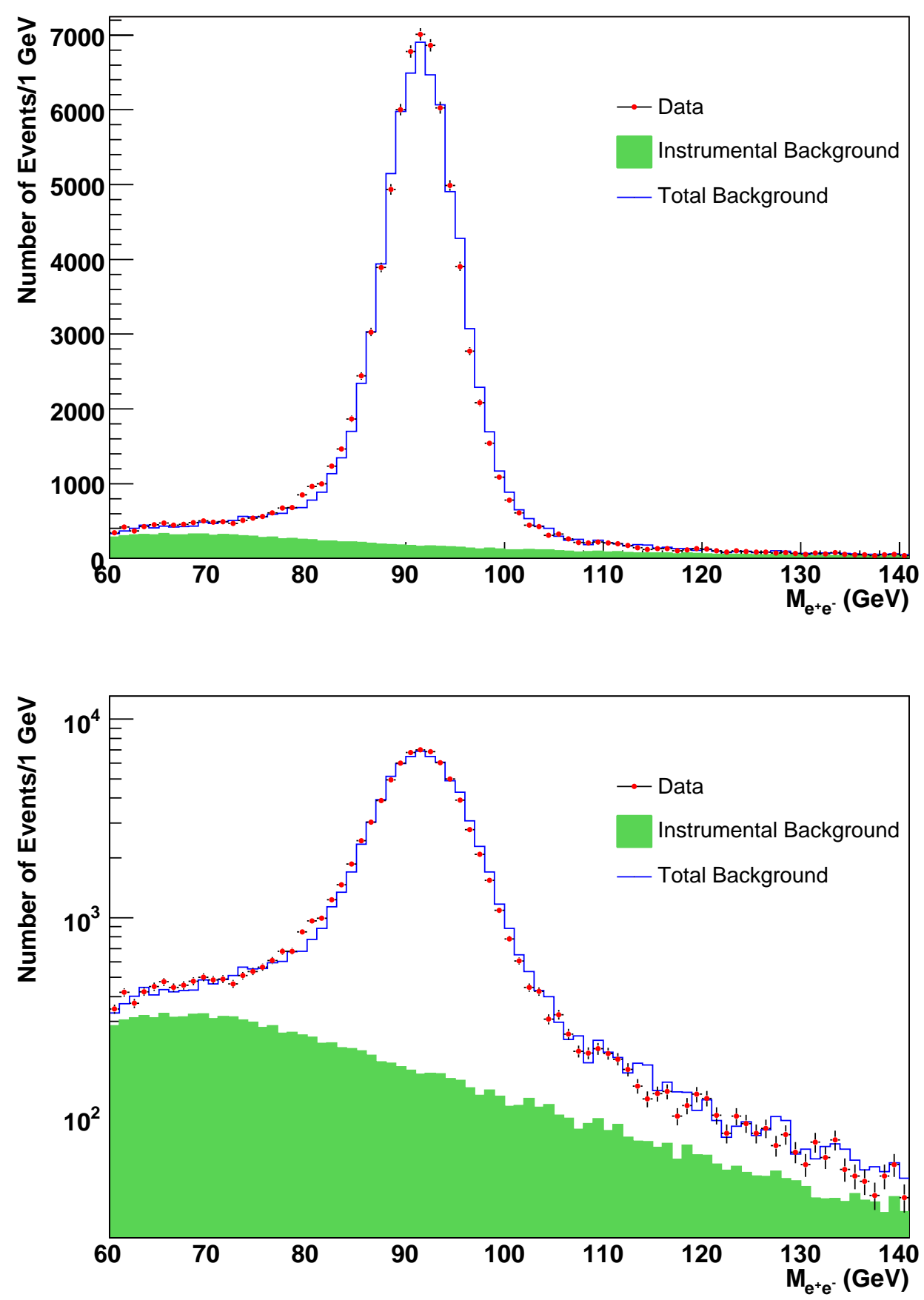

Figure 6.11: Data/Background comparison of invariant mass distribution around $Z$ peak, combining CCCC and ECCC topologies. The upper plot is in linear scale, and the bottom is in logarithmic. 


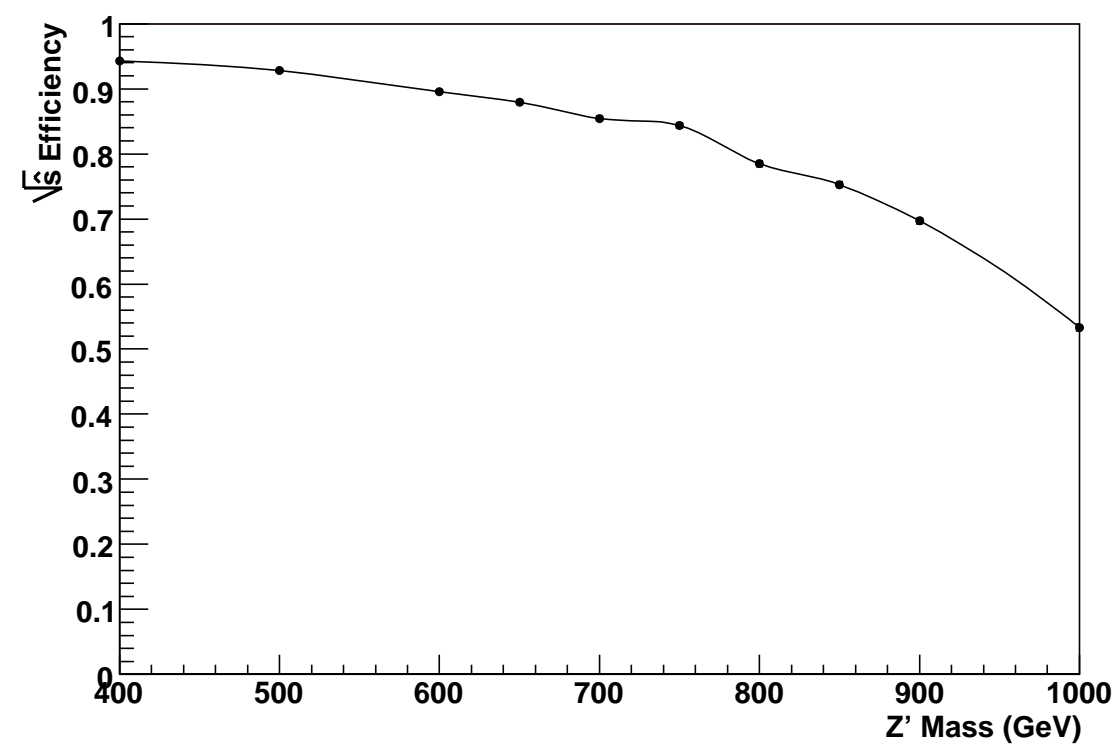

Figure 6.12: Efficiency of the $\sqrt{\hat{s}}$ cut, as a function of the $Z^{\prime}$ mass supplied as input to PYTHIA.

in previous studies [22] that the effect of the interference on the mass limit is at the level of $0.2 \%$ only, and is therefore neglected.

Simulated events are accepted for $\mathrm{Z}^{\prime}$ bosons produced with $\sqrt{\hat{s}}>\left[M_{Z^{\prime}}-3 \sigma\right]$, where $\sigma$ is the width of a Gaussian fit to the reconstructed $\mathrm{Z}^{\prime}$ peak. This selection is applied due to the fact that PYTHIA produces events with $Z^{\prime}$ masses as low as ten times the theoretical width below the Breit-Wigner pole value [81]. Such events end up in the low mass tail of the invariant mass spectrum and would not contribute to the resonance signal. The efficiency of the $\sqrt{\hat{s}}$ selection cut varies from $\sim 0.95$ for $400 \mathrm{GeV}$ to $\sim 0.5$ for $1000 \mathrm{GeV}$, and is shown in Figure 6.12 as a function of $\mathrm{Z}^{\prime}$ mass.

The reconstructed $\mathrm{MC}$ events are required to pass the same selection criteria as the data. For each of the MC generated $\mathrm{Z}^{\prime}$ data sets, the reconstructed ee invariant 
mass spectrum was fitted with a Gaussian to determine the signal reconstruction efficiency as well as the reconstructed mass and effective resolution. The results from these fits are summarized in Table 6.3, and Figure 6.13 shows the peak and the width of the Gaussian fit as a function of the generated Z' mass. Fits with Breit-Wigners convoluted with Gaussians were also performed but do not significantly change the results. The difference between the Gaussian and the Breit-Wigner fits is included in the systematic error. Figure 6.14 shows an example of the reconstructed invariant mass distribution fitted with a Gaussian for a $\mathrm{Z}^{\prime}$ of $850 \mathrm{GeV}$. Distributions for all the mass points used, can be found in Appendix B.

\begin{tabular}{|ccc|}
\hline $\begin{array}{c}\text { Generated Mass } \\
(\mathrm{GeV})\end{array}$ & $\begin{array}{c}\text { Fitted Mass } \\
(\mathrm{GeV})\end{array}$ & Fitted Sigma \\
\hline \hline 400 & $398.5 \pm 0.3$ & $19.3 \pm 0.3$ \\
500 & $498.9 \pm 0.4$ & $23.0 \pm 0.4$ \\
600 & $597.5 \pm 0.5$ & $26.6 \pm 0.5$ \\
650 & $646.4 \pm 0.5$ & $29.3 \pm 0.5$ \\
700 & $697.8 \pm 0.6$ & $31.7 \pm 0.6$ \\
750 & $744.5 \pm 0.6$ & $34.8 \pm 0.7$ \\
800 & $793.9 \pm 0.7$ & $36.7 \pm 0.7$ \\
850 & $843.7 \pm 0.7$ & $38.5 \pm 0.7$ \\
900 & $892.6 \pm 0.8$ & $40.6 \pm 0.8$ \\
1000 & $985.0 \pm 1.2$ & $47.4 \pm 1.0$ \\
\hline
\end{tabular}

Table 6.3: Results of Gaussian fits to the various Z' MC data sets. The errors listed correspond to the fit errors.

For each $\mathrm{Z}^{\prime} \mathrm{MC}$ data set, the geometric acceptance is defined by examining the generator level information and determining the fraction of MC generated events for which the two decay electrons hit each topology considered. The geometric acceptance varies from $\sim 0.5$ for $400 \mathrm{GeV}$ and rises up to $\sim 0.65$ for $1000 \mathrm{GeV}$ in $\mathrm{CCCC}$, and from $\sim 0.18$ for $400 \mathrm{GeV}$ to $\sim 0.05$ for $1000 \mathrm{GeV}$ in ECCC. The distribution of the 

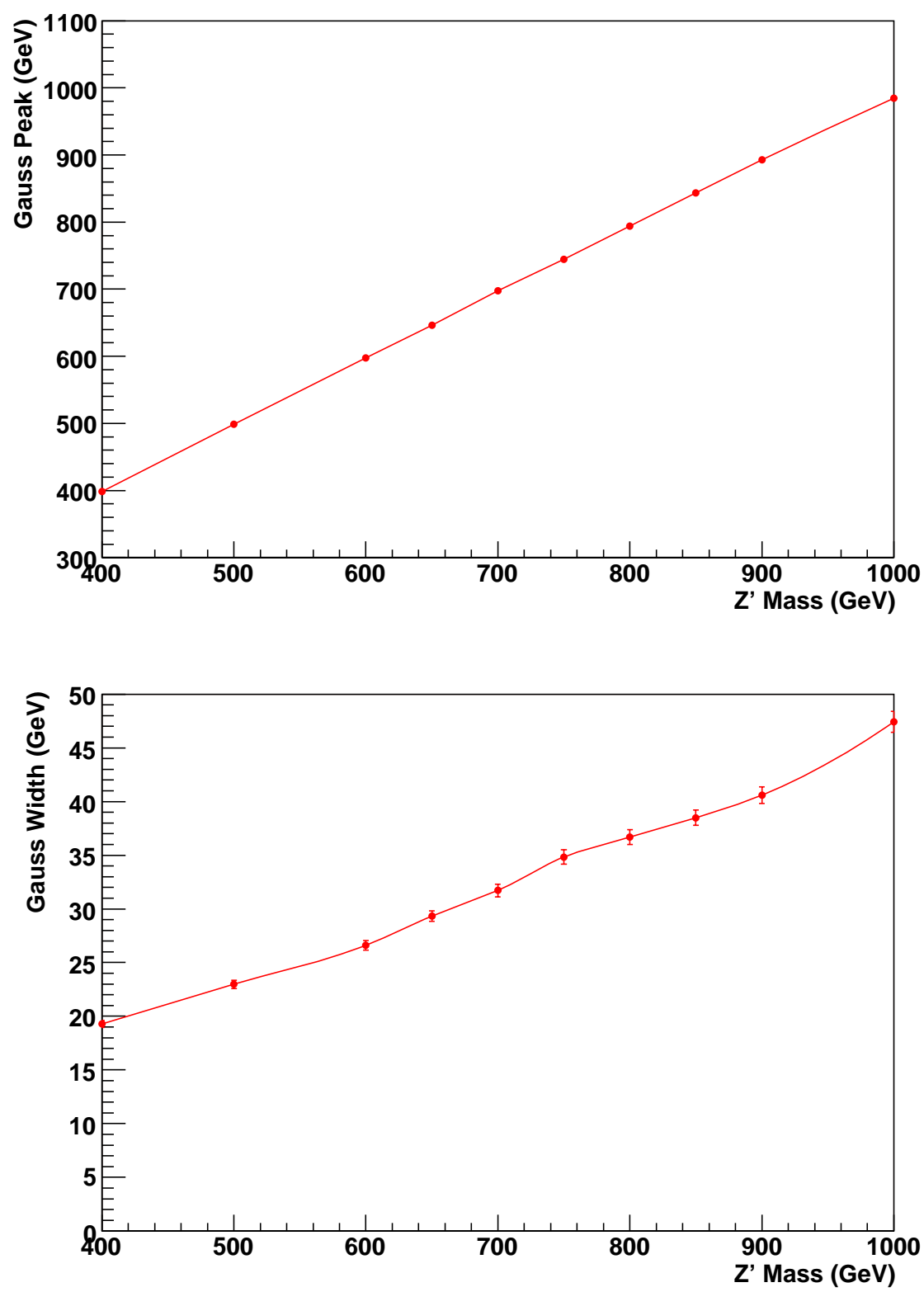

Figure 6.13: Results of the Gaussian fit applied to $\mathrm{Z}^{\prime}$ signal as a function of the generated $\mathrm{Z}^{\prime}$ mass. The upper plot shows the peak, and the bottom shows the width of the Gaussian, respectively. The error bars correspond to the fit error. 


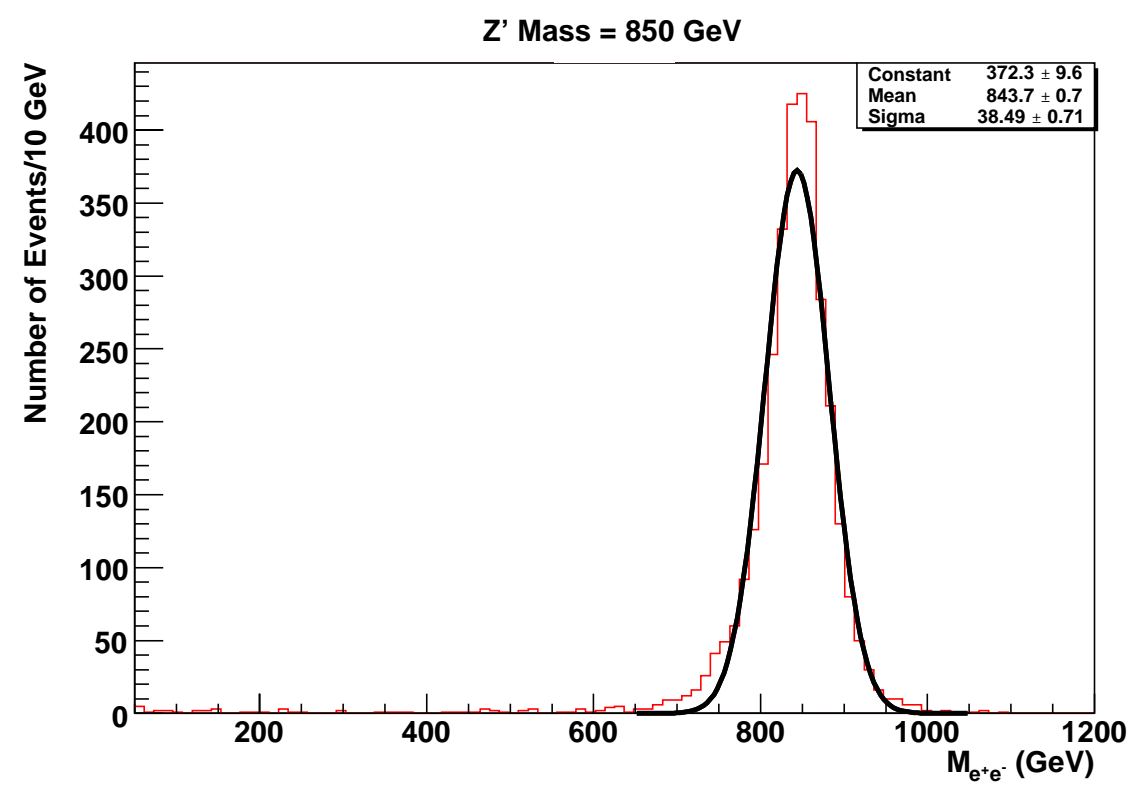

Figure 6.14: Reconstructed MC invariant mass distribution for a potential Z' $850 \mathrm{GeV}$ signal fitted with a Gaussian.

geometric acceptance as a function of the $\mathrm{Z}^{\prime}$ mass is shown in the upper plot of Figure 6.15.

The efficiency is defined as the fraction of MC events within each sub-sample which end up with the reconstructed ee mass within a search mass window. The efficiency includes the trigger efficiency, the object identification efficiencies, effects of the selection cuts, and the effect of the mass window. The efficiency for CCCC is about $\sim 0.85$, and for ECCC varies between $\sim 0.6$ and $\sim 0.4$. In Figure 6.15 is shown the efficiency for each sub-sample for an asymmetric window from $3 \sigma$ below the expected resonance mass, up to infinity. The total acceptance, that is the product of the geometric acceptance and the efficiency, varies between $\sim 0.4$ to $\sim 0.5$ for CCCC, and $\sim 0.1$ to $\sim 0.02$ for ECCC (Figure 6.16). Linear fits were performed 

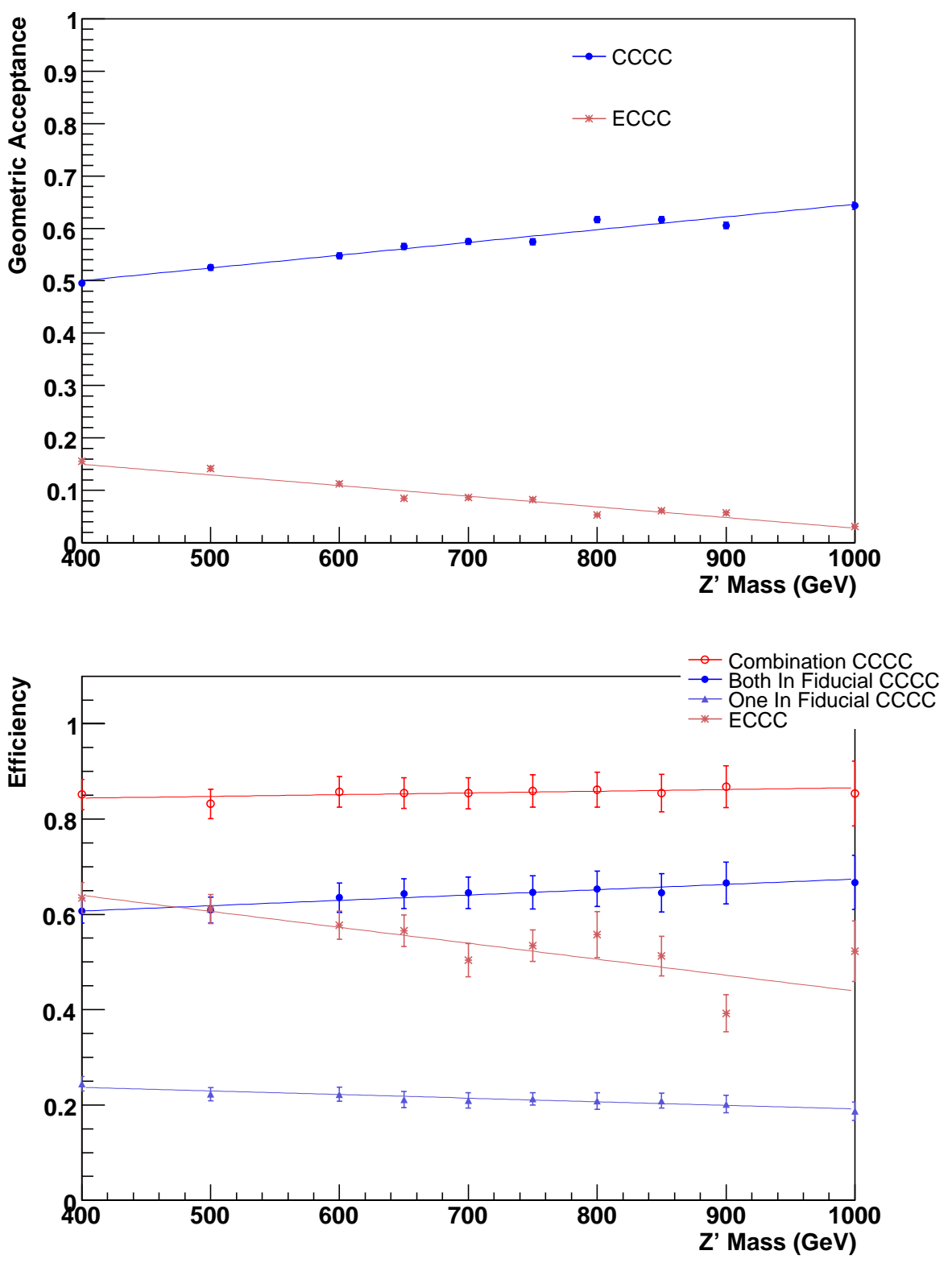

Figure 6.15: Geometric acceptance (upper plot) and Efficiency for each subsample used (bottom plot), for each $\mathrm{Z}^{\prime}$ mass point in an asymmetric window from $-3 \sigma$ to infinity. 


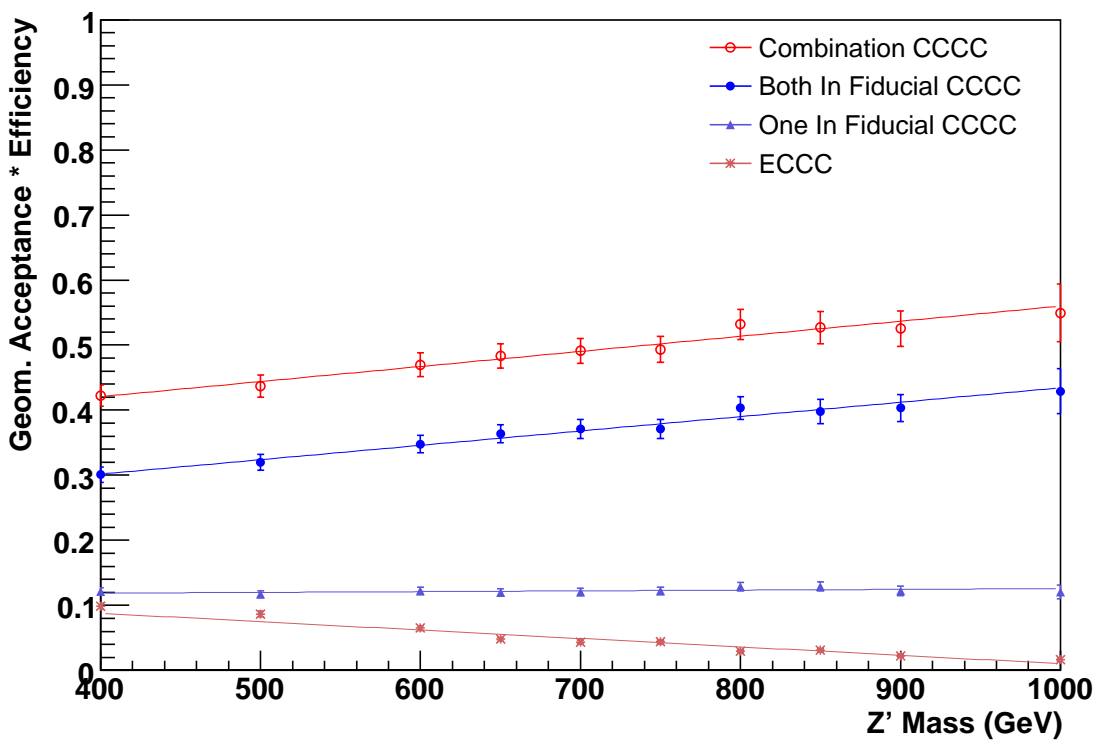

Figure 6.16: Total acceptance for each sub-sample, for each $\mathrm{Z}^{\prime}$ mass point in an asymmetric window from $-3 \sigma$ to infinity.

for each sub-sample for the geometric acceptance, efficiency, and total acceptance to demonstrate the behavior as a function of the generated mass. For the analysis, the actual values of the geometric acceptance, efficiency and total acceptance were used, except for the case of the $900 \mathrm{GeV}$ efficiency that the fit value was used.

To quantify the results of the search for a resonant $\mathrm{Z}^{\prime}$ production, the numbers of measured and expected background events will be counted within a mass window defined around each input mass. The expected efficiency is examined for various mass windows, including symmetric windows varying from $\pm 1 \sigma$ up to $\pm 4 \sigma$. The efficiencies are very similar for windows which are at least $\approx \pm 3 \sigma$ wide. The final choice is made to use an asymmetric window from $3 \sigma$ below the expected resonance mass, up to infinity, in order to avoid issues with the $\sqrt{\hat{s}}$ selection applied to the generated events 
while maintaining excellent sensitivity.

\subsection{Extrapolating to Higher Invariant Masses}

Once the background has been normalized around the $Z$ peak, the normalization factors are used to extrapolate to higher invariant masses. Figure 6.17 shows the full range of the invariant mass spectrum studied for the combined CCCC sample. Table 6.4 compares the observed number of data events to the expected background events that have $M_{e e}$ above a given lower limit $M$. This table and the subsequent similar ones contain the expected number of events, its uncorrelated error that combines the systematic error components that are uncorrelated and the statistical error that is the combination of the MC statistical error of the DY contribution and the QCD contribution, and the error that is a result of the correlated error components. Systematic errors are discussed in more detail in Section 7.3.1. The plots for each individual sub-set can be found in Appendix A, and the tables in Appendix C.

Combining the two topologies no significant excess is observed (see Figure 6.18 upper plot, and Table 6.5 for the comparison of number of data events to the expected background events). A limit of the cross section times the branching ratio will therefore be set. Should an $850 \mathrm{GeV} Z^{\prime}$ existed, it would give us a signal of about 8 events and would clearly be seen (see Figure 6.18 bottom plot). 


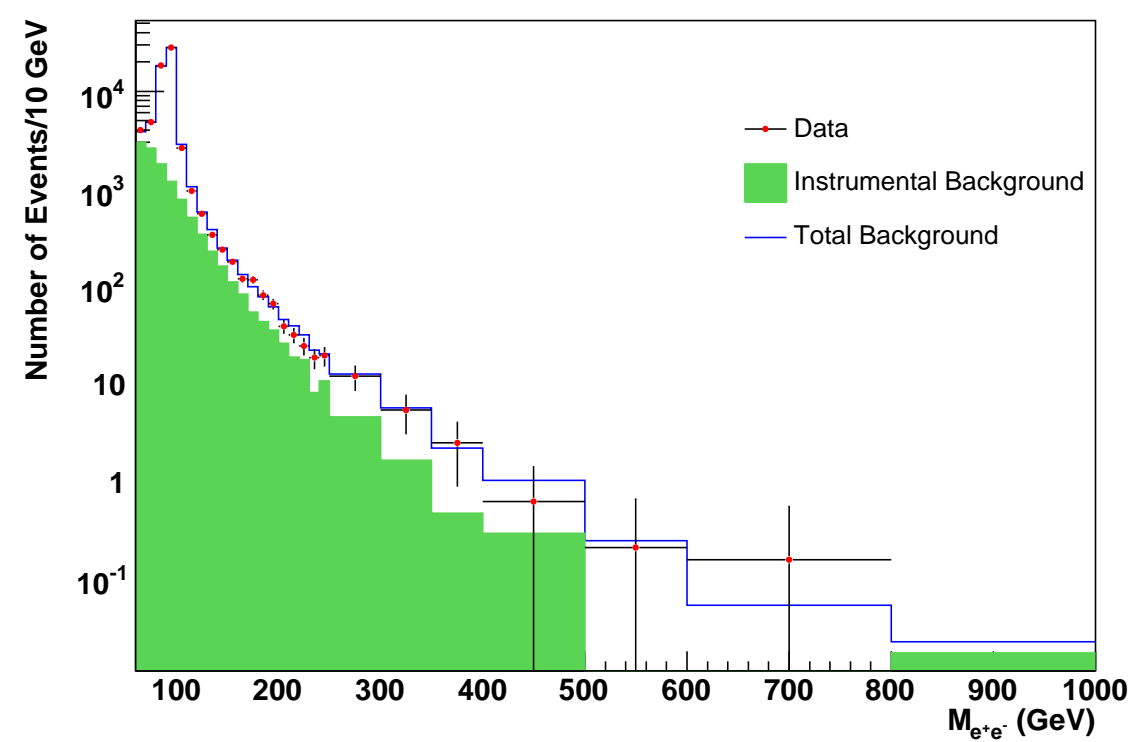

Figure 6.17: Data/Background comparison of the full range invariant mass distribution in CCCC, combining "both in fiducial" and "one in fiducial" sub-sets. 


\begin{tabular}{|ccc|}
\hline $\begin{array}{c}\text { Mass } \\
(\mathrm{GeV})\end{array}$ & $\begin{array}{c}\text { Data } \\
\text { Events }\end{array}$ & $\begin{array}{c}\text { Expected Background } \\
\text { Events }\end{array}$ \\
\hline \hline 60 & 60977 & $61082 \pm 455 \pm 5344$ \\
80 & 52170 & $52342 \pm 413 \pm 4579$ \\
100 & 5458 & $5918 \pm 148 \pm 518$ \\
150 & 789 & $819 \pm 13 \pm 72$ \\
200 & 237 & $270 \pm 7 \pm 24$ \\
250 & 107 & $113 \pm 5 \pm 10$ \\
300 & 49 & $52.0 \pm 2.6 \pm 4.6$ \\
350 & 23 & $24.3 \pm 1.2 \pm 2.1$ \\
400 & 11 & $13.73 \pm 0.73 \pm 1.20$ \\
450 & 6 & $7.27 \pm 0.44 \pm 0.64$ \\
500 & 5 & $3.80 \pm 0.21 \pm 0.33$ \\
550 & 4 & $2.31 \pm 0.12 \pm 0.20$ \\
600 & 3 & $1.459 \pm 0.073 \pm 0.128$ \\
650 & 2 & $0.985 \pm 0.045 \pm 0.086$ \\
700 & 0 & $0.698 \pm 0.029 \pm 0.061$ \\
750 & 0 & $0.533 \pm 0.020 \pm 0.047$ \\
800 & 0 & $0.444 \pm 0.016 \pm 0.039$ \\
850 & 0 & $0.0608 \pm 0.0060 \pm 0.0053$ \\
900 & 0 & $0.0314 \pm 0.0039 \pm 0.0028$ \\
950 & 0 & $0.0162 \pm 0.0027 \pm 0.0014$ \\
1000 & 0 & $0.0080 \pm 0.0018 \pm 0.0007$ \\
\hline
\end{tabular}

Table 6.4: Number of events above mass for combined CCCC topology. 


\begin{tabular}{|ccccc|}
\hline $\begin{array}{c}\text { Mass } \\
(\mathrm{GeV})\end{array}$ & $\begin{array}{c}\text { Data } \\
\text { Events }\end{array}$ & \multicolumn{3}{c|}{ Expected Background } \\
Events
\end{tabular}

Table 6.5: Number of events above mass for combined CCCC and ECCC topologies. 

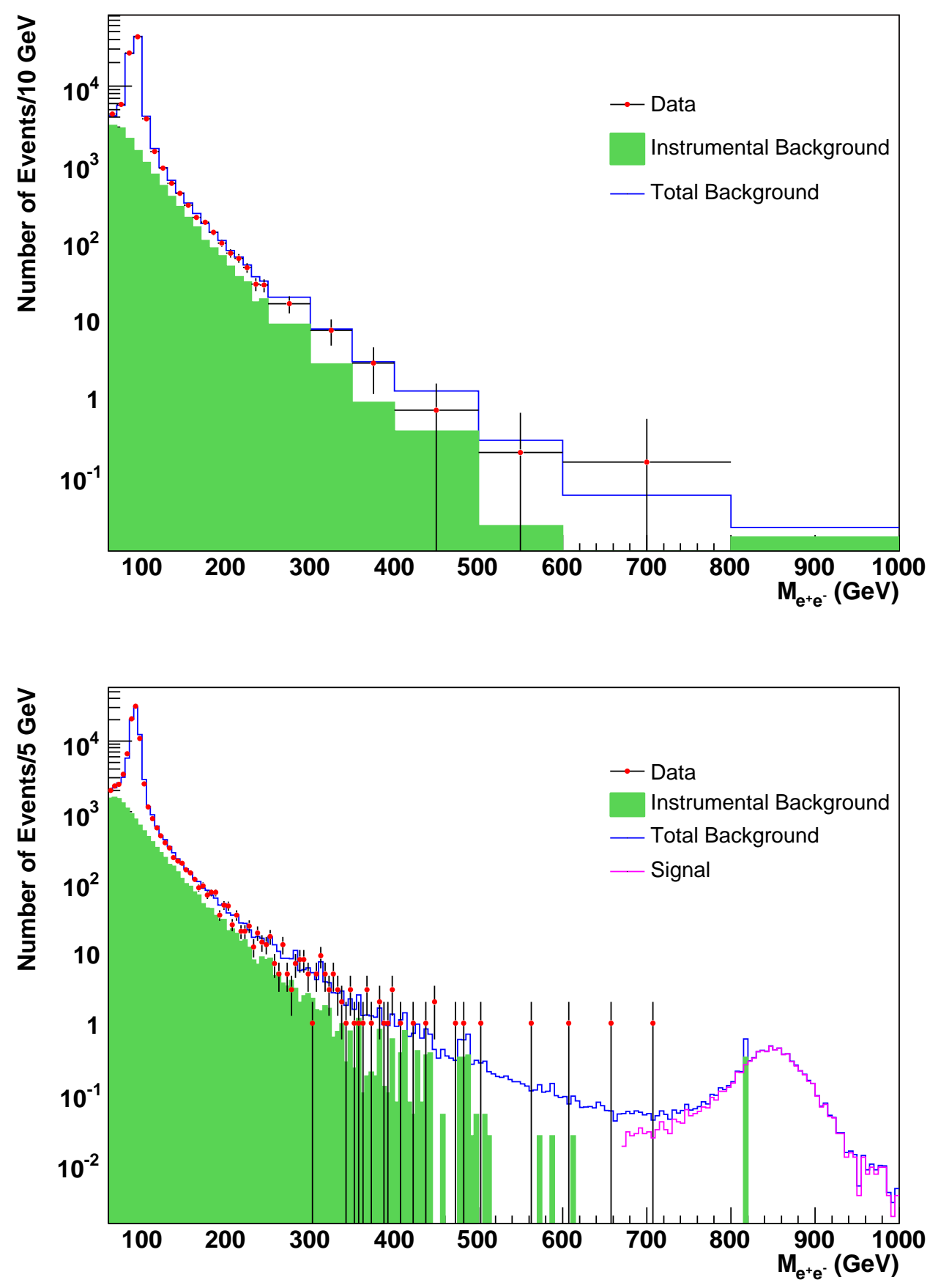

Figure 6.18: Data/Background comparison of Invariant Mass distribution extrapolated to high masses, combining CCCC and ECCC topologies. The bottom plot shows how a potential $850 \mathrm{GeV}$, signal normalized to the theoretical cross-section, would appear. 


\section{Chapter 7}

\section{Extraction of Limit}

\section{$7.1 \quad$ Introduction}

No significant excess of events, compatible to what would be expected from the decay of a narrow resonance like $\mathrm{Z}^{\prime}$, is observed in the dielectron mass spectrum. In the absence of a $Z^{\prime}$ signal, an upper limit on the product of the cross section times the branching ratio $\left(\sigma\left(p \bar{p} \rightarrow \mathrm{Z}^{\prime}\right) \times B R\left(\mathrm{Z}^{\prime} \rightarrow e e\right)\right)$ is set. For the remaining of this chapter whenever the cross section is mentioned, the product of the cross section times the branching ratio is implied. A Bayesian approach is used to set a limit, according to the recipe adopted by D0 $[82,83]$. 


\subsection{Bayesian Technique}

In a counting experiment, like in searches for new particles, the expected number of events $\mu$ is related to the signal cross section times branching ratio $\sigma \times B R$ as:

$$
\mu=b+\mathcal{L} \varepsilon(\sigma \times B R)
$$

where $\mathcal{L}$ is the integrated luminosity, $b$ the expected background, and $\varepsilon$ the total signal acceptance. The probability of observing $N_{W}$ events in a mass window around the $\mathrm{Z}^{\prime}$ peak can be described by a Poisson distribution:

$$
P\left(N_{W} \mid \mu\right)=\frac{e^{-\mu} \mu^{N_{W}}}{N_{W} !}
$$

When substituting Equation 7.1 into Equation 7.2, the probability becomes:

$$
P\left(N_{W} \mid \sigma \times B R, \mathcal{L}, \varepsilon, b\right)=\frac{e^{-(b+\mathcal{L} \varepsilon \sigma \times B R)}(b+\mathcal{L} \varepsilon \sigma \times B R)^{N_{W}}}{N_{W} !}
$$

Bayes' theorem relates the posterior probability (post-data knowledge of the parameters) to the product of the prior probability (pre-data knowledge) and the likelihood [84], as:

$$
\text { posterior }=\frac{\text { likelihood } \times \text { prior }}{\text { normalization factor }}
$$

or formally as:

$$
P(A \mid B)=\frac{P(B \mid A) P(A)}{P(B)}
$$

where, $P(A)$ is the prior probability, $P(A \mid B)$ is the posterior probability or the probability of $A$ given $B$ is true, $P(B \mid A)$ is the conditional probability of $\mathrm{B}$ given $\mathrm{A}$, and $P(B)$ is the prior of $\mathrm{B}$ and is the normalizing factor. 
In case there are more than two variables, Equation 7.4 becomes:

$$
P(A \mid B C)=\frac{P(B \mid A C) P(A \mid C)}{P(B \mid C)}
$$

where the normalization factor is determined by:

$$
\sum_{\text {allA }} P(A \mid B C)=1
$$

Adapting Equations 7.5 and 7.6 to this study,

- $A$ corresponds to the probability of the cross section being between $\sigma$ and $\sigma+d \sigma$, the integrated luminosity between $\mathcal{L}$ and $\mathcal{L}+d \mathcal{L}$, the total signal acceptance between $\varepsilon$ and $\varepsilon+d \varepsilon$, and the background events between $b$ and $b+d b$ in a mass window,

- $B$ corresponds to the $N_{W}$ data events observed in the same mass window,

- $C$ corresponds to all relevant "prior knowledge" $\lambda$. This includes the descriptions of the knowledge of the parameters $\sigma, \mathcal{L}, \varepsilon$, and $b$.

Hence, Bayes' theorem becomes:

$P\left(\sigma \times B R, \mathcal{L}, \varepsilon, b \mid N_{W}, \lambda\right)=\mathrm{constant} \times \frac{e^{-(b+\mathcal{L} \varepsilon \sigma \times B R)}(b+\mathcal{L} \varepsilon \sigma \times B R)^{N_{W}}}{N_{W} !} P(\sigma \times B R \mid \lambda) P(\mathcal{L}, \varepsilon, b \mid \lambda)$

where the constant is determined by:

$$
\int_{0}^{\infty} d \sigma \int_{0}^{\infty} d \mathcal{L} \int_{0}^{1} d \varepsilon \int_{0}^{\infty} d b \rho\left(\sigma \times B R, \mathcal{L}, \varepsilon, b \mid N_{W}, \lambda\right)=1
$$

Since the quantity of interest is $\sigma \times B R$, the dependence on parameters $\mathcal{L}, \varepsilon$, and $b$ 
can be removed by integrating over them. Then the posterior probability distribution for $\sigma \times B R$ becomes:

$$
\rho\left(\sigma \times B R \mid N_{W}, \lambda\right)=\int_{0}^{\infty} d \mathcal{L} \int_{0}^{1} d \varepsilon \int_{0}^{\infty} d b \rho\left(\sigma \times t o B R, \mathcal{L}, \varepsilon, b \mid N_{W}, \lambda\right)
$$

The relevant quantity to be calculated is the upper limit on the cross section times the branching ratio $\left(\sigma^{U L}\right)$ at some confidence level. This upper limit is obtained by solving:

$$
C L=\int_{0}^{\sigma^{U L}} d \sigma \rho\left(\sigma \times B R \mid N_{W}, \lambda\right)
$$

where $C L$ is the desired confidence level. The selected confidence level is $95 \%$.

\subsection{Inputs to Limit Calculator}

The D0 limit calculator [85] is used to calculate the upper limit on $\sigma \times B R$ at the 95\% confidence level. The inputs to the limit calculator for a given $\mathrm{Z}^{\prime}$ mass are:

- $N_{W}$, the number of observed data events in a mass window

- $b$, the number of expected background events in the mass window

- $\varepsilon$, the total signal acceptance

- $\mathcal{L}$, the integrated luminosity

- the uncertainties on $b, \varepsilon$, and $\mathcal{L}$.

With the above inputs to the calculator, there are two possible values that can be calculated; the "observed" upper limit, where all of the above inputs are used, and 
the "expected" upper limit where the number of expected background events in the mass window $b$ is used in place of the observed data events $N_{W}$. Thus, the expected limit expresses the expected sensitivity.

A discussion about the various systematic uncertainties follows, and then all the inputs to the limit calculator will be summarized.

\subsubsection{Systematic Uncertainties}

The systematic uncertainties are separated into "correlated" errors which are correlated for all sub-samples, and "uncorrelated" ones. The correlated errors are due to the trigger, object identification, higher order corrections, and PDFs. The uncorrelated ones are due to the normalization factor for misidentified electrons, correction of non-fiducial electrons, correction of end-cap electrons, reweighting of the $Z p_{T}$ spectrum, and the signal fit function. Errors due to the integrated luminosity measurement are also correlated for all the samples.

\section{Luminosity}

The uncertainty on the measured integrated luminosity for Run IIa is assigned by the D0 experiment to be $\pm 6.1 \%$ [49]. This includes fit uncertainties in the analytic correction functions derived from luminosity measurements and an estimate of the uncertainty associated with the effect of scintillator radiation damage.

\section{Trigger}

The trigger efficiency for high $p_{\mathrm{T}}$ electrons has been calculated to be $0.995 \pm 0.002$ [86]. 


\section{Object Identification}

Electron efficiency correction factors are applied to account for differences between data and MC in the efficiencies of all the selection requirements. The selection efficiencies are measured for events around the $Z$ peak, using the tag and probe method that was described in Section 5.4. More specifically, the probe needs to satisfy the quality requirements that the efficiency is measured, and the tag electron needs to satisfy the following more strigent selection criteria:

- $f_{\mathrm{EM}} \geq 0.9$

- $f_{\text {iso }} \leq 0.2$

- $\mathrm{H}-\operatorname{Matrix}(7) \leq 50$

- Spatial track-match $\chi^{2} \geq 0.0$

- Likelihood $\geq 0.85$

- $p_{\mathrm{T}} \geq 15.0 \mathrm{GeV}$

- A single-electron trigger within a cone with $\Delta R \leq 0.4$.

The same method is applied to both data and MC, and the ratio is considered. This ratio suffers from uncertainty that the maximum occurs at high $\eta$ - regions. For the region considered in this analysis, the maximum uncertainty of $2.5 \%$ per electron is considered as a conservative estimate $[70,86]$.

\section{Misidentified Electrons}

As described in Section 6.4, two different fit ranges were used in normalizing the background to the data. The resultant difference in expected background events is 
considered as a systematic error. This error ranges from $0.2 \%$ for lower mass values to $1.5 \%$ for higher masses. For $900 \mathrm{GeV}$ this error is about $\pm 1.4 \%$.

\section{Correction of Non-Fiducial Electrons}

As described in Section 6.3.1, two fit functions were considered to correct the nonfiducial electrons of the CC. The expected background events for each different case were calculated and the difference was set as a systematic error. This error ranges from $\pm 1.4 \%$ for lower masses to $\pm 0.2 \%$ for higher masses. For $900 \mathrm{GeV}$ this error is about $\pm 0.2 \%$.

\section{Correction of End-Cap Electrons}

The difference in expected background events when the correction to EC electrons is applied, as compared to no correction applied is considered as a systematic error. This error ranges from $\pm 8 \%$ for lower masses to $\pm 1 \%$ for higher masses. For $900 \mathrm{GeV}$ this error is about $\pm 1.4 \%$.

\section{Reweighting of the $Z p_{T}$ Spectrum}

To correct the $p_{T}$ distribution of the $Z$ boson, a set of weights that was the average between the D0 weights and the case of no correction, was applied. The difference in expected background events is considered as a systematic error. This error ranges from $0.5 \%$ for lower masses to $7 \%$ for higher masses. For $900 \mathrm{GeV}$ this error is about $\pm 7 \%$. 


\section{Higher Order Corrections}

To compensate for the NLO effect a mass independent $k$-factor of 1.3 has been used, the same as for the Drell-Yan process. This is a reasonable assumption because the signal and the Drell-Yan process differ only in their out-going leptons. However, the $k$-factor has a slight mass dependence and this dependency is treated as a source of uncertainty. Figure 7.1 shows the mass dependence of the $k$-factor for NLO and NNLO [87]. The region above $400 \mathrm{GeV}$ is covered by a band that extends $\pm 8.0 \%$ around the used $k$-factor and this is the uncertainty used.

\section{Parton Density Functions}

Uncertainties due to the PDF's affect the theoretical production cross sections, and the efficiency for the signal. The uncertainties on the signal cross section are combined with the uncertainties on the $k$-factor discussed in the following section.

To determine the uncertainties on the signal efficiency, information delivered by a central D0 tool called "pdf_reweighting" [88] is used. This processor reweights the events from the leading order pdf set CTEQ6L [13] to the next-to-leading order pdf set CTEQ6M [89] with respect to their Bjorken-x. For every incoming proton and anti-proton $2 \times 20$ error functions are determined. The efficiency deviation from every of the $2 \times 20$ error functions to CTEQ6M is determined. If $B$ is the central value for CTEQ6M and $B_{i}$ is the value for each one of the $2 \times 20$ error functions, then the deviation $B-B_{i}$ is calculated for each one. If $\left(B-B_{i}\right)>0$ then the error is called "positive", and when $\left(B-B_{i}\right)<0$ the error is called "negative". The quadratical summation of all "positive" ("negative") differences yields the "positive" ("negative") efficiency uncertainty for the signal. The larger of the two uncertainties is considered. Results for each Z' mass are listed in Table 7.1. 


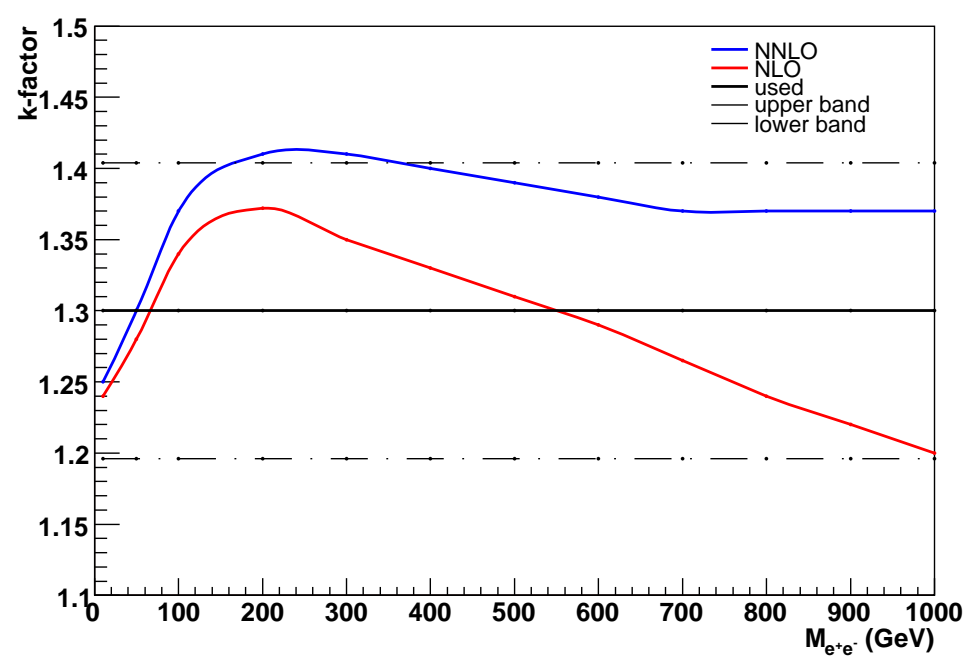

Figure 7.1: NLO and NNLO $k$-factors for a sequential $\mathrm{Z}^{\prime}$ as a function of the invariant mass of the electron pair [87]. Also shown is the mass-independent value $k=1.3$ used in this analysis, and the upper and lower band considered as a systematic error.

\begin{tabular}{|ccc|}
\hline $\begin{array}{c}\text { Mass } \\
(\mathrm{GeV})\end{array}$ & $\begin{array}{c}\text { Positive Uncertainty } \\
(\%)\end{array}$ & $\begin{array}{c}\text { Negative Uncertainty } \\
(\%)\end{array}$ \\
\hline \hline 400 & 0.28 & 0.21 \\
500 & 0.22 & 0.33 \\
600 & 0.34 & 0.63 \\
650 & 0.42 & 0.78 \\
700 & 0.62 & 1.01 \\
750 & 0.78 & 1.27 \\
800 & 1.30 & 2.09 \\
850 & 1.78 & 2.67 \\
900 & 2.54 & 3.48 \\
1000 & 5.40 & 7.06 \\
\hline
\end{tabular}

Table 7.1: Uncertainties of Z' signal efficiency due to PDFs for different mass points. 


\section{Signal Fit Function}

The reconstructed ee invariant mass spectrum for each of the MC generated $Z^{\prime}$ data sets, was fitted with two functions, a Gaussian, and a Breit-Wigner convoluted with a Gaussian. The difference in events inside an asymmetric window from $3 \sigma$ below the expected resonance mass up to infinity, produced by the two different fits is considered as a systematic error. This error ranges from $1.8 \%$ for lower masses up to $7 \%$ for higher masses. A typical value for a $\mathrm{Z}^{\prime}$ with generated mass of $900 \mathrm{GeV}$ is about $\pm 6 \%$.

\subsubsection{Luminosity}

The luminosity is calculated using the official D0 luminosity software package lm_tools [90] that interfaces with the database and retrieves information for each requested run, for events that have been marked as good and pass specific triggers. Table 7.2 provides the breakdown of the luminosity for the triggers used for the analysis. For events in the CCCC topology all the Run IIa data set is used, whereas for the ECCC topology data collected from December 2005 to February 2006 were ignored (cable-swap data). For the CCCC topology the integrated luminosity adds up to $(1106 \pm 67) \mathrm{pb}^{-1}$, and for the ECCC topology $(1007 \pm 61) \mathrm{pb}^{-1}$.

\subsubsection{Data and Background Events}

For an asymmetric window from $3 \sigma$ below a given $\mathrm{Z}^{\prime}$ mass up to infinity, the data, the expected background events and the associated total error where statistical and systematic contributions are added in quadrature, are counted and summarized in Tables 7.3, 7.4 and 7.5 for the combined CCCC, ECCC, and the combined CCCC - 


\begin{tabular}{|cc|c|c|}
\hline \multirow{2}{*}{$\begin{array}{c}\text { Trigger } \\
\text { Name }\end{array}$} & $\begin{array}{c}\text { Trigger } \\
\text { Version }\end{array}$ & \multicolumn{2}{|c|}{ Integrated Luminosity $\left(p b^{-1}\right)$} \\
\cline { 3 - 4 } & CCCC & ECCC \\
\hline \hline EM_MX & V8.0 - V9.0 & 27.04 & 27.04 \\
EM_MX & V9.0 - V10.0 & 36.23 & 36.23 \\
EM_MX & V10.0 - V11.0 & 10.98 & 10.98 \\
EM_MX & V11.0 - V12.0 & 65.95 & 65.95 \\
E1_SH30 & V12.0 - V13.0 & 240.08 & 240.08 \\
E1_SHT22 & V13.0 - V13.3 & 57.60 & 57.60 \\
E1_SHT22 & V13.3 - V14.0 & 326.66 & 326.66 \\
E1_SHT25 & V14.0 - V15.0 & 341.28 & 242.35 \\
\hline
\end{tabular}

Table 7.2: Breakdown of the integrated luminosity according to trigger version.

ECCC topologies, respectively. Tables of the individual CCCC sub-samples can be found in Appendix C.

\subsubsection{Acceptance}

For the same asymmetric window from $3 \sigma$ below a given $\mathrm{Z}^{\prime}$ mass up to infinity, the different efficiencies and acceptances are calculated (as described in Section 6.5). The results are shown in Tables 7.6, and 7.7 for the combined CCCC topology, and ECCC topology, respectively. Table 7.8 shows the total Z' signal acceptance for the total combined CCCC and ECCC sample. The input in the limit calculator is the total acceptance, that is the product of the "Geometric Acceptance" $\times$ "Window Efficiency". The $\sqrt{\hat{s}}$ acceptance is absorbed into the theoretical cross section. 


\begin{tabular}{|cccc|}
\hline $\begin{array}{c}\text { Mass } \\
(\mathrm{GeV})\end{array}$ & $\begin{array}{c}\text { Lower } \\
\text { Mass Bound } \\
(\mathrm{GeV})\end{array}$ & $\begin{array}{c}\text { Data } \\
\text { Events }\end{array}$ & $\begin{array}{c}\text { Expected Background } \\
\text { Events }\end{array}$ \\
\hline \hline 400 & 342.1 & 24 & $26.6 \pm 2.7$ \\
500 & 431 & 8 & $9.20 \pm 0.92$ \\
600 & 520.2 & 4 & $3.09 \pm 0.32$ \\
650 & 562.1 & 4 & $2.07 \pm 0.21$ \\
700 & 604.9 & 3 & $1.41 \pm 0.14$ \\
750 & 645.6 & 2 & $1.03 \pm 0.10$ \\
800 & 689.9 & 1 & $0.745 \pm 0.072$ \\
850 & 734.5 & 0 & $0.580 \pm 0.055$ \\
900 & 778.2 & 0 & $0.484 \pm 0.046$ \\
1000 & 857.8 & 0 & $0.0570 \pm 0.0076$ \\
\hline
\end{tabular}

Table 7.3: Number of events in asymmetric mass window for CCCC topology.

\begin{tabular}{|cccc|}
\hline $\begin{array}{c}\text { Mass } \\
(\mathrm{GeV})\end{array}$ & $\begin{array}{c}\text { Lower } \\
\text { Mass Bound } \\
(\mathrm{GeV})\end{array}$ & $\begin{array}{c}\text { Data } \\
\text { Events }\end{array}$ & $\begin{array}{c}\text { Expected Background } \\
\text { Events }\end{array}$ \\
\hline \hline 400 & 342.1 & 5 & $7.87 \pm 1.00$ \\
500 & 431 & 1 & $1.72 \pm 0.22$ \\
600 & 520.2 & 0 & $0.395 \pm 0.049$ \\
650 & 562.1 & 0 & $0.269 \pm 0.036$ \\
700 & 604.9 & 0 & $0.120 \pm 0.016$ \\
750 & 645.6 & 0 & $0.0465 \pm 0.0066$ \\
800 & 689.9 & 0 & $0.0239 \pm 0.0038$ \\
850 & 734.5 & 0 & $0.0121 \pm 0.0023$ \\
900 & 778.2 & 0 & $0.0062 \pm 0.0015$ \\
1000 & 857.8 & 0 & $0.00188 \pm 0.00074$ \\
\hline
\end{tabular}

Table 7.4: Number of events in asymmetric mass window for ECCC topology. 


\begin{tabular}{|cccc|}
\hline $\begin{array}{c}\text { Mass } \\
(\mathrm{GeV})\end{array}$ & $\begin{array}{c}\text { Lower } \\
\text { Mass Bound } \\
(\mathrm{GeV})\end{array}$ & $\begin{array}{c}\text { Data } \\
\text { Events }\end{array}$ & $\begin{array}{c}\text { Expected Background } \\
\text { Events }\end{array}$ \\
\hline \hline 400 & 342.1 & 29 & $34.5 \pm 3.4$ \\
500 & 431 & 9 & $10.9 \pm 1.1$ \\
600 & 520.2 & 4 & $3.48 \pm 0.35$ \\
650 & 562.1 & 4 & $2.34 \pm 0.23$ \\
700 & 604.9 & 3 & $1.53 \pm 0.15$ \\
750 & 645.6 & 2 & $1.08 \pm 0.11$ \\
800 & 689.9 & 1 & $0.769 \pm 0.074$ \\
850 & 734.5 & 0 & $0.592 \pm 0.056$ \\
900 & 778.2 & 0 & $0.490 \pm 0.046$ \\
1000 & 857.8 & 0 & $0.0589 \pm 0.0077$ \\
\hline
\end{tabular}

Table 7.5: Number of events in asymmetric mass window for CCCC and ECCC topologies combined.

\begin{tabular}{|cccc|}
\hline $\begin{array}{c}\text { Mass } \\
(\mathrm{GeV})\end{array}$ & $\begin{array}{c}\text { Geometric } \\
\text { Acceptance }\end{array}$ & $\begin{array}{c}\text { Window } \\
\text { Efficiency }\end{array}$ & $\begin{array}{c}\text { Total } \\
\text { Acceptance }\end{array}$ \\
\hline \hline 400 & $0.4957 \pm 0.0054$ & $0.852 \pm 0.032$ & $0.422 \pm 0.016$ \\
500 & $0.5253 \pm 0.0054$ & $0.832 \pm 0.031$ & $0.437 \pm 0.017$ \\
600 & $0.5478 \pm 0.0056$ & $0.858 \pm 0.032$ & $0.470 \pm 0.018$ \\
650 & $0.5655 \pm 0.0055$ & $0.855 \pm 0.032$ & $0.483 \pm 0.019$ \\
700 & $0.5749 \pm 0.0054$ & $0.854 \pm 0.033$ & $0.491 \pm 0.019$ \\
750 & $0.5743 \pm 0.0057$ & $0.859 \pm 0.034$ & $0.493 \pm 0.020$ \\
800 & $0.6170 \pm 0.0057$ & $0.862 \pm 0.037$ & $0.532 \pm 0.023$ \\
850 & $0.6165 \pm 0.0057$ & $0.855 \pm 0.039$ & $0.527 \pm 0.025$ \\
900 & $0.6056 \pm 0.0059$ & $0.870 \pm 0.044$ & $0.526 \pm 0.027$ \\
1000 & $0.6434 \pm 0.0068$ & $0.854 \pm 0.068$ & $0.549 \pm 0.044$ \\
\hline
\end{tabular}

Table 7.6: Z' signal acceptance in CCCC topology. 


\begin{tabular}{|cccc|}
\hline $\begin{array}{c}\text { Mass } \\
(\mathrm{GeV})\end{array}$ & $\begin{array}{c}\text { Geometric } \\
\text { Acceptance }\end{array}$ & $\begin{array}{c}\text { Window } \\
\text { Efficiency }\end{array}$ & $\begin{array}{c}\text { Total } \\
\text { Acceptance }\end{array}$ \\
\hline \hline 400 & $0.1556 \pm 0.0039$ & $0.640 \pm 0.02$ & $0.0996 \pm 0.0048$ \\
500 & $0.1419 \pm 0.0038$ & $0.606 \pm 0.032$ & $0.0860 \pm 0.0044$ \\
600 & $0.1128 \pm 0.0036$ & $0.573 \pm 0.029$ & $0.0646 \pm 0.0036$ \\
650 & $0.0848 \pm 0.0031$ & $0.556 \pm 0.033$ & $0.0472 \pm 0.0029$ \\
700 & $0.0866 \pm 0.0031$ & $0.539 \pm 0.035$ & $0.0463 \pm 0.0027$ \\
750 & $0.0826 \pm 0.0031$ & $0.523 \pm 0.033$ & $0.0432 \pm 0.0029$ \\
800 & $0.0529 \pm 0.0026$ & $0.506 \pm 0.048$ & $0.0268 \pm 0.0023$ \\
850 & $0.0611 \pm 0.0028$ & $0.489 \pm 0.041$ & $0.0299 \pm 0.0025$ \\
900 & $0.0568 \pm 0.0028$ & $0.472 \pm 0.039$ & $0.0268 \pm 0.0021$ \\
1000 & $0.0310 \pm 0.0025$ & $0.439 \pm 0.063$ & $0.0136 \pm 0.0022$ \\
\hline
\end{tabular}

Table 7.7: Z' signal acceptance in ECCC topology.

\begin{tabular}{|cc|}
\hline $\begin{array}{c}\text { Mass } \\
(\mathrm{GeV})\end{array}$ & $\begin{array}{c}\text { Total } \\
\text { Acceptance }\end{array}$ \\
\hline \hline 400 & $0.521 \pm 0.018$ \\
500 & $0.524 \pm 0.018$ \\
600 & $0.535 \pm 0.019$ \\
650 & $0.531 \pm 0.020$ \\
700 & $0.535 \pm 0.020$ \\
750 & $0.538 \pm 0.021$ \\
800 & $0.561 \pm 0.024$ \\
850 & $0.558 \pm 0.025$ \\
900 & $0.548 \pm 0.028$ \\
1000 & $0.566 \pm 0.045$ \\
\hline
\end{tabular}

Table 7.8: Z' total signal acceptance for the total combined sample, CCCC and ECCC. 


\subsection{Theoretical Signal Production Cross Sections}

The LO signal production cross-section is determined from PYTHIA [73]. As mentioned previously, to account for NLO effects, a mass independent k-factor of 1.3 [15] is applied. Additionally the theoretical cross section for each mass point is multiplied by the $\sqrt{\hat{s}}$ acceptance. Table 7.9 under "Theoretical Cross Section" the resultant values including the uncertainties. In the limit figures, the theoretical cross section will be shown as a band.

\begin{tabular}{|ccccc|}
\hline $\begin{array}{c}\text { Mass } \\
(\mathrm{GeV})\end{array}$ & $\begin{array}{c}\text { Generated } \\
\text { Cross Section }(\mathrm{pb})\end{array}$ & $\begin{array}{c}\sqrt{\hat{s}} \\
\text { Acceptance }\end{array}$ & k-factor & $\begin{array}{c}\text { Theoretical } \\
\text { Cross Section }(\mathrm{pb})\end{array}$ \\
\hline \hline 400 & 1.118 & $0.9426 \pm 0.0024$ & 1.3 & $1.370 \pm 0.110$ \\
500 & 0.394 & $0.9282 \pm 0.0027$ & 1.3 & $0.476 \pm 0.038$ \\
600 & 0.146 & $0.8953 \pm 0.0033$ & 1.3 & $0.170 \pm 0.014$ \\
650 & 0.090 & $0.8796 \pm 0.0034$ & 1.3 & $0.1031 \pm 0.0083$ \\
700 & 0.055 & $0.8543 \pm 0.0036$ & 1.3 & $0.0622 \pm 0.0049$ \\
750 & 0.034 & $0.8435 \pm 0.0038$ & 1.3 & $0.0376 \pm 0.0030$ \\
800 & 0.021 & $0.7847 \pm 0.0043$ & 1.3 & $0.0216 \pm 0.0017$ \\
850 & 0.013 & $0.7528 \pm 0.0044$ & 1.3 & $0.0127 \pm 0.0010$ \\
900 & 0.008 & $0.6977 \pm 0.0046$ & 1.3 & $0.00735 \pm 0.00059$ \\
1000 & 0.003 & $0.5331 \pm 0.0052$ & 1.3 & $0.00219 \pm 0.00017$ \\
\hline
\end{tabular}

Table 7.9: Z' signal theoretical cross section.

\subsection{Limit Calculation}

The intersection of the graphs from the theoretical cross section band and the calculated upper cross section at 95\% CL gives the minimum allowed mass that a sequential Z' boson may have. For a conservative limit, the lower edge of the cross theoreti- 
cal cross section band is used. Observed and expected, mass limits are calculated for combined CCCC, ECCC sub-samples, as well as for the combined total sample. For the CCCC topology the combination is done by adding the contributions of the two sub-samples and re-calculating the limit. In order to combine both CCCC and ECCC, the luminosity weighted total acceptance luminosity ${ }_{C C C C} \times$ acceptance $_{C C C C}+$ luminosity $_{E C C C} \times$ acceptance $_{E C C C}$ is used.

For the combined CCCC topology, an expected limit of $924 \mathrm{GeV}$ and an observed limit of $917 \mathrm{GeV}$ are obtained (Figure 7.2, upper plot).

For the ECCC topology, expected and observed lower mass limits of $695 \mathrm{GeV}$ are obtained (Figure 7.2, lower plot).

For the combination of the CCCC and ECCC topologies, an expected limit of $927 \mathrm{GeV}$ and an observed limit of $920 \mathrm{GeV}$ are obtained (Figure 7.3). 

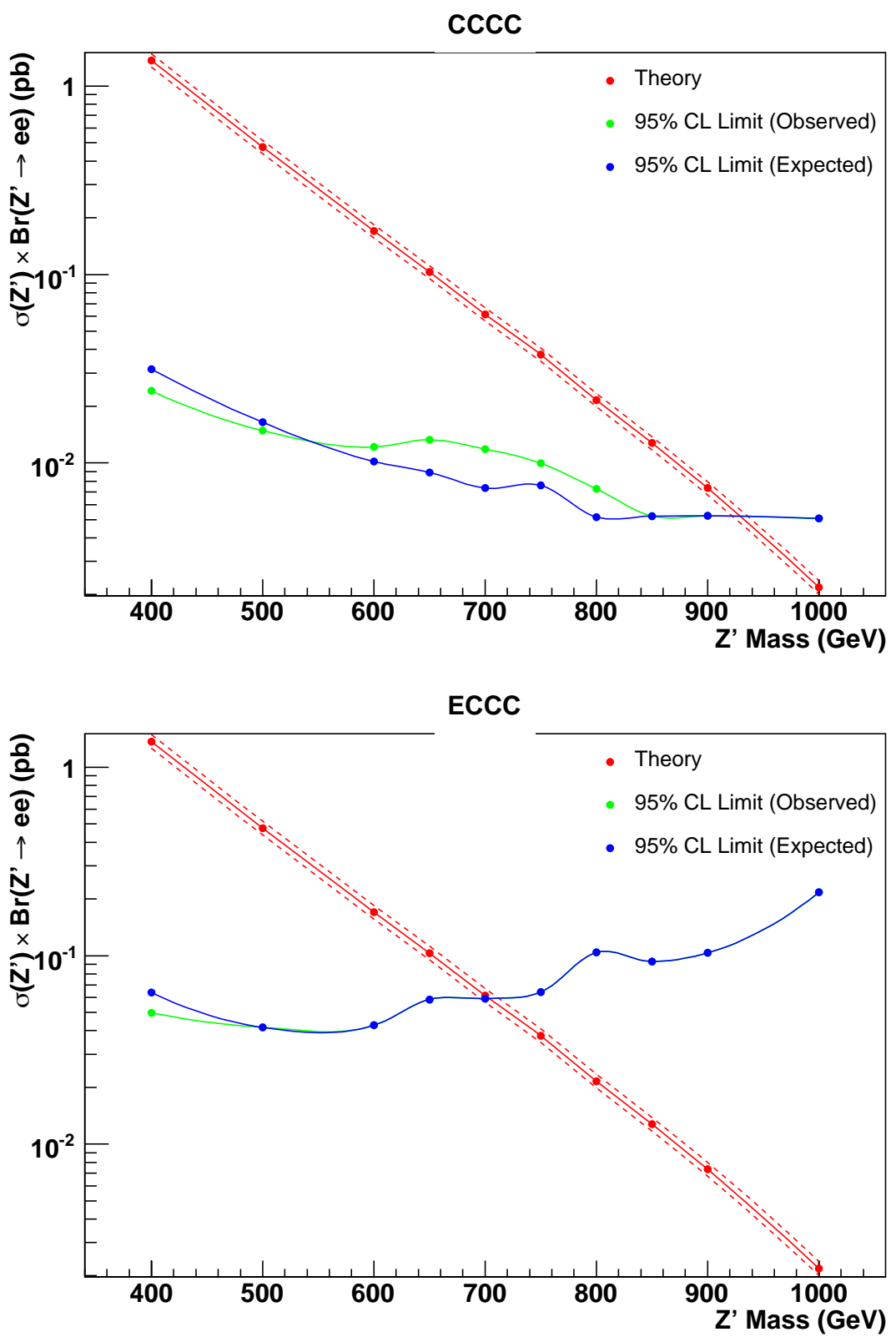

Figure 7.2: 95\% CL limit on $\sigma \times B R\left(\mathrm{Z}^{\prime} \rightarrow e^{+} e^{-}\right)$for CCCC, and ECCC. Upper plot corresponds to the CCCC, and the bottom plot to ECCC. 


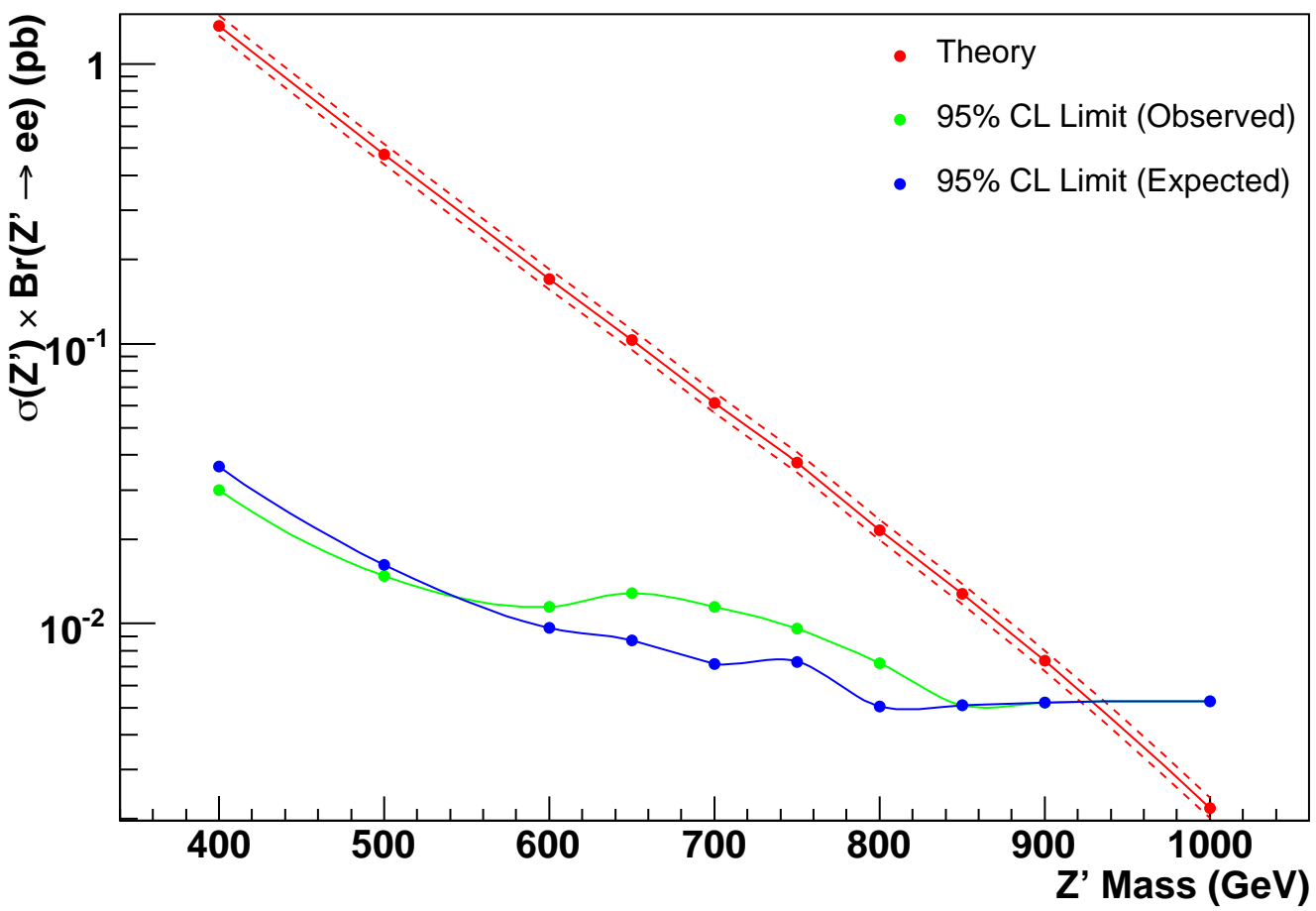

Figure 7.3: 95\% CL limit on $\sigma \times B R\left(\mathrm{Z}^{\prime} \rightarrow e^{+} e^{-}\right)$for the total combined sample, CCCC and ECCC. 


\section{Chapter 8}

\section{Summary}

\subsection{Summary}

A search for evidence of $p \bar{p} \rightarrow \mathrm{Z}^{\prime} \rightarrow e^{+} e^{-}$has been performed using data collected with the D0 detector in the Tevatron $p \bar{p}$ collisions at $\sqrt{s}=1.96 \mathrm{TeV}$ from 2002 to 2006. The observed number of data events is consistent with the SM predictions, and no evidence of a $Z^{\prime}$ signal is observed. The existence of a $Z^{\prime}$ with a mass less than $920 \mathrm{GeV}$ at the $95 \%$ confidence level is excluded, assuming a $\mathrm{Z}^{\prime}$ with the same couplings as the Standard Model $Z$. This result significantly improves the previous D0 result of $719 \mathrm{GeV}$ using $122 \mathrm{pb}^{-1}$ of data [22], and the previous published CDF result of $850 \mathrm{GeV}[23]$.

The Tevatron is expected to continue its operation until at least the end of 2009 with an integrated luminosity of more than $6 \mathrm{fb}^{-1}$ expected to be delivered. Assuming that no signal is found, Tevatron will be able to probe for $\mathrm{Z}^{\prime}$ bosons with masses up to $\sim 1 \mathrm{TeV}$. Above that is the range that LHC is expected to explore.

The LHC, expected to begin operations by the end of 2008 , is a $p p$ collider with 
$\sqrt{s}=14 \mathrm{TeV}$. The LHC is expected to collect about $100 \mathrm{fb}^{-1}$, and will be able to search for $\mathrm{Z}^{\prime}$ bosons with masses up to $\sim 5 \mathrm{TeV}$ [18]. Upgrade plans for the LHC would even further increase the mass reach. 


\section{Bibliography}

[1] The LEP collaborations: A combination of preliminary electroweak measurements and constraints on the standard model (2005). arxiv:hep-ex/0511027v2.

[2] Leader, E. \& Predazzi, E. An Introduction to Gauge Theories and Modern Particle Physics, Volume 1: Electroweak interactions, the "new" particles and the parton model., vol. 3 (Cambridge University Press, 1996).

[3] Halzen, F. \& Martin, A. D. Quarks and Leptons: An Introductory Course in Modern Particle Physics (New York, USA: Wiley, 1984).

[4] Pich, A. The Standard Model of electroweak interactions. In Proceedings of the 2006 European School of High-Energy Physics, 1-51 (Aronsborg, Sweden, 2007).

[5] Griffiths, D. J. Introduction to Elementary Particles (New York, USA: Wiley, 1987).

[6] Quigg, C. Gauge Theories of the Strong, Weak and Electromagnetic Interactions, vol. 56 (Westview Press, 1983).

[7] Yao, W. M. et al. Review of particle physics. J. Phys. G33, 1-1232 (2006). URL http: //pdg. Ibl.gov. 
[8] Fukuda, Y. et al. Evidence for oscillation of atmospheric neutrinos. Phys. Rev. Lett. 81, 1562-1567 (1998).

[9] Ahmad, Q. R. et al. Direct evidence for neutrino flavor transformation from neutral-current interactions in the Sudbury Neutrino Observatory. Phys. Rev. Lett. 89, 011301 (2002).

[10] Arnold, R. et al. First results of the search of neutrinoless double beta decay with the NEMO 3 detector. Phys. Rev. Lett. 95, 182302 (2005).

[11] Gabrielse, G. et al. New determination of the fine structure constant from the electron g value and QED. Phys. Rev. Lett. 97 (2006).

[12] Glashow, S. L. Partial symmetries of weak interactions. Nucl. Phys. 22, 579-588 (1961).

[13] Pumplin, J. et al. New generation of parton distributions with uncertainties from global QCD analysis. JHEP 0207, 012 (2002).

[14] Barate, R. et al. Search for the standard model higgs boson at LEP. Phys. Lett. B565, 61-75 (2003).

[15] Carena, M. et al. Z' gauge bosons at the Tevatron (2004). Arxiv:hep$\mathrm{ph} / 0408098 \mathrm{v} 1$.

[16] Cvetic, M. \& Godfrey, S. Discovery and identification of extra gauge bosons (1995). Hep-ph/9504216.

[17] Langacker, P. The physics of heavy Z' gauge bosons (2008). ArXiv:0801.1345v2 [hep-ph].

[18] Rizzo, T. Z' phenomenology and the LHC (2006). ArXiv:hep-ph/0610104v1. 
[19] Leike, A. The phenomenology of Extra Neutral Gauge Bosons (1998). ArXiv:hep$\mathrm{ph} / 9805494 \mathrm{v} 1$.

[20] Mohapatra, R. N. Unification and Supersymmetry (New York, USA:Springer Verlag, 1992).

[21] Kaku, M. Quantum Field Theory: A Modern Introduction (New York, USA:Oxford University Press, 1993).

[22] Gao, M. A search for Extra Neutral Gauge Boson in the Dielectron Channel. Ph.D. thesis, Columbia University (2003).

[23] Abulencia, A. et al. Search for $\mathrm{Z}^{\prime} \rightarrow e^{+} e^{-}$using dielectron mass and angular distribution. Phys. Rev. Lett. 96, 211801 (2006).

[24] Thompson, J. Introduction to colliding beams at Fermilab (1994). FERMILABTM-1909.

[25] Design report Tevatron 1 project (1984). FERMILAB-DESIGN-1984-01.

[26] Run II handbook. URL http://www-bd.fnal.gov/lug/runII_handbook/RunII_index.html.

[27] Schmidt, C. W. \& Curtis, C. D. A 50-milliampere negative hydrogen ion source. IEEE Trans. Nucl. Sci. 26, 4120-4122 (1979).

[28] Patterson, D. The FNAL 200-MeV Linac (1986). FERMILAB-MISC-1986-01.

[29] Provided by Fermilab Visual Media Services.

[30] Curtis, C. D. et al. Linac $H^{-}$beam operation and uses at Fermilab. IEEE Trans. Nucl. Sci. 26, 3760-3762 (1979). 
[31] Fermilab Linac upgrade conceptual design revision 4A (1989). FERMILAB-LUCONCEPTUAL-DESIGN.

[32] Hubbard, E. L. Booster synchrotron (1973). FERMILAB-TM-0405.

[33] Hojvat, C. et al. The multiturn charge exchange injection system for the Fermilab Booster accelerator. IEEE Trans. Nucl. Sci. 26, 3149-3151 (1979).

[34] The Fermilab Main Injector technical design handbook. URL http://www-fmi.fnal.gov/fmiinternal/MI_Technical_Design/index.html.

[35] The antiproton source rookie book, version 1.1 (1999). URL http://www-bdnew.fnal.gov/pbar/documents/PBAR_Rookie_Book.pdf.

[36] Jackson, G. The Fermilab recycler ring technical design report. rev. 1.2 (1996). FERMILAB-TM-1991.

[37] Nagaitsev, S. et al. Experimental demonstration of relativistic electron cooling. Phys. Rev. Lett. 96, 044801 (2006).

[38] Abachi, S. et al. The D0 detector. Nucl. Instrum. Meth. A338, 185-253 (1994).

[39] Abazov, V. M. et al. The upgraded D0 detector. Nucl. Instrum. Meth. A565, 463-537 (2006).

[40] D0 Upgrade Collaboration. D0 silicon tracker technical design report (1994). URL http://www-d0.fnal.gov/ Iipton/tdr_final.ps. D0 Note 2169.

[41] Adams, D. et al. The D0 upgrade: Central fiber tracker, technical design report (1999). D0 Note 4164.

[42] Brzezniak, J. et al. Conceptual design of a 2 Tesla superconducting solenoid for the Fermilab D0 detector upgrade (1994). FERMILAB-TM-1886. 
[43] D0 Upgrade Collaboration. Design report of the central preshower for the D0 detector (1996). D0 Note 3104.

[44] Katsanos, I. et al. Forward preshower system calibration and energy saturation study (2006). D0 Note 5283.

[45] Patwa, A. The Forward Preshower System and a Study of the J/ $\psi$ Trigger with the DØ Detector. Ph.D. thesis, SUNY, Stony Brook (2002).

[46] Leo, W. R. Techniques for Nuclear and Particle Physics Experiments: A How-to Approach (Berlin, Germany: Springer, 1987).

[47] Fabjan, C. W. \& Gianotti, F. Calorimetry for particle physics. Rev. Mod. Phys. 75, 1243-1286 (2003).

[48] Edwards, T. L. et al. Determination of the effective inelastic $p \bar{p}$ cross-section for the D0 Run II luminosity measurement. FERMILAB-TM-2278-E.

[49] Andeen, T. et al. The D0 experiment's integrated luminosity for Tevatron Run IIa. FERMILAB-TM-2365.

[50] Adams, M. et al. Level-2 calorimeter preprocessor technical design report (1999). D0 Note 3615.

[51] D0 Run II software algorithms. URL http://www-d0.fnal .gov/computing/algorithms/.

[52] Kowalkowski, J. et al. D0 offline reconstruction and analysis control framework. In Proceedings of the International Conference on Computing in High-Energy Physics and Nuclear Physics (CHEP 2000) (Padova, Italy, 2000).

[53] Khanov, A. HTF: histogramming method for finding tracks. the algorithm descpription. (2000). D0 Note 3778. 
[54] Borissov, G. Ordering a chaos or... techical details of AA tracking (2003). URL http://www-d0.fnal.gov/atwork/adm/d0_private/2003-02-28/adm_talk.ps.

[55] Frühwirth, R. Application of Kalman filtering to track and vertex fitting. Nucl. Instrum. Meth. A262, 444-450 (1987).

[56] Greenlee, H. The D0 Kalman track fit (2004). D0 Note 4303.

[57] Schwartzman, A. \& Tully, C. Primary vertex reconstruction by means of adaptive vertex fitting (2005). D0 Note 4918.

[58] Schwartzman, A. \& Narain, M. Probabilistic primary vertex selection (2002). URL http://www-clued0.fnal.gov/ ${ }^{\sim}$ aran/m_4042.ps. D0 Note 4042.

[59] Peters, Y. et al. Certification of the adaptive primary vertex in p17 (2006). D0 Note 5192.

[60] Bernardi, G. et al. NADA: a new event by event hot cell killer (2000). D0 Note 3687.

[61] Bernardi, G. \& Trincaz-Duvoid, S. Improvement of the NADA algorithm: Hot cell killing in D0 Run II data (2002). D0 Note 4057.

[62] Bernardi, G. \& Kado, M. Hot cell suppression at level 3 (2003). D0 Note 4039.

[63] Bassler, U. \& Bernardi, G. Towards a coherent treatment of calorimetric energies: Missing transverse energy, jets, E.M. objects and the T42 algorithm (2002). D0 Note 4124.

[64] Vlimant, J.-R. et al. Technical description of the T42 algorithm for the calorimeter noise suppression (2003). D0 Note 4146. 
[65] Bernardi, G. et al. Improvements from the T42 algorithm on calorimeter objects reconstruction (2004). D0 Note 4335.

[66] Hadley, N. Cone algorithm for jet finding (1989). D0 Note 904.

[67] Kozminski, J. et al. Electron likelihood in p14 (2004). D0 Note 4449.

[68] Getting started with electron/photon ID. URL http://www-d0.fnal.gov/phys_id/emid/d0_private/emid_intro.html.

[69] Kumar, A. et al. Electron likelihood study (2005). D0 Note 4769.

[70] Hays, J. et al. Single electron efficiencies in p17 data and Monte-Carlo using p18.05.00 d0correct (2006). D0 Note 5105.

[71] Narain, M. Electron identification in the D0 detector. In The Fermilab Meeting: DPF 92 (Batavia, 1992). FERMILAB-CONF-93-054-E.

[72] Private communication with Jovan Mitrevski.

[73] Sjöstrand, T. et al. PYTHIA 6.4 physics and manual. JHEP 05, 026 (2006).

[74] Brun, R. \& Carminati, F. GEANT detector description and simulation tool (1993). CERN Program Library Long Writeup W5013.

[75] Hamberg, R. et al. A complete calculation of the order alpha- $s^{2}$ correction to the Drell-Yan k-factor. Nucl. Phys B 359 (1991). [Erratum-ibid, B 644, 403 (2002)].

[76] Mangano, M. et al. ALPGEN, a generator for hard multiparton processes in hadronic collisions. JHEP 0307, 001 (2003). 
[77] Vorwerk, V. et al. Search for excited electrons in the eer channel with the Run IIa data set (2007). D0 Note 5505.

[78] Private communication with Jan Stark.

[79] The ROOT system home page. URL http://root.cern.ch/.

[80] Chapin, D. et al. Measurement of the cross section for $\mathrm{W}$ and $\mathrm{Z}$ production to electron final states with the D0 detector at $\sqrt{s}=1.96 \mathrm{TeV}$ (2004). D0 Note 4403.

[81] Private communication with Gustaaf Brooijmans.

[82] Bertram, I. et al. A recipe for the construction of confidence limits (2000). FERMILAB-TM-2104.

[83] Buescher, V. et al. Recommendation of the ad-hoc committee on limit-setting procedures to be used by D0 in Run II (2004). D0 Note 4629.

[84] Hogg, R. V. \& Tanis, E. A. Probability and Statistical Inference (Macmillan Publishing Company, 1988).

[85] Simple limit calculator. URL http://www-d0.fnal.gov/Run2Physics/limit_calc/limit_calc.html.

[86] Magass, C. Search for new Heavy Charged Gauge Bosons. Ph.D. thesis, RWTH Aachen University (2007).

[87] Nunnemann, T. NNLO cross sections for Drell-Yan, Z and W production using modern parton distribution functions (2004). URL http://www-clued0.fnal.gov/^nunne/cross-sections/dy_cross-sections . html. D0 Note 4476. 
[88] D0 pdf reweighting prcessor. URL http://www-d0.fnal.gov/DOCode/source/caf_pdfreweight/doc/index.html.

[89] Stump, D. et al. New generation of parton distributions with uncertainties from global QCD analysis. JHEP 0310, 046 (2003).

[90] D0 luminosity tools documentation. URL http://www-d0.fnal.gov/d0dist/dist/packages/lm_tools/devel/doc/. 
Appendix A

Figures for sub-samples 


\section{BACKGROUND NORMALIZATION PLOTS FOR "BOTH ELECTRONS" IN FIDUCIAL IN CCCC}
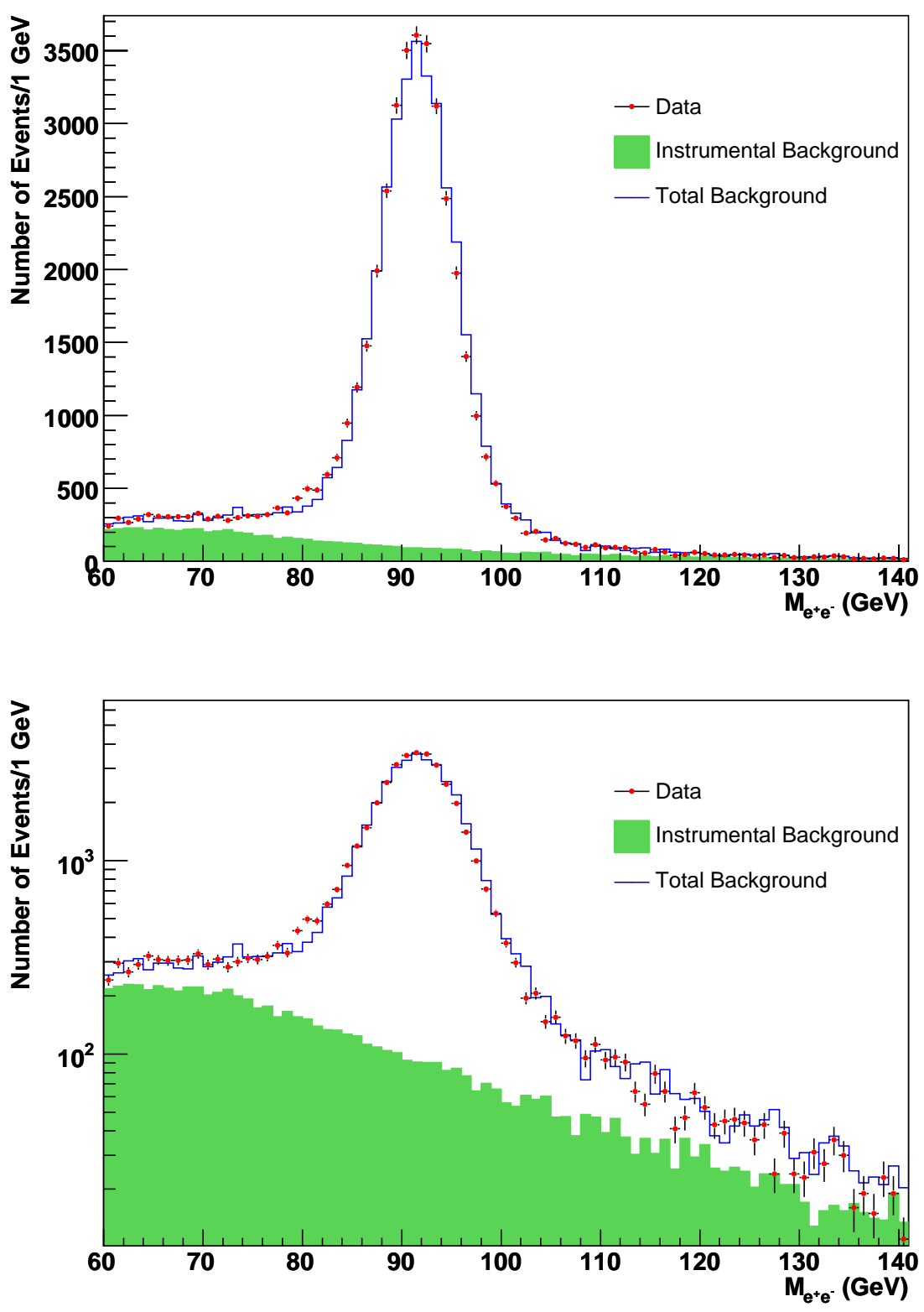

Figure A.1: Data/Background comparison of Invariant Mass distribution for both electron in fiducial in Central Calorimeter around $Z$ peak. The upper plot is in linear scale, and the bottom is in logarithmic. 


\section{BACKGROUND NORMALIZATION PLOTS FOR "ONE ELECTRON" IN FIDUCIAL IN CCCC}
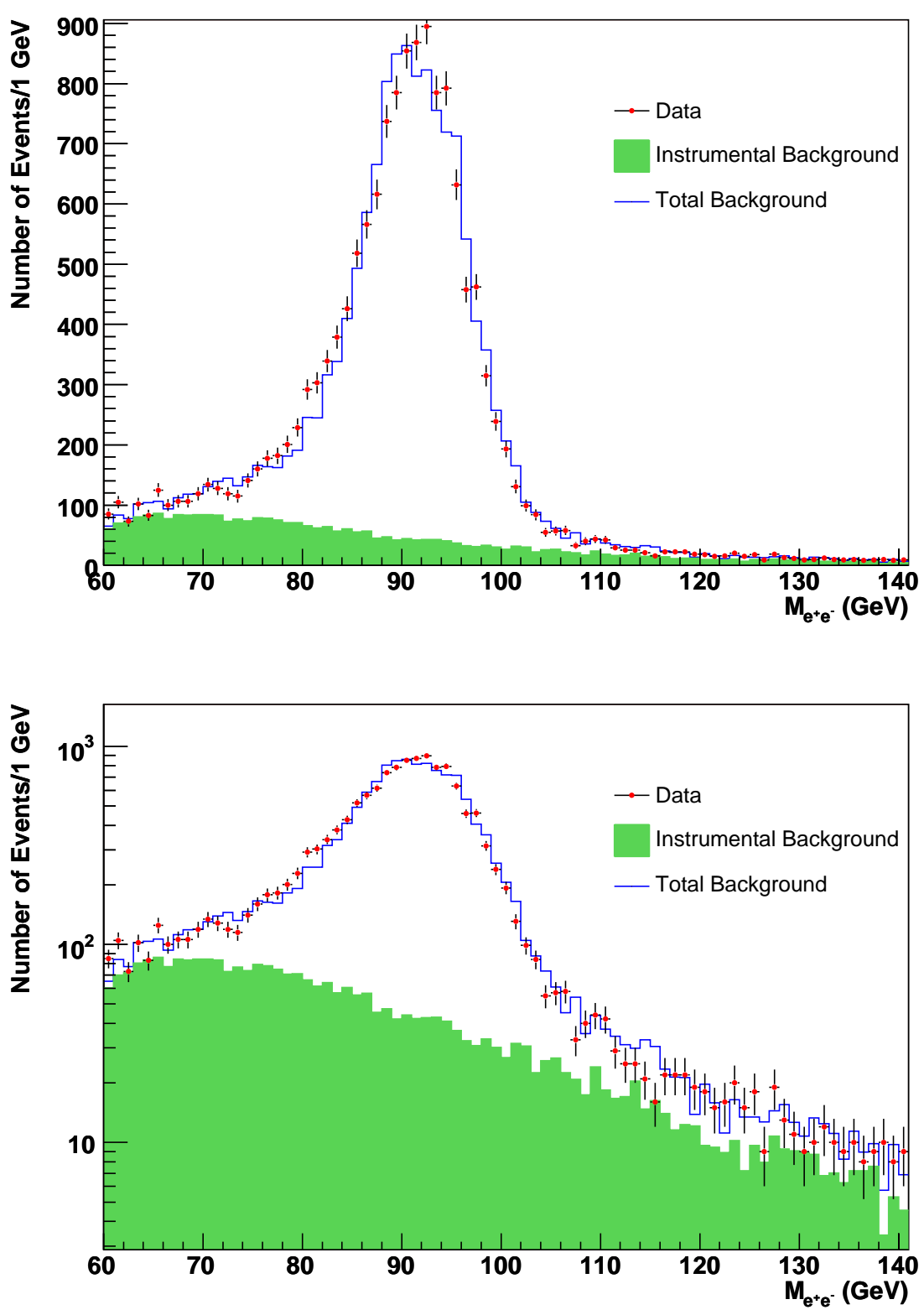

Figure A.2: Data/Background comparison of Invariant Mass distribution for just one electron in fiducial in Central Calorimeter around $Z$ peak. The upper plot is in linear scale, and the bottom is in logarithmic. 


\section{BACKGROUND NORMALIZATION PLOTS FOR "IN FIDUCIAL" IN ECCC}
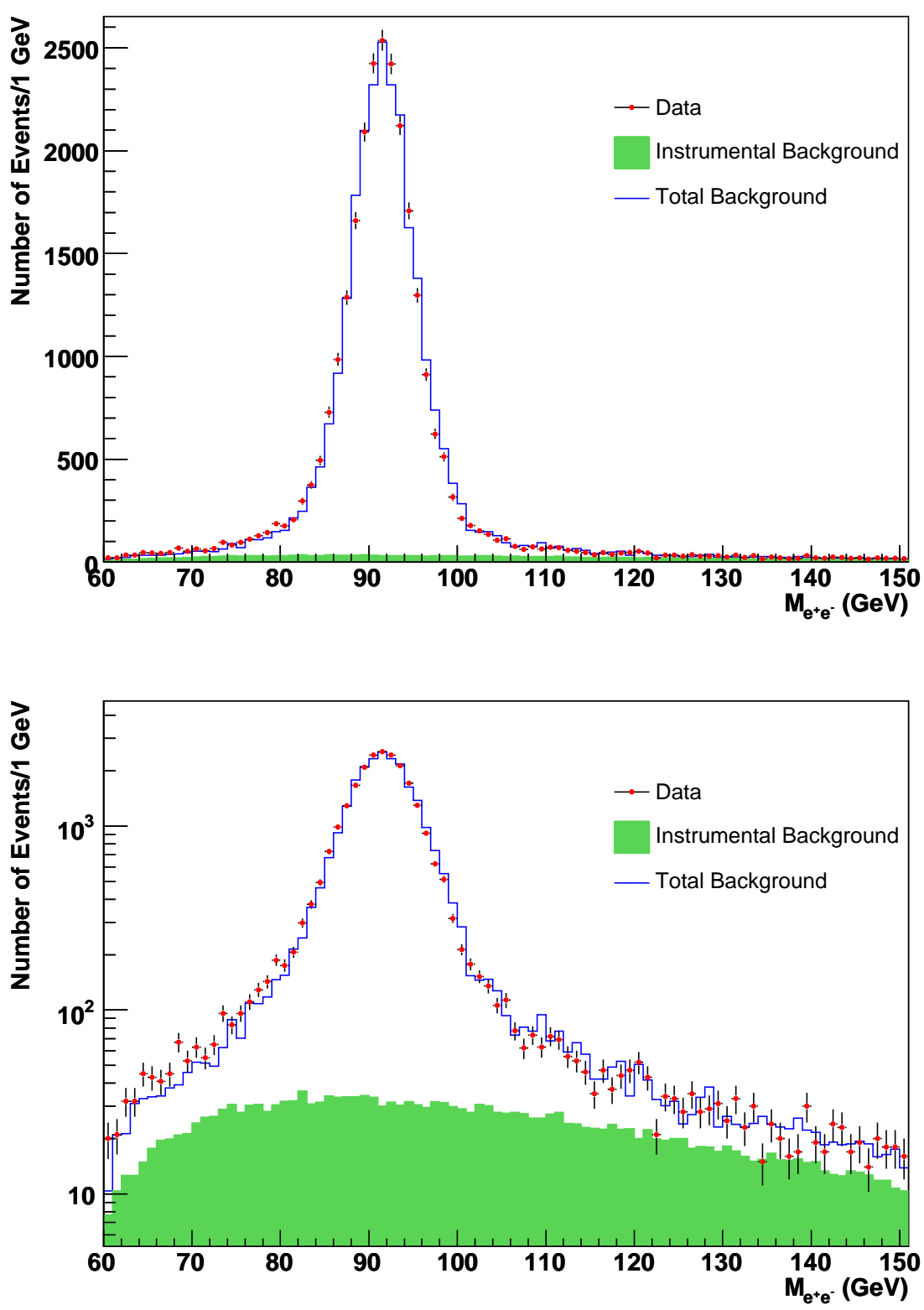

Figure A.3: Data/Background comparison of Invariant Mass distribution in ECCC around $Z$ peak, for the "in fiducial" sub-set. The upper plot is in linear scale, and the bottom is in logarithmic. 
EXTRAPOLATION TO HIGHER MASSES PLOT FOR "BOTH ELECTRONS" IN FIDUCIAL AND FOR "ONE ELECTRON" IN FIDUCIAL IN CCCC
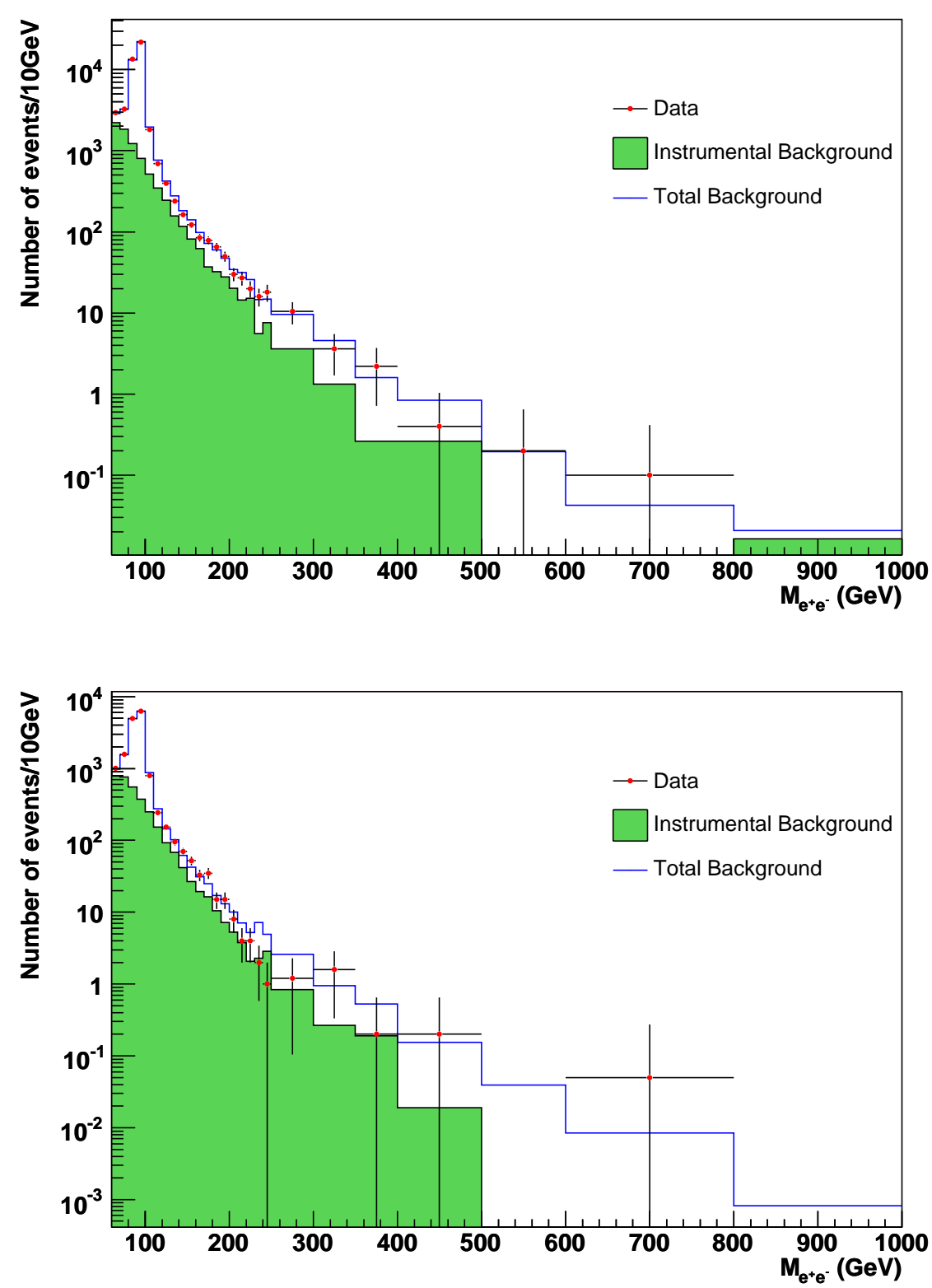

Figure A.4: Data/Background comparison of the full range Invariant Mass distribution for "both in fiducial" (upper plot) and "one in fiducial" (bottom plot) CCCC subsamples. 
EXTRAPOLATION TO HIGHER MASSES PLOT FOR "IN FIDUCIAL" IN ECCC

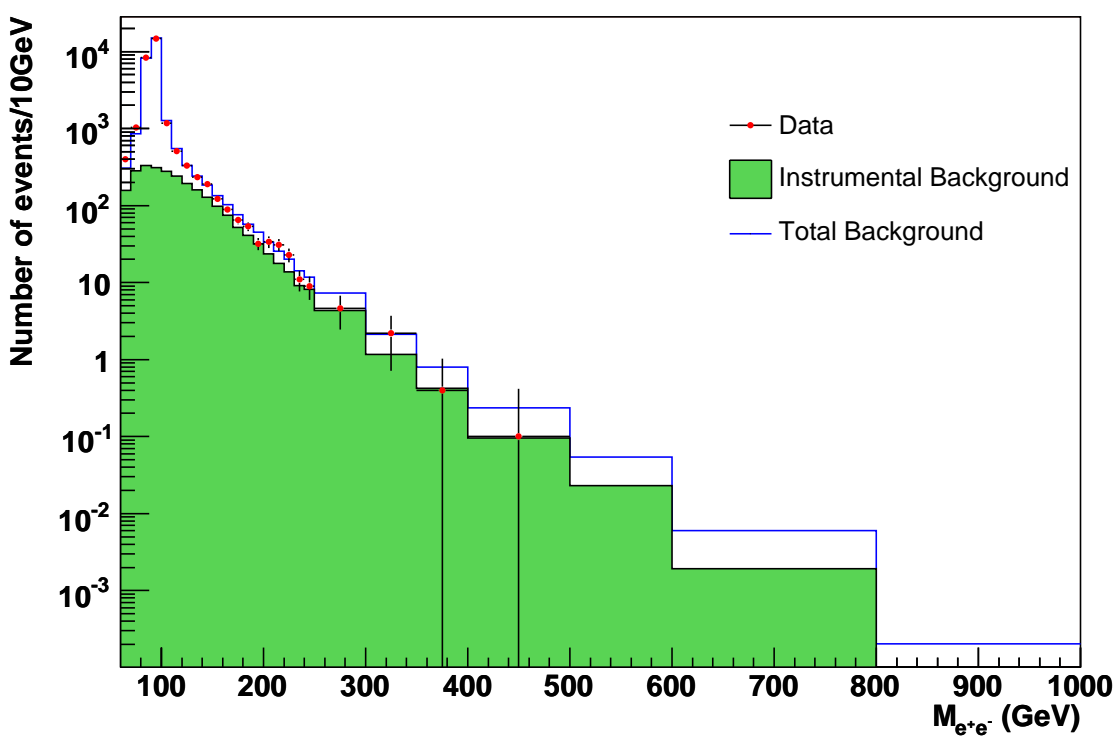

Figure A.5: Data/Background comparison of the full range Invariant Mass distribution for for "in fiducial" ECCC subsample. 
Appendix B

Z' Signal Figures 
FIGURES FOR A Z' $400 \mathrm{GeV}$ SIGNAL

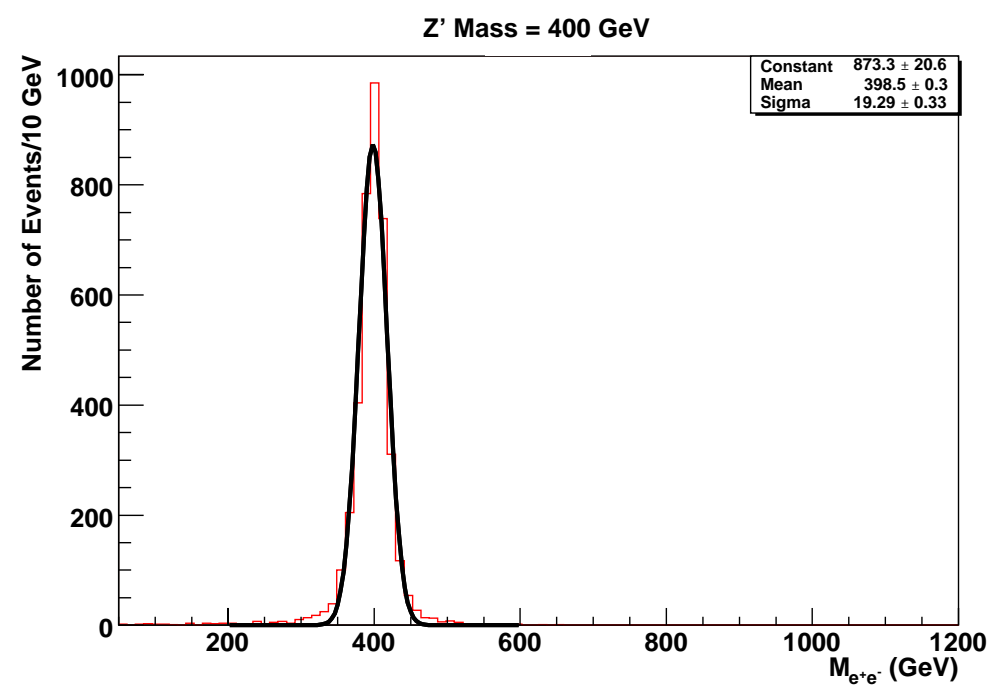

Figure B.1: Reconstructed MC invariant mass distribution for a potential Z' $400 \mathrm{GeV}$ signal fitted with a Gaussian.

\section{FIGURES FOR A Z' $500 \mathrm{GeV}$ SIGNAL}

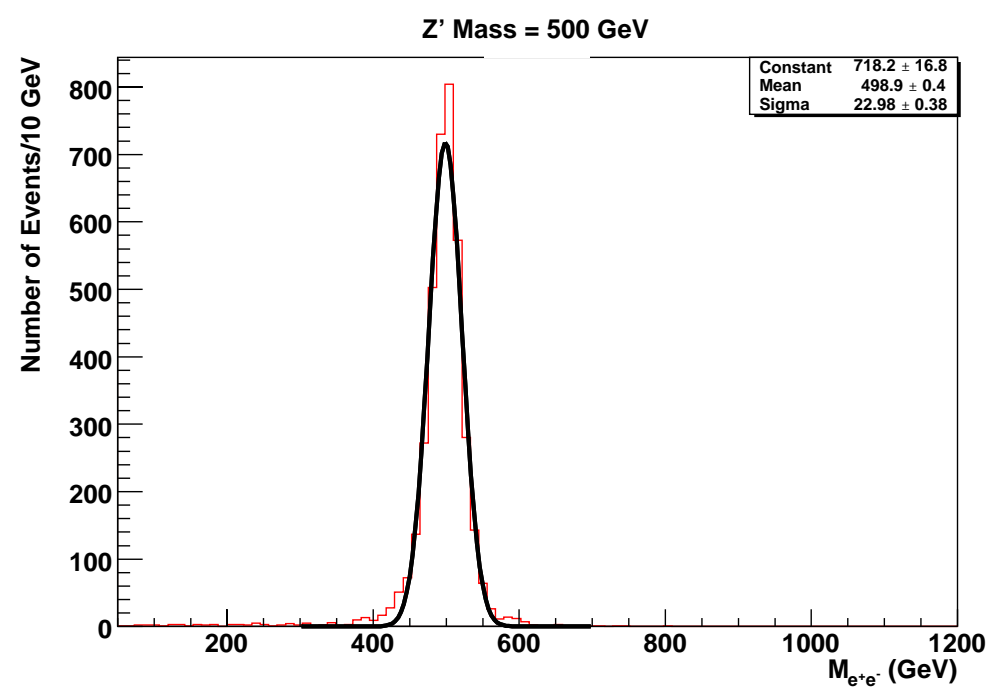

Figure B.2: Reconstructed MC invariant mass distribution for a potential Z' $500 \mathrm{GeV}$ signal fitted with a Gaussian. 
FIGURES FOR A Z' $600 \mathrm{GeV}$ SIGNAL

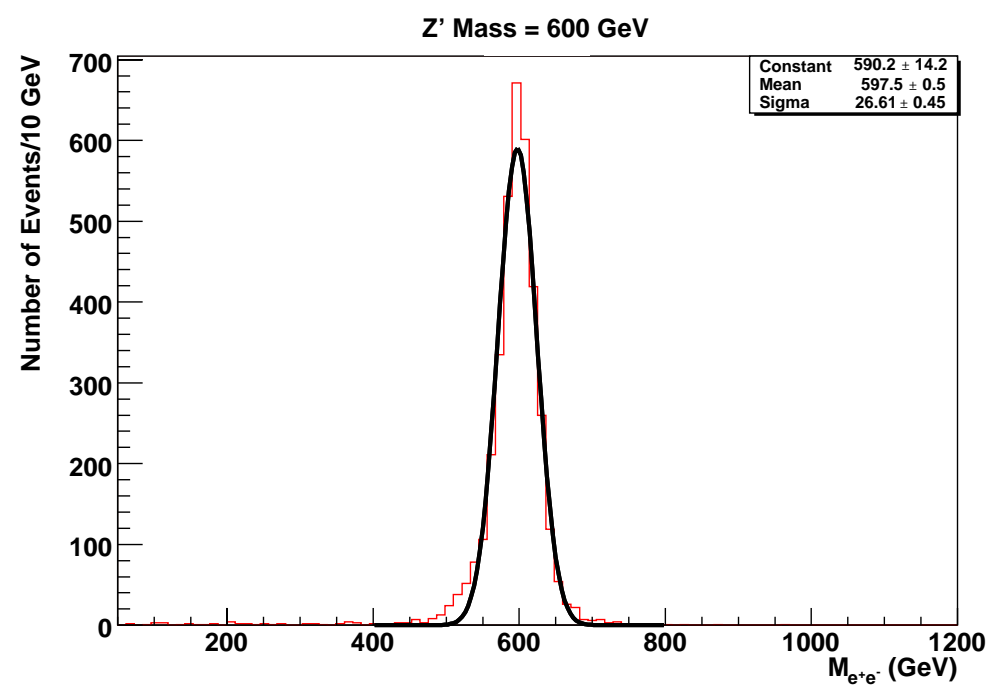

Figure B.3: Reconstructed MC invariant mass distribution for a potential Z' $600 \mathrm{GeV}$ signal fitted with a Gaussian.

FIGURES FOR A Z' $650 \mathrm{GeV}$ SIGNAL

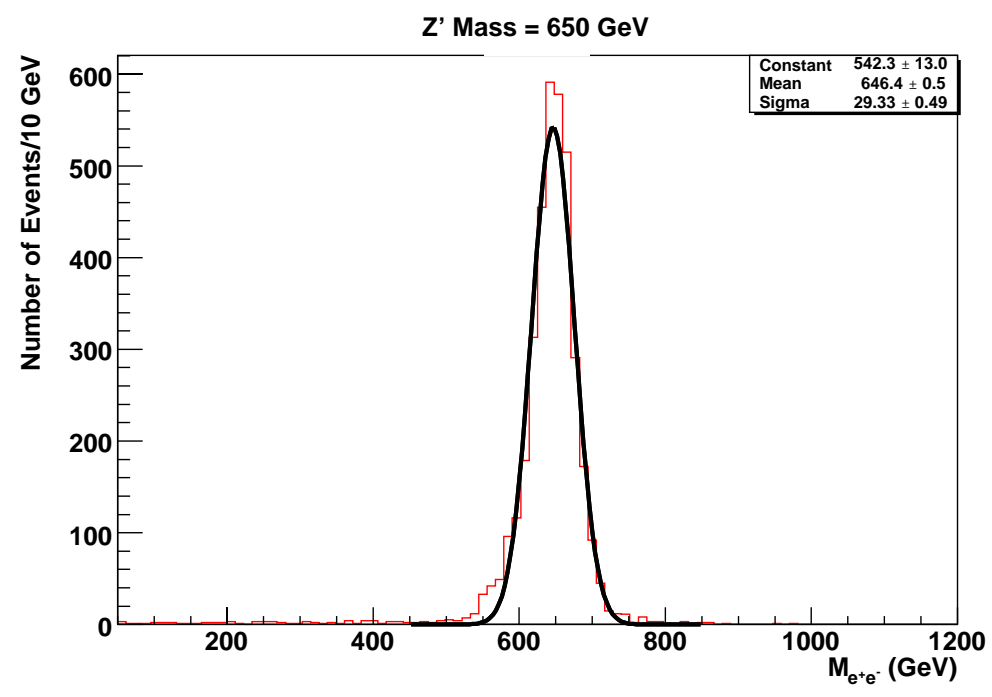

Figure B.4: Reconstructed MC invariant mass distribution for a potential Z' $650 \mathrm{GeV}$ signal fitted with a Gaussian. 
FIGURES FOR A Z' $700 \mathrm{GeV}$ SIGNAL

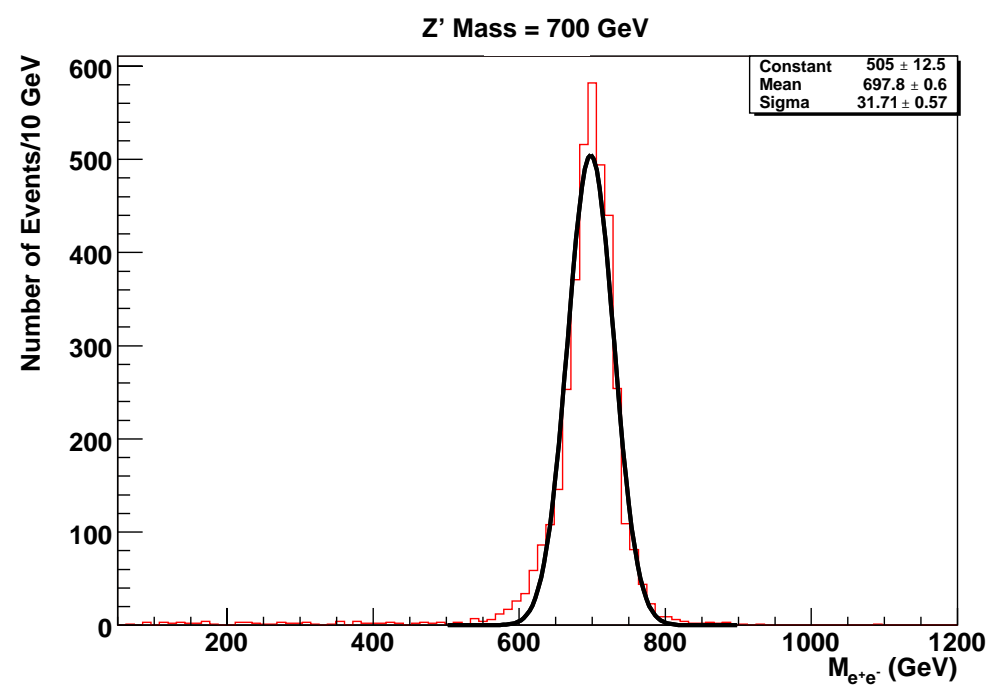

Figure B.5: Reconstructed MC invariant mass distribution for a potential $\mathrm{Z}^{\prime}$ $700 \mathrm{GeV}$ signal fitted with a Gaussian.

FIGURES FOR A Z' $750 \mathrm{GeV}$ SIGNAL

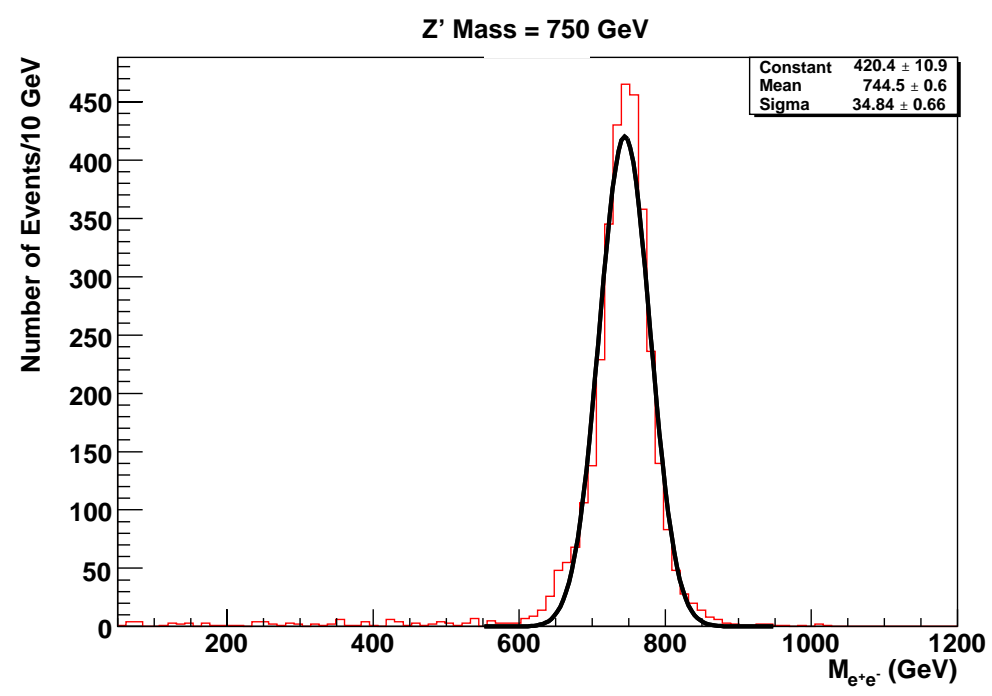

Figure B.6: Reconstructed MC invariant mass distribution for a potential Z' $750 \mathrm{GeV}$ signal fitted with a Gaussian. 


\section{FIGURES FOR A Z' $800 \mathrm{GeV}$ SIGNAL}

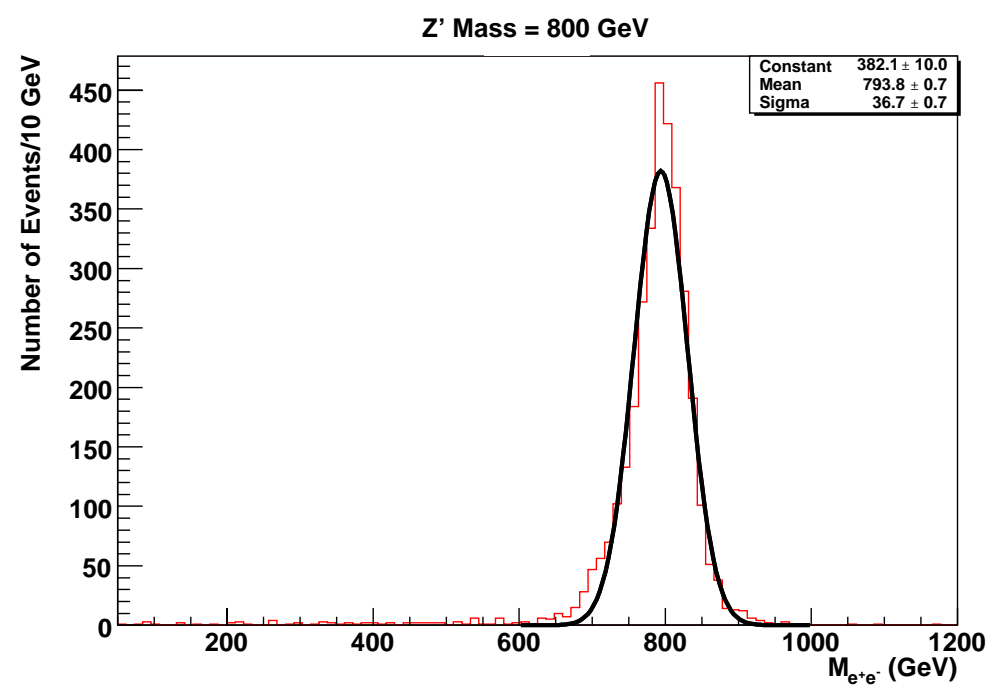

Figure B.7: Reconstructed MC invariant mass distribution for a potential Z' $800 \mathrm{GeV}$ signal fitted with a Gaussian.

FIGURES FOR A Z' $850 \mathrm{GeV}$ SIGNAL

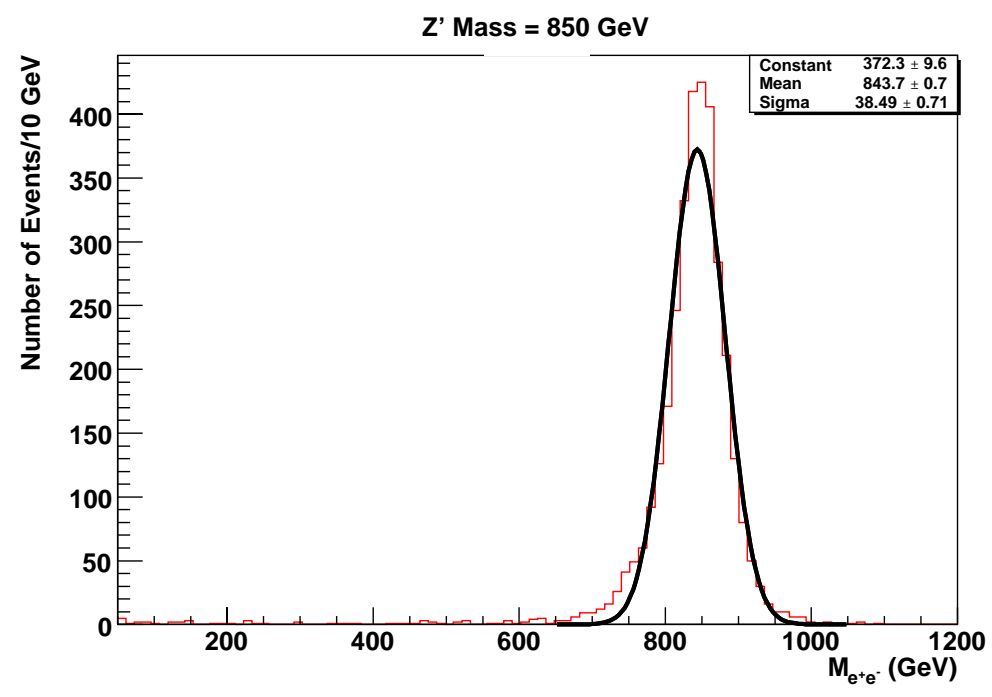

Figure B.8: Reconstructed MC invariant mass distribution for a potential Z' $850 \mathrm{GeV}$ signal fitted with a Gaussian. 
FIGURES FOR A Z' $900 \mathrm{GeV}$ SIGNAL

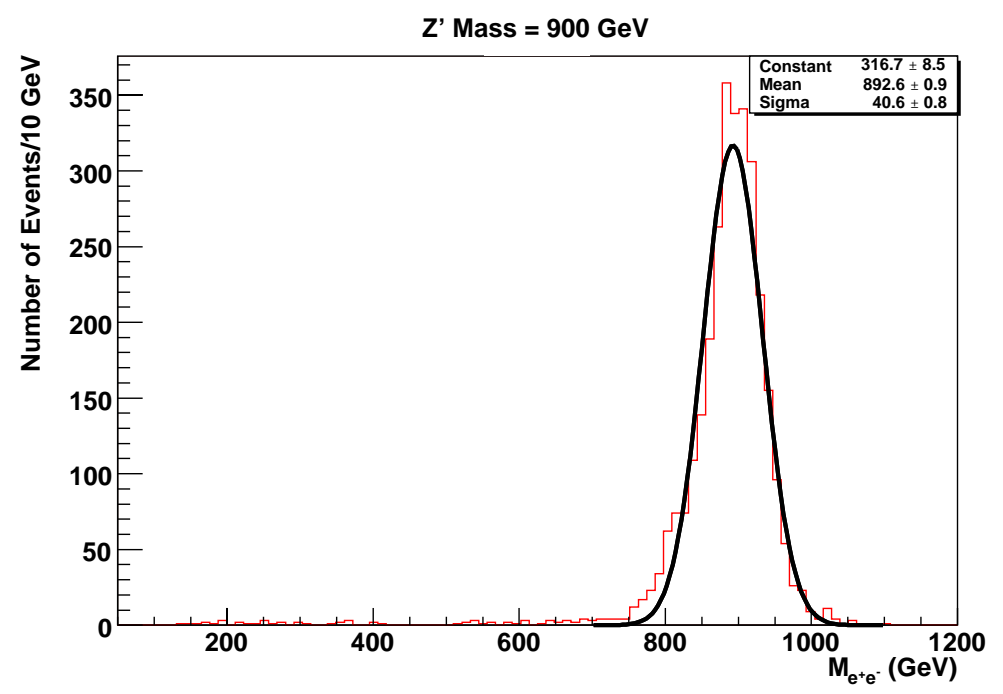

Figure B.9: Reconstructed MC invariant mass distribution for a potential Z' $900 \mathrm{GeV}$ signal fitted with a Gaussian.

FIGURES FOR A Z' $1000 \mathrm{GeV}$ SIGNAL

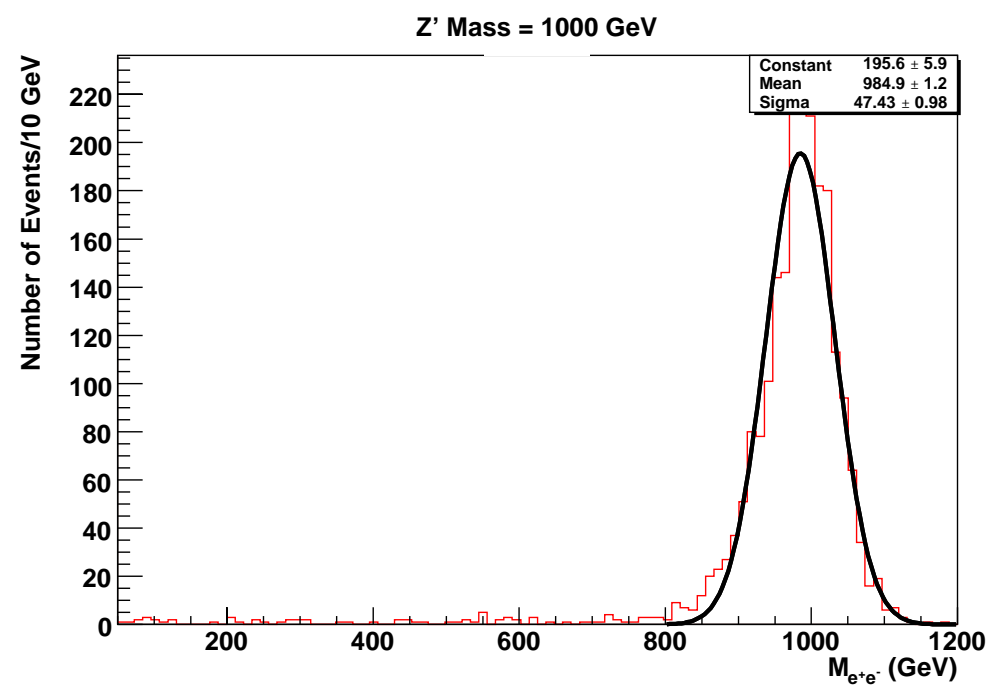

Figure B.10: Reconstructed MC invariant mass distribution for a potential Z' $1000 \mathrm{GeV}$ signal fitted with a Gaussian. 
Appendix $\mathrm{C}$

\section{Tables for sub-samples}




\begin{tabular}{|cccc|c|}
\hline $\begin{array}{c}\text { Mass } \\
(\mathrm{GeV})\end{array}$ & $\begin{array}{c}\text { Data } \\
\text { Events }\end{array}$ & \multicolumn{2}{c|}{ Expected Background } \\
Events
\end{tabular}

Table C.1: Number of events above mass for both electrons in fiducial subsample. 


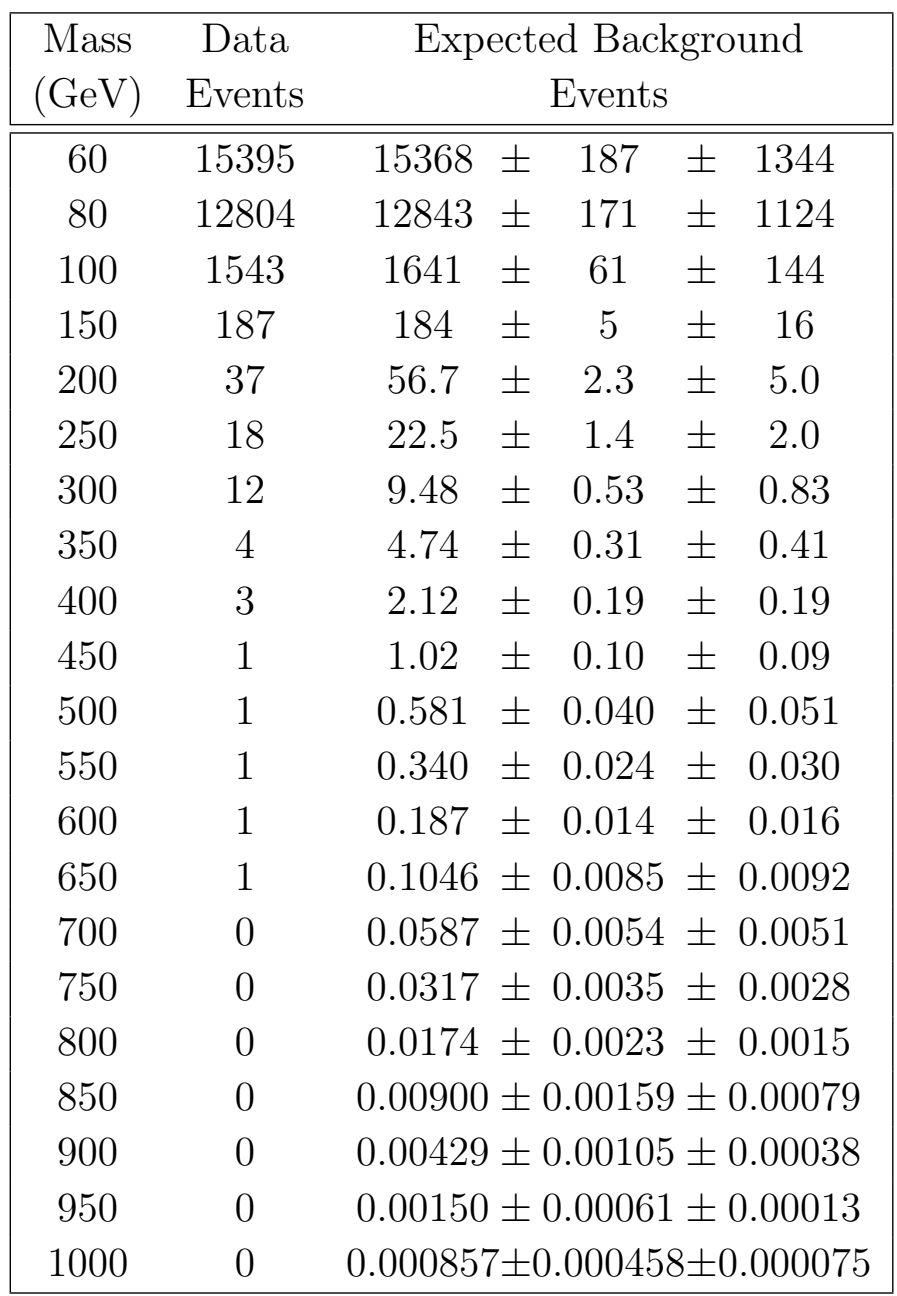

Table C.2: Number of events above mass for just one electron in fiducial sub-sample. 


\begin{tabular}{|c|c|c|}
\hline $\begin{array}{l}\text { Mass } \\
(\mathrm{GeV})\end{array}$ & $\begin{array}{c}\text { Data } \\
\text { Events }\end{array}$ & $\begin{array}{c}\text { Expected Background } \\
\text { Events }\end{array}$ \\
\hline 60 & 27539 & $27473 \pm 734 \quad \pm 2404$ \\
\hline 80 & 26112 & $674 \pm 2302$ \\
\hline 100 & 2942 & $3140 \pm$ \\
\hline 150 & 508 & $570 \pm$ \\
\hline 200 & 145 & $158 \pm$ \\
\hline 250 & 37 & $53.88 \pm$ \\
\hline 300 & 14 & $17.5 \pm$ \\
\hline 350 & 3 & $7.0 \pm 0.71 \pm 0.61$ \\
\hline 400 & 1 & $3.01 \pm 0.30 \pm 0.26$ \\
\hline 450 & 1 & $1.18 \pm 0.13 \pm 0.10$ \\
\hline 500 & 0 & $0.660 \pm 0.074 \pm 0.058$ \\
\hline 550 & 0 & $0.298 \pm 0.029 \pm 0.026$ \\
\hline 600 & 0 & $0.124 \pm 0.012 \pm 0.011$ \\
\hline 650 & 0 & $0.0420 \pm 0.0048 \pm 0.0034$ \\
\hline 700 & 0 & $0.0196 \pm 0.0028 \pm 0.0017$ \\
\hline 750 & 0 & $0.01049 \pm 0.00188 \pm 0.00092$ \\
\hline 800 & 0 & $0.00430 \pm 0.00112 \pm 0.00038$ \\
\hline 850 & 0 & $0.0019 \pm 0.00072 \pm 0.00016$ \\
\hline 900 & 0 & $0.000807 \pm 0.000464 \pm 0.000071$ \\
\hline 950 & 0 & $0.000538 \pm 0.000377 \pm 0.000047$ \\
\hline 1000 & 0 & $0.000269 \pm 0.000266 \pm 0.000024$ \\
\hline
\end{tabular}

Table C.3: Number of events above mass for the ECCC topology. 


\begin{tabular}{|cccc|}
\hline $\begin{array}{c}\text { Mass } \\
(\mathrm{GeV})\end{array}$ & $\begin{array}{c}\text { Lower } \\
\text { Mass Bound } \\
(\mathrm{GeV})\end{array}$ & $\begin{array}{c}\text { Data } \\
\text { Events }\end{array}$ & $\begin{array}{c}\text { Expected Background } \\
\text { Events }\end{array}$ \\
\hline \hline 400 & 342.1 & 20 & $21.3 \pm 2.3$ \\
500 & 431 & 7 & $7.91 \pm 0.82$ \\
600 & 520.2 & 3 & $2.62 \pm 0.28$ \\
650 & 562.1 & 3 & $1.78 \pm 0.19$ \\
700 & 604.9 & 2 & $1.23 \pm 0.13$ \\
750 & 645.6 & 1 & $0.919 \pm 0.093$ \\
800 & 689.9 & 0 & $0.680 \pm 0.067$ \\
850 & 734.5 & 0 & $0.541 \pm 0.052$ \\
900 & 778.2 & 0 & $0.46 \pm 0.044$ \\
1000 & 857.8 & 0 & $0.0482 \pm 0.0069$ \\
\hline
\end{tabular}

Table C.4: Number of events in asymmetric mass window for both electrons in CCCC fiducial sub-sample.

\begin{tabular}{|cccc|}
\hline $\begin{array}{c}\text { Mass } \\
(\mathrm{GeV})\end{array}$ & $\begin{array}{c}\text { Lower } \\
\text { Mass Bound } \\
(\mathrm{GeV})\end{array}$ & $\begin{array}{c}\text { Data } \\
\text { Events }\end{array}$ & $\begin{array}{c}\text { Expected Background } \\
\text { Events }\end{array}$ \\
\hline \hline 400 & 342.1 & 4 & $5.3 \pm 0.56$ \\
500 & 431 & 1 & $1.30 \pm 0.14$ \\
600 & 520.2 & 1 & $0.471 \pm 0.053$ \\
650 & 562.1 & 1 & $0.294 \pm 0.033$ \\
700 & 604.9 & 1 & $0.180 \pm 0.021$ \\
750 & 645.6 & 1 & $0.112 \pm 0.013$ \\
800 & 689.9 & 1 & $0.0654 \pm 0.0082$ \\
850 & 734.5 & 0 & $0.0394 \pm 0.0053$ \\
900 & 778.2 & 0 & $0.0234 \pm 0.0035$ \\
1000 & 857.8 & 0 & $0.00879 \pm 0.0017$ \\
\hline
\end{tabular}

Table C.5: Number of events in asymmetric mass window for one electron in CCCC fiducial sub-sample. 


\begin{tabular}{|cccc|}
\hline $\begin{array}{c}\text { Mass } \\
(\mathrm{GeV})\end{array}$ & $\begin{array}{c}\text { Geometric } \\
\text { Acceptance }\end{array}$ & $\begin{array}{c}\text { Window } \\
\text { Efficiency }\end{array}$ & $\begin{array}{c}\text { Total } \\
\text { Acceptance }\end{array}$ \\
\hline \hline 400 & $0.4957 \pm 0.0054$ & $0.607 \pm 0.025$ & $0.301 \pm 0.012$ \\
500 & $0.5253 \pm 0.0054$ & $0.609 \pm 0.027$ & $0.320 \pm 0.012$ \\
600 & $0.5478 \pm 0.0056$ & $0.635 \pm 0.031$ & $0.348 \pm 0.014$ \\
650 & $0.5655 \pm 0.0055$ & $0.643 \pm 0.031$ & $0.364 \pm 0.014$ \\
700 & $0.5749 \pm 0.0054$ & $0.645 \pm 0.033$ & $0.371 \pm 0.015$ \\
750 & $0.5743 \pm 0.0057$ & $0.646 \pm 0.035$ & $0.371 \pm 0.015$ \\
800 & $0.6170 \pm 0.0057$ & $0.654 \pm 0.037$ & $0.403 \pm 0.018$ \\
850 & $0.6165 \pm 0.0057$ & $0.646 \pm 0.040$ & $0.398 \pm 0.019$ \\
900 & $0.6055 \pm 0.0059$ & $0.666 \pm 0.044$ & $0.403 \pm 0.021$ \\
1000 & $0.6434 \pm 0.0068$ & $0.667 \pm 0.057$ & $0.429 \pm 0.035$ \\
\hline
\end{tabular}

Table C.6: Z' signal acceptance for both in fiducial in CCCC topology.

\begin{tabular}{|cccc|}
\hline $\begin{array}{c}\text { Mass } \\
(\mathrm{GeV})\end{array}$ & $\begin{array}{c}\text { Geometric } \\
\text { Acceptance }\end{array}$ & $\begin{array}{c}\text { Window } \\
\text { Efficiency }\end{array}$ & $\begin{array}{c}\text { Total } \\
\text { Acceptance }\end{array}$ \\
\hline \hline 400 & $0.4957 \pm 0.0054$ & $0.245 \pm 0.015$ & $0.1212 \pm 0.0056$ \\
500 & $0.5253 \pm 0.0054$ & $0.223 \pm 0.014$ & $0.1170 \pm 0.0054$ \\
600 & $0.5478 \pm 0.0056$ & $0.223 \pm 0.014$ & $0.1219 \pm 0.0057$ \\
650 & $0.5655 \pm 0.0055$ & $0.211 \pm 0.017$ & $0.1196 \pm 0.0056$ \\
700 & $0.5749 \pm 0.0054$ & $0.209 \pm 0.016$ & $0.1203 \pm 0.0057$ \\
750 & $0.5743 \pm 0.0057$ & $0.213 \pm 0.013$ & $0.1224 \pm 0.0059$ \\
800 & $0.6170 \pm 0.0057$ & $0.208 \pm 0.017$ & $0.1286 \pm 0.0066$ \\
850 & $0.6165 \pm 0.0057$ & $0.209 \pm 0.016$ & $0.1288 \pm 0.0069$ \\
900 & $0.6055 \pm 0.0059$ & $0.202 \pm 0.018$ & $0.1222 \pm 0.0072$ \\
1000 & $0.6434 \pm 0.0068$ & $0.187 \pm 0.019$ & $0.120 \pm 0.011$ \\
\hline
\end{tabular}

Table C.7: Z' signal acceptance for only one electron in fiducial in CCCC topology. 